NASA

Technical

Paper

3600

ATCOM

Technical

Report

95-A-005

February 1996

Suppression of Dynamic Stall by

Steady and Pulsed Upper-Surface

Blowing

D. Weaver, K. W. McAlister, and J. Tso

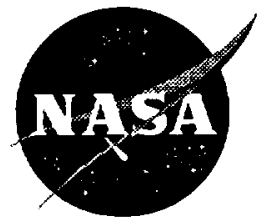

National Aeronautics and Space Administration

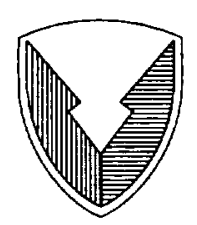

US Army

Aviation and Troop Command

Aeroflightdynamics Directorate Moffett Field, CA 94035-1000 


\section{$+\infty$}

vat

$+$

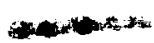


NASA

Technical

Paper

3600

\section{ATCOM \\ Technical \\ Report \\ 95-A-005}

1996

\section{Suppression of Dynamic Stall by Steady and Pulsed Upper-Surface Blowing}

D. Weaver

California Polytechnic State University, San Luis Obispo, California

K. W. McAlister

U.S. Army Aeroflightdynamics Directorate, USAAVRDEC-ATCOM, Ames Research Center, Moffett Field, California

and

J. Tso

California Polytechnic State University, San Luis Obispo, California

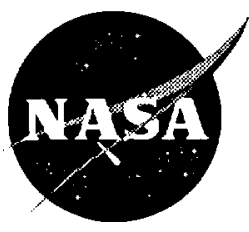

National Aeronautics and Space Administration

Ames Research Center

Moffett Field, California 94035-1000

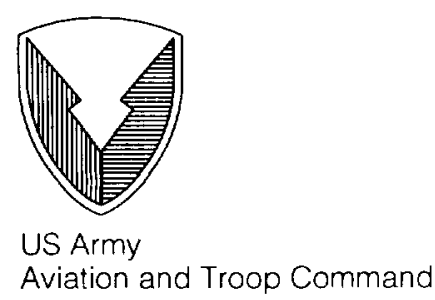

Aeroflightdynamics Directorate Moffett Field, CA 94035-1000 



\section{CONTENTS}

Page

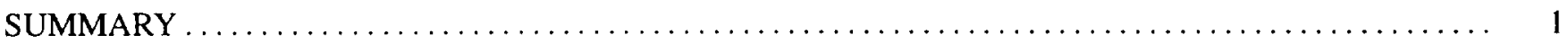

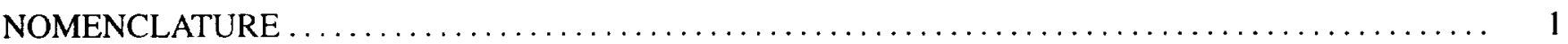

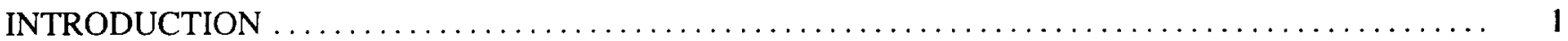

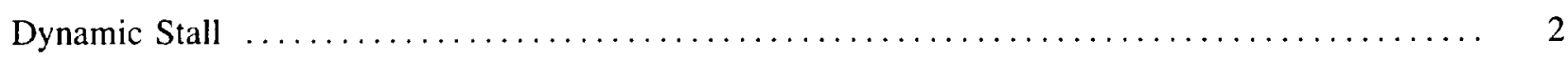

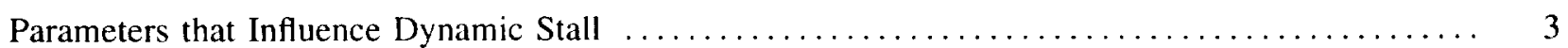

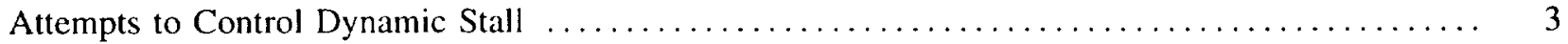

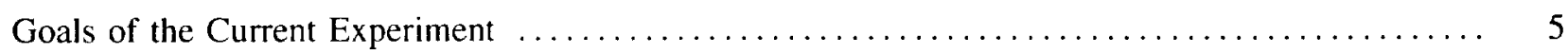

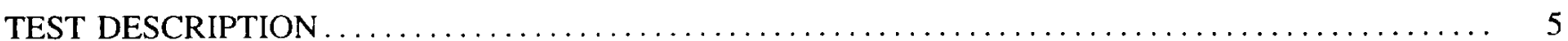

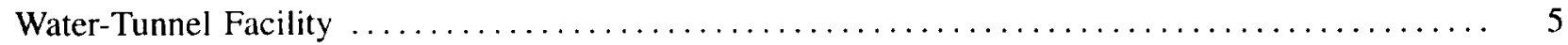

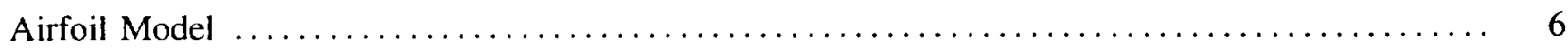

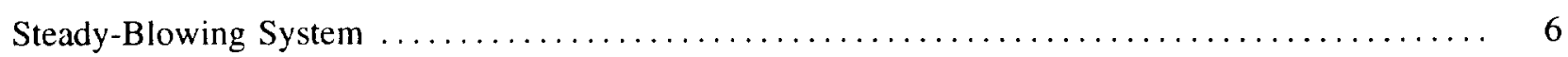

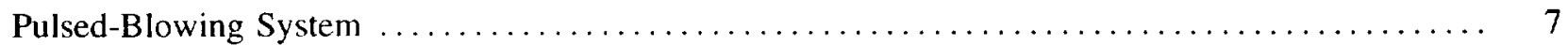

Airfoil Installation and Instrumentation $\ldots \ldots \ldots \ldots \ldots \ldots \ldots \ldots \ldots \ldots \ldots \ldots \ldots \ldots \ldots \ldots \ldots \ldots \ldots \ldots$

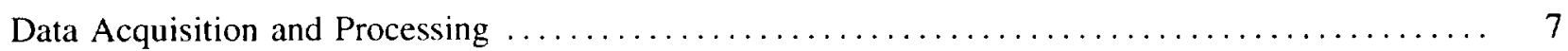

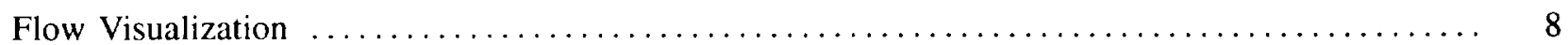

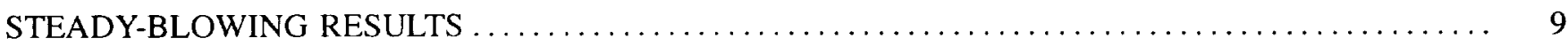

Steady Blowing with $\alpha_{m}=10^{\circ}$ and $k=0.005$ (nominal) $\ldots \ldots \ldots \ldots \ldots \ldots \ldots \ldots \ldots \ldots$

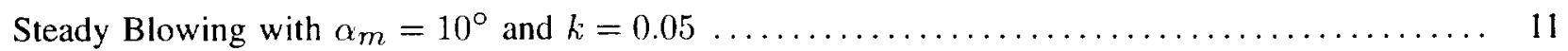

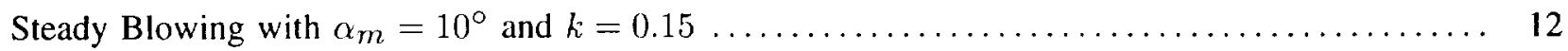

Steady Blowing with $\alpha_{m}=15^{\circ}$ and $k=0.007$ (nominal) $\ldots \ldots \ldots \ldots \ldots \ldots \ldots \ldots \ldots \ldots \ldots$

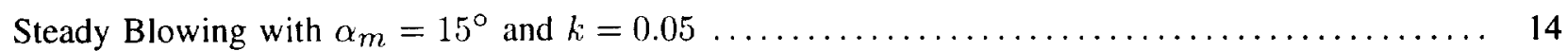

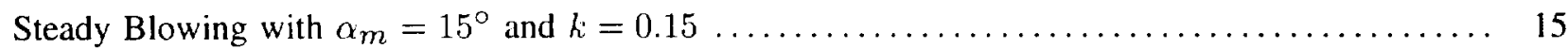

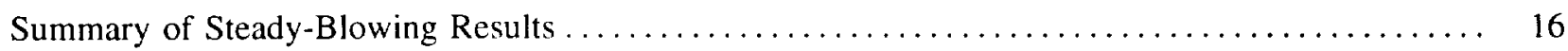

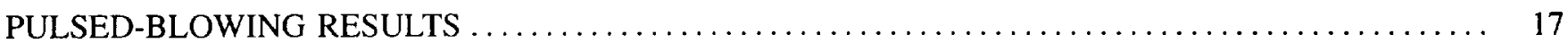

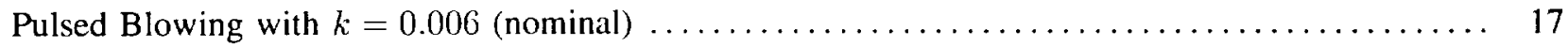

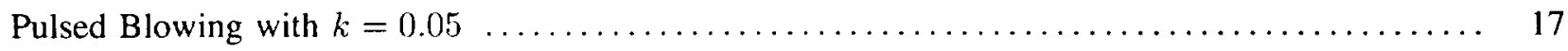

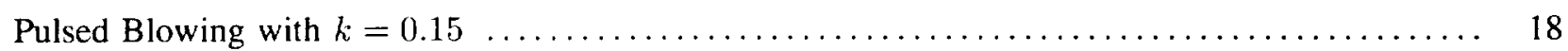

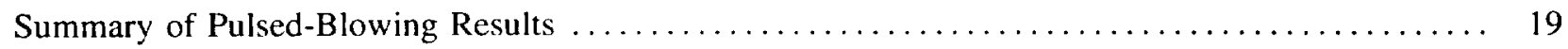

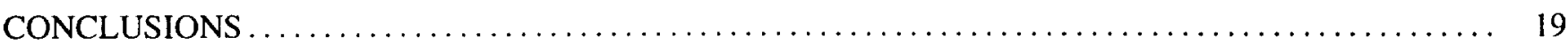

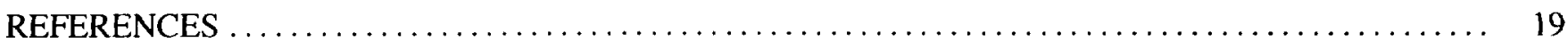

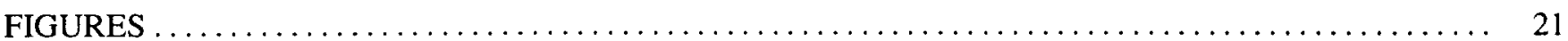





\title{
Suppression of Dynamic Stall by Steady and Pulsed Upper-Surface Blowing
}

\author{
D. Weaver, ${ }^{*}$ K. W. McAlister, ${ }^{\dagger}$ and J. Tso* \\ Ames Research Center
}

\section{SUMMARY}

The Boeing-Vertol VR-7 airfoil was experimentally studied with steady and pulsed upper-surface blowing for sinusoidal pitching oscillations described by $\alpha=\alpha_{m}+10^{\circ} \sin \omega t$. The tests were conducted in the U.S. Army Aeroflightdynamics Directorate's Water Tunnel at NASA Ames Research Center. The experiment was performed at a Reynolds number of 100,000. Pitch oscillations with $\alpha_{m}=10^{\circ}$ and $15^{\circ}$ and with reduced frequencies ranging from $k=0.005$ to 0.15 were examined. Blowing conditions ranged from $C_{\mu}=0.03$ to 0.66 and $F^{+}=0$ to 3 . Unsteady lift, drag, and pitching-moment loads were measured, and fluorescent-dye flow visualizations were obtained. Steady, upper-surface blowing was found to be capable of trapping a separation bubble near the leading edge during a portion of the airfoil's upward rotation. When this occurred, the lift was increased significantly and stall was averted. In all cases, steady blowing reduced the hysteresis amplitudes present in the loads and produced a large thrust force. The benefits of steady blowing diminished as the reduced frequency and mean angle of oscillation increased. Pulsed blowing showed only marginal benefits for the conditions tested. The greatest gains from pulsed blowing were achieved at $F^{+}=0.9$.

\section{NOMENCLATURE}

$\begin{array}{ll}b & \text { model span } \\ c & \text { model chord } \\ C_{d} & \text { drag coefficient, } \mathrm{drag} /\left(q_{\infty} S\right) \\ C_{l} & \text { lift coefficient, lift } /\left(q_{\infty} S\right) \\ C_{m} & \text { moment coefficient, moment } /\left(q_{\infty} S c\right)\end{array}$

*California Polytechnic State University, San Luis Obispo, California.

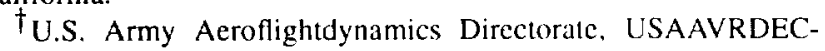
ATCOM, Ames Research Center, Moffett Ficld, California.

$\begin{array}{ll}C_{\mu} & \text { steady-blowing coefficient, } \\ & J /\left(q_{\infty} c\right)=2(h / c)\left(V_{s} / V_{\infty}\right)^{2} \\ f & \text { pulsing frequency } \\ F^{+} & \text {dimensionless pulsing frequency, } \\ & f x_{t c} / V_{\infty} \\ h & \text { slot height } \\ J & \text { mean momentum at slot exit (per unit } \\ & \quad \text { span), } \rho V_{s}^{2} h \\ k & \text { reduced frequency, } \omega c /\left(2 V_{\infty}\right) \\ Q_{s} & \text { volume-flow rate through slot } \\ q_{\infty} & \text { dynamic pressure, } \frac{1}{2} \rho V_{\infty}^{2} \\ R e & \text { Reynolds number, } c V_{\infty} / \nu \\ S & \text { planform area of airfoil, }(b \times c) \\ t & \text { time } \\ V_{S} & \text { mean slot-exit velocity } \\ V_{\infty} & \text { free-stream velocity } \\ x & \text { distance along chord from leading edge } \\ x_{t e} & \text { distance from slot to trailing edge } \\ \alpha & \text { angle of attack } \\ \alpha & \text { mean angle of oscillation } \\ \zeta & \text { pitch damping } \\ \nu & \text { fluid kinematic viscosity } \\ \rho & \text { fluid density } \\ \omega & \text { frequency of pitch oscillation }\end{array}$

\section{Acronyms}

AFDD U.S. Army Aeroflightdynamics Directorate

LDV Laser Doppler Velocimetry

LED Light-emitting diodes

\section{INTRODUCTION}

The helicopter is one of the most versatile aircraft in operation today. It can take off and land with a substantial payload from virtually any area having sufficient clearance for the rotor and tail boom. It also has the unique ability to hover efficiently, allowing it to remain on station for long periods of time while performing rescue operations, payload delivery, or on 
many other civilian and military functions. This versatility ensures that the helicopter will continue to be a valuable asset to our nation's well-being and security, so it is prudent to continuously improve the performance of this unique aircraft. The most significant barrier to higher performance is a phenomenon called retreating-blade stall (ref. 1). Retreating-blade stall results from the unique aerodynamic environment of the main rotor. To understand the occurrence, two issues must be considered. First, in a forward-flight situation, the rotor blades experience a sinusoidal change in angle of attack. This cyclic change is required to balance the lift forces between the advancing and retreating sides of the rotor (ref. 2). The pitch angle of the advancing blade decreases while the pitch angle of the retreating blade increases. Second, to reach higher forward speeds, the rotor must produce more net thrust, which is accomplished by increasing the pitch on all the blades collectively. Eventually, as the helicopter approaches the edge of its speed envelope, any further increases in pitch will lead to stall on the retreating side of the rotor. When this occurs, an asymmetric lift condition results and causes a rolling moment that could lead to a catastrophe. In addition, severe rotor vibration is experienced because of a stall event that is unique to a wing experiencing a rapid increase in angle of attack (ref. 3). Thus, to expand the performance envelope of the helicopter, researchers must understand the mechanics that underlie the stall behavior of the rotor blade.

\section{Dynamic Stall}

Retreating-blade stall is an example of an event called dynamic stall. Dynamic stall is the process of boundary-layer separation from an airfoil or wing experiencing a dynamic increase in angle of attack (refs. 1, 4 , and 5). In the case of retreating-blade stall, the increase in angle of attack is due to the sinusoidalpitching motion that is experienced by helicopter rotor blades. Past research shows that this process, like the stalls on airfoils at fixed angles of attack, can be investigated with simple, two-dimensional experiments because the rotational effects on the flow field are considered small (refs. 1, 4-6). Additionally, since rotor blades typically have a high aspect ratio, tip effects are also of secondary importance. To date, several experiments have been conducted on oscillating airfoils with a sinusoidal-pitching motion defined by

$$
\alpha=\alpha_{m}+10^{\circ} \sin \omega t
$$

where $\alpha$ is the angle of attack, $\alpha_{m}$ is the mean angle of attack, $\omega$ is the frequency of pitch oscillation, and $t$ is time. These experiments have shown that dynamic stall is quite different from its quasi-steady counterpart.

The differences between dynamic stall and quasisteady stall stem from the airfoil's motion $(d \alpha / d t)$. Three prominent differences can be identified. The first difference is the appearance of a large amount of load hysteresis with respect to angle of attack in the unsteady case (refs. 1, 4, and 5). Because the airfoil is oscillating, the relative flow velocity experienced by the airfoil is different during each half of the cycle. In particular, the boundary layer is attached for nearly all of the "upstroke," but it is separated for most of the "downstroke."

The second difference that is attributable to the airfoil's motion is a phenomenon called "lift overshoot" (refs. 1, 4, and 5). Past experiments show that a pitching airfoil tolerates large regions of reversed flow on its surface before experiencing large-scale, boundarylayer separation (ref. 4). This tolerance allows the airfoil to rotate well beyond the quasi-steady stall angle and produces a much higher lift force than it would normally be capable of. The physical explanation typically given for the delay in full-scale separation is that a finite time period is necessary for the flow in the boundary layer to react to the pitching motion. Additionally, theoretical studies of laminar boundary layers show that the boundary-layer equations have a singularity associated with the point of flow separation for steady flow. This singularity is distinctly different from the point of flow reversal for an unsteady flow, thus allowing the existence of flow reversal without full-scale separation (ref. 4).

The third difference between dynamic stall and quasi-steady stall is the shedding of intense vorticity from the upper surface (refs. 1, 4, and 5). The concentrated vorticity that is generated and then shed from the airfoil is commonly referred to as the dynamic-stall vortex. This shedding event, which normally occurs during the upstroke, causes impulsive changes in the loads on the airfoil and is accompanied by large-scale, boundary-layer separation.

An example of dynamic stall on a VR-7 airfoil is illustrated in figure 1. The example shows that the airfoil overshoots the quasi-steady stall angle and generates more lift than it normally would in a quasi-steady environment. As the incidence continues to increase, a 
thin layer of reversed flow appears at the bottom of the boundary layer (ref. 4). Note that no significant distortions of the external stream are present other than a small flow protuberance at the leading edge. This flow protuberance marks the initial formation of the dynamic-stall vortex. The dynamic-stall vortex, like a laminar-separation bubble, is a region of high vorticity that lowers the pressure at the leading edge, increases the lift slope, and creates a positive distortion in the pitching moment (ref. 4).

The vortex grows as the airfoil continues to rotate upward, and it eventually begins to migrate toward the trailing edge at a speed of roughly $V_{\infty} / 2$ (ref. 5). This event is referred to as "moment stall" because the motion of the vortex over the surface shifts the center of pressure and induces a large, "nose-down," pitching moment (ref. 4). Large eddies also form in the boundary layer by the time moment stall begins. As the vortex moves over the surface, the large eddies in the boundary layer coalesce into one unified structure that is shed from the trailing edge. Maximum lift occurs as the vortex passes between the quarter-chord and mid-chord points. This is followed by a sharp collapse of the lift force (called "lift stall"). As the vortex approaches the trailing edge, the moment coefficient reaches its largest magnitude.

The airfoil is fully stalled when the vortex leaves the trailing edge. Reestablishment of the boundary layer occurs during the downstroke of the airfoil's oscillation. This process occurs slowly and is usually not complete until the downstroke is almost finished. When the attached boundary layer is completely reestablished, the forces and moments return to their former, approximately linear, behavior.

\section{Parameters that Influence Dynamic Stall}

Many factors have been found to influence the process of dynamic stall. Light stall, characterized by a relatively weak stall vortex and a viscous zone on the order of the airfoil thickness, is found to be sensitive to the same geometrical parameters that affect quasisteady stall (refs. 1, 4, and 5). Leading-edge radius and airfoil camber are two examples of parameters that can affect light stall. Light stall typically occurs for low oscillation rates and for oscillation amplitudes that do not allow the airfoil to venture very far past the quasisteady stall angle. Deep stall, on the other hand, is relatively unaffected by these considerations (refs. 1, 4 , and 5).
Deep stall exhibits large-scale vortex shedding as its predominant feature. Large force overshoots and hysteresis amplitudes are present, and the scale of the viscous zone is on the order of the airfoil chord length. Deep stall is heavily influenced by the amount of time the airfoil spends exceeding the quasi-steady stall angle. Deep stall usually occurs during rapid-pitch oscillations that have a combination of mean angle and amplitude values that allow the airfoil to spend a large percentage of time beyond the quasi-steady stall angle.

A non-dimensional parameter that is used to quantify the speed of an airfoil's oscillation is the "reduced frequency." The reduced frequency is defined by

$$
k=\frac{\omega c}{2 V_{\infty}}
$$

where $c$ is the model chord and $V_{\infty}$ is the free-stream velocity. Physically, the reduced frequency represents the portion of the oscillation cycle, in radians, that elapses during the time it takes the free-stream flow to travel half a chord length (ref. 2). During oscillations with a high reduced frequency, the dynamic-stall vortex is often shed late in the upstroke or at some point early in the downstroke (refs. 1, 4, and 5). After the vortex is shed, boundary-layer separation usually persists for most of the downstroke because of the rapid oscillation rate. Large hysteresis amplitudes that are common to deep stall result.

\section{Attempts to Control Dynamic Stall}

The discovery that profile shape had little or no effect on the nature of a deep, dynamic stall motivated researchers to look for other ways to control the event. Previous work done by the U.S. Army Aeroflightdynamics Directorate (AFDD), located at the NASA Ames Research Center, yielded several promising methods. The approaches that gave positive results fell into three main categories: leading-edge slats, deformable airfoils, and upper-surface blowing (ref. 7). The methods were evaluated using both numerical and laboratory techniques. A brief summary of previous work is provided in figure 2 .

Slatted airfoils- The use of a leading-edge slat was one of the most recent attempts made by the AFDD to control dynamic stall. Two Boeing-Vertol airfoils, the VR-7 (ref. 8) and VR-12 (ref. 9), were fitted with different styles of leading-edge slats and tested in the Army's closed-circuit water tunnel. The best results 
were obtained with the VR-7 airfoil fitted with an optimally positioned NACA 15320 slat (ref. 8). The slat eliminated the formation of the dynamic-stall vortex for reduced frequencies up to 0.2 and for oscillations as high as $20^{\circ}+10^{\circ} \sin \omega t$. As a result, the severe, nosedown, pitching moment that normally accompanies the migration of the stall vortex was averted. Hysteresis amplitudes and the peak values for drag and pitching moment were also dramatically decreased because of a reduction of boundary-layer separation. The only negative effect observed was an increase in the drag coefficient at low angles of attack.

The VR-12 airfoil was configured with a slat by simply extending a portion of the leading edge forward and contouring the lower surface (ref. 9). Unlike the previous attempt, the results indicated that the simple extendible slat did not eliminate dynamic stall on the VR-12. At high reduced frequencies and mean angles of attack, vortex migration was still observed. A good deal of flow separation also prevented any marked reduction in the hysteresis amplitudes, although some improvement was observed because of an earlier reestablishment of the boundary layer. An increase in the drag coefficient at low angles of attack was also observed on the slatted VR-12.

Deformed airfoils- Another effective attempt at controlling dynamic stall was the use of a deformed leading edge (ref. 10). A VR-12 airfoil was deformed by drooping the forward $25 \%$ by $13^{\circ}$. It was hypothesized that the deformed shape enabled the flow to pass smoothly over the area where the dynamic-stall vortex normally forms. The results of water tunnel testing indicated that the dynamic-stall vortex did not form and that the hysteresis amplitudes were significantly reduced. The maximum pitching moment and drag coefficients were much lower than those of the unmodified airfoil. As with the airfoils with slats, the deformed airfoil experienced a drag penalty at lower angles of attack because of the profile change.

Steady upper-surface blowing- One of the AFDD's earliest attempts to control dynamic stall involved the use of upper-surface blowing. This method increases the momentum of the flow in the boundary layer by injecting mass from a slot on the upper surface. The increased momentum allows the boundary layer to push through the region of adverse pressure gradient without separating from the surface. This method does not incur a drag penalty at low angles of attack because it does not change the profile shape of the airfoil. The slot momentum coefficient, an important parameter, defines the relative strength of blowing. It is defined as

$$
C_{\mu}=\frac{J}{q_{\infty} c}=2\left(\frac{h}{c}\right)\left(\frac{V_{s}}{V_{\infty}}\right)^{2}
$$

where $h$ is the slot height, and $V_{s}$ the mean slot-exit velocity. Physically, this coefficient represents the ratio of slot momentum to free-stream momentum. The momentum coefficient can also be interpreted as the force coefficient per unit span along the axis of blowing. This relationship is easily obtained by regrouping terms in equation (3) as follows

$$
C_{\mu}=\frac{J}{q_{\infty} c}=\frac{\rho(h b) V_{s}^{2} / b}{q_{\infty} c}
$$

where $b$ is the model span and $q_{\infty}$ is the free-stream dynamic pressure. The numerator represents the thrust per unit span contributed by blowing. The denominator serves to form a non-dimensional coefficient similar to the lift and drag coefficients. To further amplify the physical meaning of this quantity, if the blowing jet is located at the trailing edge of the airfoil and is pointed in the free-stream direction, then the drag coefficient would be decreased by an amount equal to $C_{\mu}$.

During the early 1980s, an informal flowvisualization study of this technique was conducted in the AFDD Water Tunnel Facility (ref. 7). A NACA 0012 airfoil was fitted with a blowing slot on the quarter-chord line of the upper surface. Pressurized water was fed into the airfoil's boundary layer from the slot. The results demonstrated that blowing was insufficient to prevent the dynamic-stall vortex from forming, but that it was capable of trapping the dynamicstall vortex near the leading edge. With the vortex trapped on the upper surface, it was hypothesized that an extraordinarily high-lift force could be achieved and the severe load fluctuations caused by a migrating vortex could be eliminated. Unfortunately, the water tunnel facility was not equipped to measure loads at the time of the experiment, so the precise effect of blowing on the lift, drag, and pitching moment could not be determined. However, the visualization photographs presented clear evidence that the highest blowing rate was most effective.

Pulsed upper-surface blowing- A method of blowing that has shown some promise on airfoils at fixed angles of attack is pulsed blowing. Pulsed blowing, in contrast to steady blowing, sends short pulses 
of fluid into the boundary layer rather than a continuous jet. The pulses are believed to produce vorticity within the boundary layer. The vorticity transports momentum to the boundary layer from the free-stream flow. By using vorticity to transport momentum from the free-stream, less momentum is required from the jet itself. This method appears to have been successful on static airfoils, and researchers believed that the same principle may be useful in controlling dynamicstall behavior. In a pulsed-blowing situation, the $V_{s}$ term appearing in the expression for the momentum coefficient (eq. (3)) should be interpreted as the rootmean-square value of the jet velocity at the slot exit.

One attempt to control stall on a static airfoil using pulsed blowing was made by researchers from Tel Aviv University (refs. 11 and 12). They conducted pulsed-blowing experiments on a NACA 0015 airfoil equipped with a blowing slot at the hinge of a trailingedge flap (ref. 11) and a NACA 0015 airfoil equipped with a blowing slot at the leading edge (ref. 12). The results were presented for a Reynolds number range of 150,000 to 750,000 . They discovered that pure pulsed blowing from the leading edge at a very low momentum coefficient, $C_{\mu}$, of 0.0008 and at a dimensionless pulsing frequency, $F^{+}$, near 0.8 increased the lift coefficient by $30 \%$ (relative to the case with no blowing) at $\alpha=16^{\circ}$. The dimensionless pulsing frequency is defined by

$$
F^{+}=\frac{f x_{t e}}{V_{\infty}}
$$

where $f$ is the pulsing frequency and $x_{t e}$ is the distance from the slot to the trailing edge.

This is a significant accomplishment in view of the fact that steady blowing at the same $C_{\mu}$ hardly produced any effect at all (ref. 12). Generally, they concluded that pulsed blowing worked best when there were one or two pulse disturbances on the surface at any given time. They also concluded that the preferred range of dimensionless pulsing frequency, $0.3 \leq F^{+} \leq 1.0$, was a function of Reynolds number and angle of attack prior to stall, but it became independent of these two parameters in the post-stall region.

Another attempt to control stall on an airfoil at fixed angles using pulsed blowing was made by researchers from Washington State University (ref. 13). These investigators also conducted their experiments on the NACA 0015 airfoil. Pulsed blowing was accomplished by blowing compressed air through three slots on the forward half of the upper surface. A $20 \%$ increase in the lift coefficient (relative to the case with no blowing) was achieved at $16^{\circ}$ for a Reynolds number of 57,000 , a momentum coefficient of 0.01 , and a pulse frequency of $30 \mathrm{~Hz}$. The frequency of the pulsing corresponds to an $\mathrm{F}^{+}$of approximately 0.7 at standard atmospheric conditions, and it is consistent with the results of reference 12 . They also concluded that the preferred frequency of pulsing was a function of Reynolds number and angle of attack prior to stall, but the frequency of pulsing became independent of these two parameters in the post stall region.

\section{Goals of the Current Experiment}

This study was performed to quantify the effects of steady, upper-surface blowing on an airfoil's dynamic-stall behavior and to explore the possible benefits of pulsed blowing in a dynamic-stall environment. Unsteady loads were measured on a VR-7 airfoil oscillating about its quarter-chord axis with a sinusoidalpitching motion defined by equation (1). Force and moment data were obtained for the basic airfoil and for both types of upper-surface blowing. Measurements were made at a Reynolds number of 100,000 , where Reynolds number is defined by

$$
R e=\frac{c V_{\infty}}{\nu}
$$

The data matrix (nominal values) included mean angles, $\alpha_{m}$, of $10^{\circ}$ and $15^{\circ}$; blowing coefficients, $C_{\mu}$, between 0.03 and 0.66 ; pulsing frequencies, $F^{+}$, between 0 and 3 ; and reduced frequencies, $k$, of 0.005 , 0.05 , and 0.15 . Flow-visualization photographs were taken for each cycle to compliment the force and moment data.

\section{TEST DESCRIPTION}

\section{Water-Tunnel Facility}

The test was conducted in the U.S. Army Aeroflightdynamics Directorate (AFDD) closed-circuit water tunnel at the NASA Ames Research Center. The facility offers several advantages for testing an airfoil in an unsteady-flow environment. The most significant advantage is the facility's instrumentation. It is designed specifically for measuring the loads on an oscillating airfoil. Another advantage is the ability to obtain 
high quality flow visualization. The flow-visualization equipment has been refined over the years and has produced some of the finest visualizations in the field. A photograph of the facility is provided in figure 3 .

The tunnel's test section measures 34 in. long (chordwise direction), 8.3 in. wide (spanwise direction), and 12 in. high (ref. 8). All four walls of the rectangular test section are composed of removable plexiglas windows set into a stainless-steel frame. The windows allow models to be viewed from several directions. Models are mounted with their spars protruding through openings in the sidewalls of the test section. The ends of the spar are supported on both sides of the test section by load cells in the lift and drag directions. Torsionally stiff bellows and lip seals provide water containment. Figure 4 shows the mounting arrangement. A honeycomb and several screens are positioned upstream of the test section in the settling chamber to straighten the flow. These flowstraightening devices also reduce the turbulence intensity to about $0.05 \%$ in the test section when there is no model present (ref. 8). With a stalled model in the test section, the turbulence intensity can increase by a factor of five (ref. 8).

\section{Airfoil Model}

The model used for the test was a Boeing-Vertol VR-7 airfoil with a negative $3^{\circ}$ trailing-edge tab (an upward deflection of the trailing edge). The airfoil's profile is shown in figure 5. Since the goal of the experiment was to investigate the effects of upper-surface blowing, the design of the model was a formidable task. It was necessary to incorporate a slot on the upper surface and to provide internal plumbing to supply the slot in a uniform manner.

The internal design of the model was governed by several considerations. When a model is installed in the test section, the oscillatory-drive mechanism connects to the spar of the model on the starboard side. This makes it impractical to supply water from both ends of the model. Experience with two previous slotted models demonstrated that when water was supplied to an internal plenum (a simple cavity without flow controls) from one end of the model only, a nonuniform slo: velocity resulted across the span. The velocity was always much higher at the end of the slot opposite the supply. For this reason, a diffuser was chosen to supply the slot rather than a plenum. It was hypothesized that fanning the internal flow out from a central location within the model would result in a better spanwise velocity distribution across the slot.

To provide an internal diffuser, the model was assembled from five pieces. The construction is shown in figures 6 and 7. The main body of the airfoil consists of a base plate that forms the entire lower surface. The upper surface is composed of two pieces fastened to the base plate. Two end plates are fastened to the main body of the model to provide spar shafts. Fully assembled, the model has a chord length of 4 in., and it spans the test section. It is constructed entirely of stainless steel to provide durability in a water environment.

A contoured gap between the two upper-surface pieces, located at the quarter chord, serves as the blowing slot. The dimensions of the slot are given in table 1. Several diffuser passages are cut into the interior of the upper-rear piece to deliver flow to the slot. Each passage has a similar area ratio, and they all fan out from a central location. The diffuser is fed through a passage inside the spar (and coincident with the quarter-chord axis) from the port side of the model.

Table 1. Airfoil and slot dimensions

\begin{tabular}{ll}
\hline Slot height, $\mathrm{h}$ & $0.003 \mathrm{in.}$ \\
Airfoil chord, $\mathrm{c}$ & $4.0 \mathrm{in.}$ \\
Slot span, $\mathrm{b}$ & $7.9 \mathrm{in}$. \\
\hline
\end{tabular}

To perform flow visualization, two dye ports were installed on the model. Both ports are located at center span. One port is located at the leading edge and the other is located within the slot. The ports are fed by separate passages that run through the interior of the model. The passages are connected to separate tubes that enter the model from the starboard side.

\section{Steady-Blowing System}

To perform the steady-blowing experiments, flow to the slot on the upper surface of the model was provided by a flexible hose attached to the model's spar (port side). The hose was connected to two pumps (operated in series) that pulled water from the tunnel. This arrangement provided water to the slot with the same temperature as the free-stream flow. The use of two pumps was required to supply sufficient pressure for a wide range of blowing momentum coefficient, $C_{\mu}$. 
A globe valve and a venturi-style flow meter were located between the two pumps to accurately control and monitor the volume-flow rate through the slot. Figure 8 shows a schematic of the blowing system.

\section{Pulsed-Blowing System}

To perform pulsed blowing, a rotary valve was placed in the line between the wing and the venturistyle flow meter. The valve operated much like an old west "six-shooter." Water entered a holding chamber, and pulses of water were released when holes in a rotating plate aligned with an exit hole in the valve body. By pulsing the water in this manner, an on-off blowing situation was created. In other words, the valve caused the mass-flow rate to oscillate between zero and some maximum value. The valve was constructed of anodized aluminum to provide corrosion resistance. A V-belt connected to a $3 / 4-\mathrm{hp}$-dc motor was used to drive the valve's rotor. The rotational speed of the motor was adjusted with an electronic speed controller. There was no particular phase relationship between the frequency of the blowing pulses, $f$, and the frequency of airfoil oscillation, $\omega$, since $f \gg \omega$. For example, when $F^{+}=0.9$ and $k=0.15$, then $f=12 \mathrm{~Hz}$ and $\omega / 2 \pi=0.5 \mathrm{~Hz}$.

\section{Airfoil Installation and Instrumentation}

The water-tunnel facility was equipped with a suspension system designed to measure unsteady hydrodynamic loads on oscillating test models. The model suspension system is shown in figure 4. Models are mounted with their spars protruding through openings in the sidewalls of the test section. On both sides of the test section, the ends of the spar pass through ball bearings that are supported by load cells in the lift and drag directions. The ball bearings allow the model to rotate freely about an axis passing through the spar and along the quarter-chord line of the airfoil.

Torsionally-stiff bellows and lip seals provide water containment. The bellows, which are flexible in the lateral directions, do not contribute any supporting force to the model; that is, they are stiff in torsion only, providing little or no resistance to bending. This allows the load cells to measure true lift and drag forces directly. Torsional resistance is provided by a mechanical link to a flywheel and motor assembly. This assembly also imparts the pitching motion to the model. Three strain-gauge bridges, one on each of the bellows and one on the mechanical link to the flywheel, measure the pitching-moment characteristics of the model. The bridge on the mechanical link measures the total pitching moment on the model. Two bridges on the bellows measure frictional moments imparted by the support bearings and seals. These are treated as dynamic-load tares and subtracted from the total moment measured at the mechanical link to obtain the pitching moment caused by the flow.

Other instrumentation included a transducer for measuring the airfoil's incidence. A pressure transducer, connected between the settling chamber and the test section, was used to measure the dynamic pressure, from which the free-stream velocity, $V_{\infty}$, of the tunnel was calculated. During the pulsed-blowing portion of the experiment, a stroboscope was used to monitor the rotational speed of the pulse valve.

A challenging problem was created by the addition of the pulse valve. In a pulsed-blowing situation, it is proper to use a root-mean-square slot velocity to compute $C_{\mu}$, since the slot velocity is a periodic function of time. Unfortunately, it would have been a major endeavor to measure the time-varying slot velocity in this facility. To avoid the expense and complexity of a velocity measuring technique like Laser Doppler Velocimetry (LDV), the decision was made to record the time-averaged, volume-flow rate from the venturi-style flow meter and to compute the average, one-dimensional, slot velocity from the principle of volume conservation. Had this experiment involved a purely sinusoidal-blowing rate, this procedure would be unacceptable because the time-averaged rate would have a nominal value of zero. However, in this case the mass-flow rate from the slot was always positive because of the design of the pulse valve. This allowed the time-averaged velocity to be used, rather than the root-mean-square velocity.

Calibration efforts for the instrumentation were minimal. They involved recording the voltage outputs of the instruments in response to known inputs. Calibration curves for the force transducers, strain-gauge bridges, incidence transducer, and pressure transducer were all linear within the ranges of interest. It was estimated that the angle of attack could be measured to an accuracy of $0.2^{\circ}$. Lift and drag measurements were estimated to be accurate to $0.002 \mathrm{lb}$, and pitchingmoment measurements were considered accurate to 0.02 in. - lb. 


\section{Data Acquisition and Processing}

Each of the signals from the measurement devices was amplified, digitized, recorded, and processed by a remote data-acquisition system. The acquisition system was a stand-alone unit consisting of amplifiers, an analog to digital converter, and a Digital VAX 11751 workstation. The acquisition and processing operation was controlled by a FORTRAN program. The program instructed the acquisition system to digitize the data signals based on a $360 /$ rev pulse train that was synchronous with $\omega t$ and a $1 /$ rev pulse that was synchronous with the beginning of each cycle. The program then ensemble averaged the digitized cycles. Data averaging was performed over a period of $30 \mathrm{cy}$ cles for the unsteady data and a period of 5 cycles for the quasi-steady data.

Dynamic-load tares were frequently recorded throughout the experiment to provide current reference (or "zero") levels for all of the transducers. These were obtained by recording data at the "static no-flow" condition (model set at the mean angle of attack with $q_{\infty}=0$ ) and at the "dynamic no-flow" condition (model oscillating slowly with $q_{\infty}=0$ ). Following the averaging operation, each signal was appropriately adjusted using either static or dynamic "zero" references. All of the force and moment data were reduced to engineering coefficients during processing. The engineering coefficients were defined as

$$
\begin{aligned}
C_{l} & =\frac{\text { lift }}{q_{\infty} S} \\
C_{d} & =\frac{d r a g}{q_{\infty} S} \\
C_{m} & =\frac{\text { moment }}{q_{\infty} S c}
\end{aligned}
$$

In these definitions, $q_{\infty}$ is the instantaneous dynamic pressure

$$
q_{\infty}=q_{\infty}(t)
$$

It was necessary to use the instantaneous dynamic pressure because the oscillating airfoil produced a significant variation in tunnel resistance (or blockage) throughout the cycle. At $\alpha=25^{\circ}$, the projected frontal area of the VR-7 airfoil was approximately $14 \%$ of the area of the test section. A variation of $16.5 \%$ in dynamic pressure was observed in the high-amplitude cases. Test parameters, along with plots of each coefficient versus angle of attack, were displayed on a monitor at the facility after each acquisition. If any irregularity had occurred during the process, it could be identified immediately and corrected.

No corrections were applied to the data to account for inertial-pitching moments or to account for forces caused by the injection of water into the airfoil. Corrections were not used for inertial pitching moments because an estimate showed that they were approximately two orders of magnitude less than the fluid-dynamic moments. Corrections were not needed to account for forces caused by the injection of blowing water because the water was injected along the axis of the spar, normal to the lift-drag plane. Additionally, no corrections were used to account for the presence of the tunnel walls.

\section{Flow Visualization}

Flow visualization was performed to help interpret the results of the load measurements. Fluorescent dye was released from two ports located mid span on the model. One port was located at the leading edge of the airfoil, and the other port, inside the blowing slot. The visualization was performed with the force balance removed from the viewing side of the tunnel. This provided an unobstructed view along the axis of the model's spar. Single-frame recordings were made during the oscillation cycles by dimming the lights at the facility, opening the shutter of a camera, and firing a strobe lamp. An electronic monitoring circuit was used to flash the strobe at selectable values of $\omega t$. These values were digitally displayed by light-emitting diodes (LEDs) that were positioned in the field of view of the camera.

The dye used for the visualization was made by mixing a Rhodamine $6 \mathrm{G}$ concentrate with deionized water. This dye has an orange color in ordinary room lighting, but it provides a fluorescent-yellow emission when stimulated by light of a shorter wavelength. The mixture was fed to the model's dye ports by flexible lines running from a pressurized container. When the dye was released from the ports, it flowed downstream into the upper-surface boundary layer and into the wake.

The dye was illuminated by a Xenon strobe that emitted a large quantity of radiation in the ultraviolet range. The strobe cavity had the shape of a long tube so that light from the strobe needed only to be baffled to form a sheet of light with a thickness of about 1 in. The light passed through the upper test-section window and 
straight down onto the airfoil surface. The photographs were taken with a Hasselblad camera loaded with Kodak TMY 6053 black-and-white film (ASA 400).

\section{STEADY-BLOWING RESULTS}

Unsteady loads were measured on the airfoil as it oscillated about its quarter-chord axis for several mean angles and reduced frequencies that represent typical rotor environments. The oscillation amplitude was fixed at $10^{\circ}$. Blowing-momentum coefficients were chosen based on previous experimental results (ref. 7). To match the higher values of $C_{\mu}$, it was necessary to limit the free-stream Reynolds number to 100,000 . Testing at a higher Reynolds number could have been performed with a stronger blowing system, but a previous experiment demonstrated that the unsteady-load coefficients on a VR-7 do not vary appreciably within the Reynolds-number capability of the facility (ref. 8).

Steady-state loads were not measured in the experiment. Previous experiments have shown that loads measured at very low reduced frequencies (quasisteady loads) are approximately the same as true steady-state loads (ref. 9). All force and moment data were reduced to non-dimensional coefficient form during processing. Lift, drag, and pitching-moment coefficients are shown in figures 9 through 34 with angle of attack and $\omega t$ as the independent variables. The conditions for each test point are shown in table 2 .

While every effort was made to record data at nominal conditions, some variation in Reynolds number, $R e$, reduced frequency, $k$, and momentum coefficient, $C_{\mu}$, were observed. This variation is the result of using the mean dynamic pressure to compute $V_{\infty}$, which appears in each of the above coefficients. This quantity varies from cycle to cycle because of the unsteady test-section blockage introduced by the airfoil's motion. In addition, the actual values of $Q_{s}$ were also slightly different from the nominal values attempted.

Flow-visualization photographs were obtained for several points in each cycle. The photographs for all quasi-steady oscillations are sequenced according to angle-of-attack values instead of wt since the flow field is not appreciably different from a true steadystate condition. Photographs for oscillations at higher reduced frequencies are sequenced according to values of $\omega t$. The correspondence between the angle of attack and a particular $\omega t$ depends on the mean angle of attack and is governed by $\alpha=\alpha_{m}+10^{\circ} \sin \omega t$. Keeping this relationship in mind will reduce confusion when viewing the visualization photographs.

\section{Steady Blowing with $\boldsymbol{\alpha}_{m}=10^{\circ}$ and $k=0.005$ (nominal)}

Zero-blowing reference- The lowest reduced frequency tested at $\alpha_{m}=10^{\circ}$ was $k=0.004$. This corresponds to a quasi-steady oscillation rate of approximately $0.017 \mathrm{~Hz}$. Figure 9(a) shows the flowvisualization results with no blowing. The photographs show a progressive increase in the boundary-layer thickness between $\alpha=5^{\circ}$ and $12^{\circ}$. At $\alpha=12^{\circ}$, some initial trailing-edge separation is visible. The separation point steadily works its way forward and reaches the leading edge at an angle of attack near $16^{\circ}$.

Quantitative results for the quasi-steady oscillation with no blowing are shown in figure 9(b). The lift coefficient is approximately linear up to an angle of attack near $12^{\circ}$. The lift-curve slope then decreases to nearly zero as trailing-edge separation spreads over the surface. As the separation point nears the leading edge, a spike in the lift coefficient appears near $16^{\circ}$. This is probably the result of a small vortex shedding as the boundary layer fully separates. The spike is followed by a sharp decrease in the lift coefficient. Pitching moment is also approximately linear up to the appearance of flow separation $\left(\alpha=12^{\circ}\right)$. Stall brings an impulsive nose-down jump in the moment. Drag is roughly quadratic until the point of stall $\left(\alpha=16^{\circ}\right)$, where it increases suddenly. All the curves have mild hysteresis between $\alpha=12^{\circ}$ and $16^{\circ}$, the range where flow separation is present.

Low-blowing rate- When blowing at $C_{\mu}=0.16$ was applied to the quasi-steady oscillation, several changes were observed. Figure 10(a) shows the flowvisualization results for the cycle. The photographs reveal the development of a separation bubble near $x / c=0.125$. The bubble is first visible at $\alpha=15^{\circ}$. As the angle of attack continues to increase, the boundary layer over the rest of the airfoil becomes very thick. At $\alpha=17^{\circ}$, the boundary layer looks highly turbulent over most of the surface. A substantial region of trailing-edge separation is visible at $\alpha=18^{\circ}$.

Quantitative results for the cycle are shown in figure 10 (b). Blowing at $C_{\mu}=0.16$ removes almost all the hysteresis in the curves. The lift coefficient is roughly linear up to $\alpha=10^{\circ}$. The slope then decreases slightly as the separation bubble develops. Near $\alpha=17^{\circ}$, the slope of the lift curve changes to 
Table 2. Steady-blowing test conditions

\begin{tabular}{rrrrrrrr}
\hline \hline Figure & Frame & $\alpha_{m}$ & $k$ & $\begin{array}{c}Q_{s}, \\
\mathrm{gpm}\end{array}$ & $C_{\mu}$ & $\begin{array}{c}\text { Cycles } \\
\text { averaged }\end{array}$ & $V_{s} / V_{\infty}$ \\
\hline 9 & 10299 & $10^{\circ}$ & 0.004 & 0.0 & 0.0 & 5 & 0.0 \\
10 & 10306 & $10^{\circ}$ & 0.005 & 2.7 & 0.16 & 5 & 10.3 \\
11 & 10311 & $10^{\circ}$ & 0.006 & 4.9 & 0.54 & 5 & 19.0 \\
& & & & & & & \\
13 & 10302 & $10^{\circ}$ & 0.05 & 0.0 & 0.0 & 30 & 0.0 \\
14 & 10307 & $10^{\circ}$ & 0.05 & 2.7 & 0.16 & 30 & 10.3 \\
15 & 10312 & $10^{\circ}$ & 0.05 & 4.9 & 0.57 & 30 & 19.5 \\
& & & & & & & \\
17 & 10303 & $10^{\circ}$ & 0.15 & 0.0 & 0.0 & 30 & 0.0 \\
18 & 10308 & $10^{\circ}$ & 0.15 & 2.7 & 0.16 & 30 & 10.3 \\
19 & 10313 & $10^{\circ}$ & 0.15 & 4.9 & 0.56 & 30 & 19.3 \\
& & & & & & & \\
21 & 10215 & $15^{\circ}$ & 0.007 & 0.0 & 0.0 & 5 & 0.0 \\
22 & 10225 & $15^{\circ}$ & 0.007 & 2.7 & 0.19 & 5 & 11.3 \\
23 & 10222 & $15^{\circ}$ & 0.006 & 4.9 & 0.64 & 5 & 20.7 \\
& & & & & & & \\
25 & 10216 & $15^{\circ}$ & 0.05 & 0.0 & 0.0 & 30 & 0.0 \\
26 & 10226 & $15^{\circ}$ & 0.05 & 2.7 & 0.19 & 30 & 11.3 \\
27 & 10221 & $15^{\circ}$ & 0.05 & 4.9 & 0.66 & 30 & 21.0 \\
29 & 10217 & $15^{\circ}$ & 0.15 & 0.0 & 0.0 & 30 & 0.0 \\
30 & 10227 & $15^{\circ}$ & 0.15 & 2.7 & 0.19 & 30 & 11.3 \\
31 & 10220 & $15^{\circ}$ & 0.15 & 4.9 & 0.65 & 30 & 20.8 \\
\hline \hline
\end{tabular}

a slightly negative value for the rest of the upstroke. The slope change is almost instantaneous, indicating the sudden appearance of flow separation. The pitching moment is approximately linear to $\alpha=12.5^{\circ}$. The slope levels off as the separation bubble develops and then drops off sharply with the onset of separation. The drag also undergoes a large change in slope when the boundary layer separates. A significant amount of thrust is produced by blowing, causing a downward shift in the drag curve. As a result, the drag coefficient is negative for about half of the cycle.

High-blowing rate- Blowing at $C_{\mu}=0.54$ eliminates massive separation on the airfoil. Figure 11(a) shows the flow-visualization results for the cycle. A separation bubble forms at this blowing rate also. The bubble is initially visible at $\alpha=15^{\circ}$. As the airfoil's incidence continues to increase, the size of the separation bubble increases, and the boundary layer becomes very thick. Large, turbulent structures are visible in the boundary layer at the maximum angle of attack
( $\alpha=20^{\circ}$ ). No trailing-edge separation is visible in the photographs.

Quantitative results for the cycle are shown in figure 11(b). None of the curves show any evidence of stall at this blowing rate. All coefficients reach a maximum at the angle-of-attack limit $\left(\alpha=20^{\circ}\right)$. Hysteresis is noticeable in the drag curve only. The lift and moment curves are approximately linear up to an angle of attack near $18^{\circ}$. Minor slope changes occur at this point, and the new slopes are maintained for the rest of the upstroke. The drag coefficient behaves smoothly throughout the cycle. Drag coefficients are negative for $\alpha<17.5^{\circ}$ due to jet thrust.

Comparison of results- Figure 12 shows a comparison of the loads presented in figures 9-11. It shows that higher blowing rates produce progressively higher lift-curve slopes. The slope increases by $23 \%$ for $C_{\mu}=0.16$ and by $28 \%$ for $C_{\mu}=0.54$. Blowing also increases the maximum-lift coefficient and delays boundary-layer separation. Separation is delayed by about $5^{\circ}$, and the maximum-lift coefficient is increased 
by $38 \%$ for $C_{\mu}=0.16$. Blowing at $C_{\mu}=0.54$ prevents separation and increases the maximum-lift coefficient by $75 \%$. Both blowing rates eliminate the small lift spike that appears near $\alpha=16^{\circ}$ without blowing. Lift hysteresis also disappears, for all practical purposes, for both blowing rates.

The most noticeable effect of blowing on drag is a push toward negative coefficients resulting from jet thrust. Blowing at $C_{\mu}=0.16$ produces a negative drag coefficient for half of the cycle. Blowing at $C_{\mu}=$ 0.54 lowers the drag coefficient slightly below -0.2 at $\alpha=0^{\circ}$, and it causes negative coefficients to occur for all but $2.5^{\circ}$ of incidence during the cycle. The sharp drag increase at the onset of stall does not appear for either of the blowing cases. However, a small amount of hysteresis is present for both blowing rates.

Blowing produces some interesting changes in the quarter-chord moment. The positive shift that occurs at $\alpha=0^{\circ}$ is probably caused by an increase in the velocity of the flow over the leading edge. A high-speed jet tends to drag the surrounding flow with it through viscous interaction. Part of the surrounding flow in this situation is made up of fluid that previously formed the leading-edge boundary layer. Bearing in mind that liquid water is considered incompressible, mass conservation requires that the average flow speed in the leading-edge boundary layer must increase to compensate for a higher average flow speed next to the jet. The end result is more leading-edge suction. Since the pressure is lowered in front of the reference axis for the pitching moment, the moment coefficient becomes more positive. Another interesting change that occurs with increasing blowing rate is the progressive increase in the magnitude of the slope of the pitching-moment curves for $\alpha<13^{\circ}$ and a decrease in the magnitude of the pitching moments at high angles of attack. Moment hysteresis is reduced to negligible levels at both blowing rates.

\section{Steady Blowing with $\alpha_{m}=10^{\circ}$ and $k=0.05$}

Zero-blowing reference- Figure 13(a) shows the flow-visualization results for the unsteady oscillation with $k=0.05$ and no blowing. The dynamic stall that is observed in this case is considered to be limited; that is, massive separation only occurs over a small portion of the cycle. These photographs show the rapid appearance of reversed flow as the airfoil rotates from $\omega t=20^{\circ}$ to $30^{\circ}$. At $\omega t=40^{\circ}$, a dynamic-stall vortex is visible over the quarter-chord point, and the aft portion of the boundary layer is coalescing. The photograph for $\omega t=45^{\circ}$ shows the dynamic-stall vortex migrating over the upper surface near $x / c=0.5$, along with a large structure that is visible over the trailing edge. By the time the airfoil reaches $\omega t=50^{\circ}$, the dynamic-stall vortex has been shed from the trailing edge, and the boundary layer has separated from the leading edge. The boundary layer does not begin to re-form until $\omega t=135^{\circ}$. At $\omega t=225^{\circ}$, the boundary layer has been reestablished.

Figure 13(b) contains quantitative results for the cycle. The figure shows that the lift and moment curves are approximately linear up to an angle of attack near $13^{\circ}\left(\omega t=17^{\circ}\right)$. Between $\alpha=13^{\circ}$ and $17^{\circ}\left(\omega t=17^{\circ}\right.$ and $44^{\circ}$ ), the lift-curve slope decreases to nearly zero as reversed flow spreads over the surface. A sudden distortion in the lift and moment curves, caused by the formation and movement of the dynamic-stall vortex, begins near $\alpha=17^{\circ}\left(\omega t=44^{\circ}\right)$. The figure shows that moment stall occurs before the lift stall. Large hysteresis amplitudes are present in all the loads during the downstroke and persist until the angle of attack is below $\alpha=10^{\circ}\left(\omega t=180^{\circ}\right)$. An interesting feature of the moment curve is the small region with a clockwise orientation that is present between $\alpha=15^{\circ}\left(\omega t=30^{\circ}\right)$ and $\alpha=18^{\circ}\left(\omega t=53^{\circ}\right)$. A clockwise orientation represents a region of negative pitch damping. This is an unstable region where energy is being extracted from the flow (ref. 1). A pitch-damping term, $\zeta$, is mathematically defined by

$$
\zeta=-\oint \frac{C_{m} d \alpha}{4 \alpha^{2}}
$$

Low-blowing rate- Figure 14(a) shows the flowvisualization results for the unsteady oscillation with $k=0.05$ and $C_{\mu}=0.16$. A separation bubble begins to form over $x / c=0.125$ at $\omega t=30^{\circ}$. At $\omega t=$ $45^{\circ}$ and $50^{\circ}$, large, turbulent structures are seen in the boundary layer, and the jet is beginning to separate from the surface. The size of the separation bubble has also increased significantly. The frame for $\omega t=60^{\circ}$ shows that the jet has separated from the surface.

When the jet fully separates from the surface, it prevents closure of the leading-edge bubble. The frame for $\omega t=70^{\circ}$ shows that a "vortex-like" structure is present near the point where the jet and the leading-edge boundary layer meet $(x / c=0.5)$. Researchers believe that this structure was released when the leading-edge bubble was compromised. The frame 
for $\omega t=80^{\circ}$ shows the flow separated from the leading edge. The jet remains separated as well.

The first frame showing the flow during the downstroke is $\omega t=135^{\circ}$. In this frame the adverse pressure gradient appears to subside slightly because the jet flow is now able to push itself further toward the trailing edge before lifting off the surface. The size of the separated zone also appears to be smaller. By the time the airfoil reaches $\omega t=180^{\circ}$, a boundary layer has been completely reestablished over the entire upper surface.

Quantitative results for the cycle are shown in figure 14(b). The lift and moment curves are approximately linear between $\alpha=0^{\circ}$ and $15^{\circ}\left(\omega t=-90^{\circ}\right.$ and $30^{\circ}$ ). Jet thrust shifts the drag curve downward about as much as it did in figure 10(b) for the quasisteady case. Between $\alpha=15^{\circ}\left(\omega t=30^{\circ}\right)$ and $\alpha=18^{\circ}\left(\omega t=53^{\circ}\right)$, the slopes of the lift and moment curves begin to change, corresponding to the development of the leading-edge bubble and the initial jet separation from the surface. As the jet fully separates, all the loads change rapidly. The maximum negativepitching moment corresponds to the presence of the vortex-like structure seen in the frame for $\omega t=70^{\circ}$. When compared to the zero-blowing case, load hysteresis is reduced, and the moment curve maintains a counter-clockwise orientation throughout the cycle.

High-blowing rate- Figure 15(a) shows the flowvisualization results for the unsteady oscillation with $k=0.05$ and $C_{\mu}=0.57$. At this blowing rate, trailing-edge separation appears to be restrained during the cycle. Similar to the previous cycles with blowing, a separation bubble starts to form at $\omega t=30^{\circ}$. The bubble reaches its maximum size near $\omega t=70^{\circ}$, after which the bubble fails to close. At this point, the boundary layer is also very thick and contains large turbulent structures. No vortex-like structures are noticeable on the surface after the bubble is compromised. The jet appears to be slightly separated from the surface at $\omega t=90^{\circ}$. As the airfoil's angle of attack decreases, the separation bubble re-forms and is no longer visible at $\omega t=180^{\circ}$.

Quantitative results for the cycle are shown in figure 15(b). The load behavior shows some improvement over the results for the low-blowing rate shown in figure 14(b). The lift and moment curves are approximately linear throughout most of the cycle, and the drag curve shows a more gentle variation with angle of attack. There is a substantial downward shift in the drag curve due to the jet thrust. The magnitude of the drag shift is similar to that shown in figure 11(b) for the quasi-steady case. Lift hysteresis is reduced at this higher value of momentum coefficient. The moment curve maintains a counter-clockwise orientation throughout the cycle.

Comparison of results- Figure 16 shows a comparison of the loads obtained for $\alpha_{m}=10^{\circ}$ and $k=0.05$. The blowing results for this case appear to be qualitatively similar to those obtained at $k=0.005$ (fig. 12). The magnitudes of the hysteresis amplitudes are generally much larger for this case. However, it is clear that an increase in the momentum coefficient causes the lift hysteresis to be progressively reduced. Although not as much as in the quasi-steady case, the lift-curve slope during the upstroke increases by $17 \%$ for $C_{\mu}=0.16$ and by $26 \%$ for $C_{\mu}=0.57$. Stall is also progressively delayed as stronger blowing is applied. Blowing at $C_{\mu}=0.16$ results in a $35 \%$ increase in the maximum-lift coefficient, whereas blowing at $C_{\mu}=0.57$ results in a $59 \%$ increase. Blowing at both rates removes the distortion (the flat zone followed by a surge) in the lift curve prior to stall.

Changes in the pitching moment are similar to those at $k=0.005$. As the blowing rate is increased, the moment coefficients increase (become more positive) at low angles of attack, the magnitudes of the moment-curve slopes progressively increase, and the peak moment coefficients are decreased. Blowing at $C_{\mu}=0.57$ reduces the peak moment by $40 \%$.

\section{Steady Blowing with $\boldsymbol{\alpha}_{m}=10^{\circ}$ and $k=0.15$}

Zero-blowing reference- Figure 17(a) shows the flow-visualization results for the unsteady oscillation with $k=0.15$ and no blowing. The airfoil experienced deep stall at these conditions. Reversed flow appears slightly before $\omega t=40^{\circ}$. Formation of the dynamic-stall vortex begins near $\omega t=45^{\circ}$. At $\omega t=70^{\circ}$. the dynamic-stall vortex is visible as it moves over the surface near $x / c=0.5$. When the airfoil reaches $\omega t=90^{\circ}$, the boundary layer has completely separated. Additionally, the frame for $\omega t=90^{\circ}$ faintly shows the dynamic-stall vortex over the trailing edge along with a secondary vortex near the quarter chord. The boundary layer has been reestablished by $\omega t=225^{\circ}$.

Quantitative results for the cycle are shown in figure 17(b). The lift and moment coefficients are approximately linear up to $\alpha=16^{\circ}\left(\omega t=37^{\circ}\right)$. At this angle the two curves distort because of the spread of reversed 
flow and the presence of the dynamic-stall vortex. Moment stall precedes lift stall by a few degrees. Large hysteresis amplitudes are present in the loads during the entire downstroke, and a region of clockwise orientation is present in the moment curve between $\alpha=17^{\circ}$ $\left(\omega t=44^{\circ}\right)$ and $\alpha=19^{\circ}\left(\omega t=64^{\circ}\right)$.

Low-blowing rate- Figure 18(a) shows the flowvisualization results for the unsteady oscillation with $k=0.15$ and $C_{\mu}=0.16$. At this reduced frequency, a separation bubble begins to form near $x / c=0.125$ at $\omega t=50^{\circ}$. As figure 14(a) showed for $k=0.05$, the jet separates from the surface and hinders the closure of the leading-edge bubble. At $\omega t=80^{\circ}$, the jet is separated from the surface and a vortex-like structure is present near $x / c=0.5$. This structure appears to have been released when the bubble was compromised. The boundary layer over the upper surface begins to reestablish itself near $\omega t=135^{\circ}$.

Quantitative results for the cycle are shown in figure 18(b). The lift coefficient is approximately linear up to an angle of attack near $\alpha=18^{\circ}\left(\omega t=53^{\circ}\right)$, then the lift slope decreases smoothly through the peak angle of attack. The initial decrease does not appear to be related to separation since none is visible in the photographs for $\omega t=50^{\circ}$ or $60^{\circ}$. The likely cause for this decrease is the change in relative flow velocity caused by the deceleration of the airfoil. Separation is, however, the leading cause of the hysteresis amplitudes that are present throughout the downstroke. Overall, this cycle has less lift hysteresis than the cycle without blowing. A distortion near $\alpha=13^{\circ}\left(\omega t=17^{\circ}\right)$ in the downstroke indicates that the jet is forcing the boundary layer to re-form.

The drag and pitching moment curves do not show as large a reduction of hysteresis. The moment hysteresis does not show any negative damping with blowing. Jet thrust appears as a downward shift in the drag curve. The effect is similar in magnitude to the effects observed at $k=0.005$ and 0.05 . The moment stall that occurs for this cycle is not as severe as that which occurs for the cycle without blowing. As shown by $k=0.05$ with light blowing, the maximum negativepitching moment corresponds to the presence of the vortex-like structure seen in the frame for $\omega t=80^{\circ}$.

High-blowing rate-Figure 19(a) shows the flowvisualization results for the unsteady oscillation with $k=0.15$ and $C_{\mu}=0.56$. This blowing rate prevented any significant trailing-edge separation from occurring. A separation bubble begins to form at approximately $\omega t=45^{\circ}$. It reaches its maximum size at $\omega t=70^{\circ}$ where it covers roughly $25 \%$ of the upper surface. Beyond this angle, it appears that the bubble has opened up at the rear; however, the leading-edge separation remains somewhat restrained. No vortex-like structures are observed at this blowing rate. The boundary layer has returned to its undisturbed state by $\omega t=180^{\circ}$.

Quantitative results for the cycle are shown in figure 19(b). They are quite similar to the results obtained for the low blowing rate. One exception is a larger hysteresis reduction in the lift at the higher blowing rate. Another difference is the elimination of moment stall. The drag offset is due to jet thrust and is similar to that observed at the lower reduced frequencies. The moment curve continues to maintain a counter-clockwise orientation (positive pitch damping) throughout the cycle.

Comparison of results- Figure 20 is a comparison of the loads obtained for $\alpha_{m}=10^{\circ}$ and $k=0.15$. The changes produced by blowing are similar to those observed at $k=0.005$ and $k=0.05$ (figs. 12 and 16). Increasing the blowing rate progressively reduces the lift hysteresis. However, the reductions are not as large as they were at the previous reduced frequencies. The lift-curve slope during the upstroke increases by $12 \%$ for $C_{\mu}=0.16$ and $20 \%$ for $C_{\mu}=0.56$. Increases in the momentum coefficient progressively delay the onset of stall. The maximum-lift coefficient increases $18 \%$ for $C_{\mu}=0.16$ and $30 \%$ for $C_{\mu}=0.56$. The distortion in the lift curve prior to stall disappears at both blowing rates.

The changes to the drag and moment are also similar to those obtained at the lower reduced frequencies. A thrust effect is visible in the drag curve. The magnitude of the effect appears to be about the same as that seen previously. The moment coefficient increases at $\alpha=0^{\circ}$, and the magnitude of the slope becomes more negative with stronger blowing. The maximum negative moment decreases by $43 \%$ for $C_{\mu}=0.56$. Similar to the previous reduced-frequency case, a vortex-like structure is observed at the lowest blowing rate. The presence of this structure corresponds to the maximum nose-down moment. The structure is not seen at the highest blowing rate. Blowing at both rates forces the moment curve to maintain a counter-clockwise orientation throughout the cycle. Neither moment nor drag hysteresis is significantly reduced with blowing. 
Steady Blowing with $\alpha_{m}=15^{\circ}$ and $k=0.007$ (nominal)

The flow-visualization results for the quasi-steady oscillations about $\alpha_{m}=15^{\circ}$ are presented in figures 21(a), 22(a), and 23(a). In each case the sequence of boundary-layer events between $\alpha=10^{\circ}$ and $20^{\circ}$ is identical to the events observed for $\alpha_{m}=10^{\circ}$. Beyond $20^{\circ}$, the boundary layer completely separates from the leading edge during each cycle. Quantitative results are shown in figures $21(\mathrm{~b}), 22(\mathrm{~b})$, and 23(b). The results are compared in figure 24 . Like the flowvisualization results, the general behavior of the loads is virtually identical to the behavior at $\alpha_{m}=10^{\circ}$. The only differences are lower post-stall values. No significant hysteresis is present during the oscillations with blowing.

\section{Steady Blowing with $\boldsymbol{\alpha}_{m}=15^{\circ}$ and $k=0.05$}

Zero-blowing reference- Figure 25(a) shows the flow-visualization results for the unsteady oscillation with $k=0.05$ and no blowing. The airfoil experienced deep dynamic stall at these conditions. The frame for $\omega t=10^{\circ}$ shows a thick boundary layer with reversed flow over the trailing edge. The next frame $\left(\omega t=20^{\circ}\right)$ shows that a dynamic-stall vortex has already formed and has moved to approximately $x / c=0.75$. The photograph for $\omega t=30^{\circ}$ shows the boundary layer completely separated from the leading edge and the dynamic-stall vortex convecting downstream in the wake. A large secondary vortex is visible over the quarter-chord point. A boundary layer is beginning to be reestablished over the forward $30 \%$ of the airfoil at $\omega t=180^{\circ}$.

Quantitative results for the cycle are shown in figure 25(b). The lift curve is approximately linear up to an angle of attack near $\alpha=13^{\circ}\left(\omega t=-17^{\circ}\right)$. At this point the familiar distortion appears in the lift curve. The distortion and the stall are spread over a greater range at this mean angle because the airfoil has a higher rotational speed, $d \alpha / d t$, through the stall angle. Like the cycles at the lower mean angle, moment stall precedes lift stall by a few degrees. Additionally, since the airfoil spends nearly one third of its cycle beyond the stall angle, much more hysteresis appears in the loads during the downstroke. The moment curve maintains a counter-clockwise orientation throughout the cycle.

Low-blowing rate-Figure 26(a) shows the flowvisualization results for the unsteady oscillation with $k=0.05$ and $C_{\mu}=0.19$. A separation bubble appears near $x / c=0.125$ at $\omega t=10^{\circ}$. At $\omega t=30^{\circ}$, the jet is unable to overcome the adverse pressure gradient and separates from the surface. A vortex-like structure is present in the frame at the point where the separated leading-edge flow and the jet flow meet (near $x / c=0.5$ ). A wake vortex is also present just off the trailing edge. As the airfoil continues its upward rotation, the vortex-like structure is convected from the airfoil, and the jet partially reattaches itself to approximately $x / c=0.75$. The boundary layer is reestablished when the airfoil reaches $\omega t=180^{\circ}$.

Quantitative results for the cycle are shown in figure 26(b). The lift and moment curves are approximately linear between $\alpha=5^{\circ}$ and $19^{\circ}\left(\omega t=-90^{\circ}\right.$ and $24^{\circ}$ ). The drag behavior is roughly quadratic in this range. Jet thrust appears in the drag curve for the lower angles of attack and is similar in magnitude to the thrust observed when $\alpha_{m}=10^{\circ}$. As the airfoil rotates through $\alpha=19^{\circ}\left(\omega t=24^{\circ}\right)$, the lift and moment coefficients begin to decrease rapidly. This corresponds to the separation of the jet from the surface and the presence of the vortex-like structure at $\alpha=30^{\circ}$. Blowing removes some of the hysteresis in all the loads by forcing the boundary layer to re-form near $\alpha=15^{\circ}\left(\omega t=180^{\circ}\right)$.

High-blowing rate- Figure 27(a) shows the flowvisualization results for the unsteady oscillation with $k=0.05$ and $C_{\mu}=0.66$. The photographs show a separation bubble developing between $\omega t=10^{\circ}$ and $30^{\circ}$. The boundary layer over the trailing edge also thickens during this period. The frame for $\omega t=40^{\circ}$ shows a vortex-like structure being shed from the separation bubble. Between $\omega t=50^{\circ}$ and $135^{\circ}$, the boundary layer is completely separated from the leading edge. The jet flow appears to be separated only in the frame for $\omega t=50^{\circ}$. The sequence of photographs for $\omega t=30^{\circ}$ through $\omega t=60^{\circ}$ indicates that jet separation may be influenced by the presence of the vortex-like structure. At $\omega t=180^{\circ}$, it appears that the boundary layer has been reestablished on the airfoil.

Quantitative results for the cycle are shown in figure 27 (b). The load behavior is similar to that for the low blowing case shown in figure 26(b). The lift and moment curves are approximately linear up to $\alpha=20^{\circ}$ $\left(\omega t=30^{\circ}\right)$, after which the stall occurs suddenly. Moment stall appears to be much more severe at this blowing rate, and it also appears to be coincident with the 
migration of the vortex-like structure. Further examination of the moment curve shows less hysteresis and a smaller amount of negative damping compared with the results at the lower blowing rate.

Comparison of results- Figure 28 shows a comparison of the loads obtained at $\alpha_{m}=15^{\circ}$ and $k=0.05$. The effects of blowing are similar to those observed in the quasi-steady cases summarized in figure 24 and in the low-amplitude cases summarized in figure 16. The primary differences involve the amount of hysteresis present and the maximum nose-down moment.

The increase in hysteresis that occurs at this pitching rate $(k=0.05)$ can be attributed to the finite amount of time required by the flow to adjust to the motion of the airfoil. The increase in the magnitude of the pitching moment seen at this mean angle of attack $\left(\alpha_{m}=15^{\circ}\right)$ is probably due to the formation of a much stronger, vortex-like structure. In other words, as the blowing rate is increased, the separation bubble is trapped at the leading edge for a longer period of time. This would allow the vorticity within the bubble to gain more strength. If the blowing rate is insufficient to maintain the bubble and a vortex-like structure is released, the structure will migrate over the surface and produce a change in the pressure distribution. A stronger vortex will change the pressure distribution even more and cause a larger nose-down moment.

\section{Steady Blowing with $\alpha_{m}=15^{\circ}$ and $k=0.15$}

Zero-blowing reference- Figure 29(a) shows the flow-visualization results for the unsteady oscillation with $k=0.15$ and no blowing. A dynamic-stall vortex is beginning to form in the frame for $\omega t=20^{\circ}$. Vortex growth and migration is shown in the frames for $\omega t=30^{\circ}$ to $60^{\circ}$. At $\left.\omega t=60\right)^{\circ}$, the stall vortex has moved about half a chord length off the surface, and the boundary layer has separated from the leading edge. A secondary vortex is visible in the next frame. The leading-edge separation persists through $\omega t=135^{\circ}$. At $\omega t=180^{\circ}$, the separation point has moved rearward to the approximate quarter-chord location. The boundary layer is reestablished by the time the airfoil reaches $\omega t=270^{\circ}$.

Quantitative results for the cycle are shown in figure 29(b). The unsteady loads indicate that the airfoil experiences a "textbook case" of deep stall at these conditions. The lift and moment curves deviate from the approximate linear behavior that has been observed in previous cases at the lower angles of attack. All regions of the two curves, except for the post-stall region, show some curvature. The familiar distortion in the lift and moment curves begins near $\alpha=19^{\circ}\left(\omega t=24^{\circ}\right)$ as a dynamic-stall vortex begins to grow and move over the surface of the airfoil. Moment stall occurs just before $\alpha=20^{\circ}\left(\omega t=30^{\circ}\right)$, and lift stall begins near $\alpha=23^{\circ}\left(\omega t=49^{\circ}\right)$. Hysteresis is present throughout the downstroke in all the loads. The moment curve maintains a counter-clockwise orientation throughout the cycle (positive-pitch damping).

Low-blowing rate- Figure 30(a) shows the flowvisualization results for the unsteady oscillation with $k=0.15$ and $C_{\mu}=0.19$. The photographs show the development of a separation bubble between $\omega t=20^{\circ}$ and $30^{\circ}$. At $\omega t=40^{\circ}$, the downstream boundary of the bubble is no longer closed. The frames for $\omega t=50^{\circ}$ through $135^{\circ}$ show events similar to those observed in the figures for a lower reduced frequency. The boundary layer is attached over much of the airfoil by $\omega t=180^{\circ}$, but it is still very thick. A final eddy structure from the separated region is visible above the trailing edge as the boundary layer re-forms on the forward portion of the airfoil. The boundary layer has thinned considerably, and no evidence of separation remains when the airfoil reaches $\omega t=225^{\circ}$.

Quantitative results for the cycle are shown in figure $30(\mathrm{~b})$. The lift and moment curves are slightly nonlinear between $\alpha=5^{\circ}$ and $20^{\circ}$ ( $\omega t=-90^{\circ}$ and $30^{\circ}$ ). The distortion in the lift curve that was observed prior to stall without blowing has been removed. Blowing causes the peak-load amplitudes to be delayed by about $2.5^{\circ}$ of airfoil incidence. Moment stall corresponds to the movement of the vortex-like structure over the surface. Lift hysteresis decreases near $\alpha=15^{\circ}\left(\omega t=180^{\circ}\right)$ because of the jet's influence on the boundary layer. Thrust appears in the drag curve and is similar in magnitude to the previous cases with $C_{\mu}=0.19$.

High-blowing rate- Figure 31(a) shows the flowvisualization results for the unsteady oscillation with $k=0.15$ and $C_{\mu}=0.65$. The photographs show the development of a separation bubble between $\omega t=10^{\circ}$ and $50^{\circ}$. The jet is able to maintain flow attachment over the trailing edge throughout this range. At $\omega t=$ $60^{\circ}$, the bubble is no longer closed and the edge of this zone is very irregular. The next frame $\left(\omega t=70^{\circ}\right)$ shows the vortex-like structure from the bubble being convected over the airfoil. The structure is still visible above the airfoil's surface at $\omega t=80^{\circ}$, and the flow 
is separated from the leading edge. At $\omega t=90^{\circ}$ the jet is separated from the surface.

Quantitative results for the cycle are shown in figure 31(b). The results show that a higher blowing rate causes an increase in the peak value of lift and a reduction in the hysteresis amplitude. The drag is significantly reduced throughout the cycle. The pitchingmoment hysteresis is not measurably reduced and a zone of negative damping is present.

Comparison of results- Figure 32 is a comparison of the loads obtained for $\alpha_{m}=15^{\circ}$ and $k=0.15$. The trends are similar to those observed at the lower mean angle and at the same reduced frequency (fig. 20). Increasing the blowing rate progressively reduces the lift hysteresis, increases the lift-curve slope during the upstroke, delays stall, and increases the maximum-lift coefficient. Blowing also removes the distortion in the lift curve prior to stall. The primary difference between the high-and low-amplitude results is that, as the blowing rate is increased in the high-amplitude case, the peak-pitching moment is not reduced and a state of positive pitch damping is not always assured. .

\section{Summary of Steady-Blowing Results}

Steady upper-surface blowing has several notable effects on the dynamic-stall behavior of the VR-7 airfoil. Blowing significantly enhances the lift by trapping a separation bubble at the leading edge during a portion of the upward rotation of the airfoil. Blowing removes the fluctuations in the loads caused by the appearance of reversed flow and the initial formation and movement of the dynamic-stall vortex. Whenever the dynamic-stall vortex can be trapped at the leading edge, moment stall and lift stall are eliminated. Even when upper-surface blowing is not strong enough to maintain the separation bubble for the entire cycle, a benefit is still realized through a substantial reduction of the load hysteresis. Blowing thrust was also observed. The best results were obtained at the lowest reduced frequencies, the lowest mean angle of attack, and the highest value of $C_{\mu}$.

In each case, the increases in lift were greater than the equivalent values of thrust associated with $C_{\mu}$ (non-dimensional jet momentum). Since $C_{\mu}$ can be interpreted as the thrust coefficient per unit span produced by blowing, the results show that tangential upper-surface blowing produces more additional lift than would have been generated had the jet been used as a vertical thruster. Drag reduction, on the other hand, was always less than $C_{\mu}$. Since the fluid from the jet had to travel over $75 \%$ of the upper surface before reaching the trailing edge of the airfoil, the momentum of the jet was reduced by friction so that the theoretical maximum-thrust coefficient, $C_{\mu}$, was not obtained.

Other interesting effects were also noted. For pitch oscillations about a mean angle of $10^{\circ}$, increasing the blowing strength progressively reduced the maximum negative-moment coefficient. This was not the case when the mean angle was $15^{\circ}$. Instead, stronger blowing resulted in a progressively larger negativemoment coefficient. This behavior is believed to be related to the strength of the vortex-like structures that were produced when the jet was no longer able to maintain the leading-edge bubble. These structures were observed at both mean angles, but they were much more defined at $\alpha_{m}=15^{\circ}$.

At $\alpha_{m}=10^{\circ}$, blowing reduced the strength of the vortex-like structures to the point where they did not cause a severe pitching moment during their movement over the surface. In fact, it appears that the structures were dissipated before they reached the trailing edge. At $\alpha_{m}=15^{\circ}$, blowing was unable to weaken the structures to any significant degree. Rather, the separation bubble was maintained at the leading edge where it gained strength. When the separation bubble finally became unstable and released the vortex-like structure, the pressure distribution on the airfoil was altered and a severe nose-down moment resulted. Higher blowing rates trapped the leading-edge bubbles longer, producing a stronger vortex structure and a larger negativepitching moment.

Blowing also increased the moment coefficient at $\alpha=0^{\circ}$ and caused a decrease in the magnitude of the moment-curve slope. The authors believe that this behavior is the result of the jet dragging the surrounding fluid by viscous interaction, and subsequently, increasing the flow speed over the leading edge.

The beneficial changes to the unsteady loads that are derived from upper-surface blowing are countered by increases in either the reduced frequency or the mean angle of oscillation. Figure 33 shows how the hysteresis loops are expanded as the reduced frequency is increased. The peak negative moment also increases slightly. Figure 34 shows how the lift hysteresis is greatly increased and how the maximum negative moment nearly doubles as the mean angle of oscillation is increased. 
A first-order analysis of the power required to achieve a given lift coefficient reveals that blowing (in the present setup) is an inefficient means of enhancing the performance of this airfoil, especially when compared with the results for a slatted airfoil reported in reference 8 . For example, comparing these two airfoils at $k=0.005$ and $C_{l}=1.3$ indicates that the airfoil with blowing requires approximately 19 times more power than the slatted airfoil. This comparison was made on the basis of $q_{\infty} c C_{d} V_{\infty}$, the power required to overcome airfoil drag, for a given $q_{\infty} c$. Although the drag was approximately zero for the airfoil with blowing, the power required to produce the blowing jet was equal to $q_{\infty} c C_{\mu} V_{s}$.

\section{PULSED-BLOWING RESULTS}

A preliminary investigation of the effects of pulsed blowing was also conducted. Unsteardy loads were measured on the oscillating airfoil for a range of pulsed-blowing conditions. The investigation was conducted at a Reynolds number of 100,000 for an oscillatory motion defined by $\alpha=10^{\circ}+10^{\circ} \sin \omega t$. A blowing-momentum coefficient, $C_{\mu}$, of 0.03 was chosen for this portion of the investigation, based on the results reported in reference 10 . The range of reduced frequencies of pulsing (nominally $F^{+}=0,1,2,3$ ) was also based on results reported in reference 10 . As with the steady-blowing study, quasi-steady loads were used in lieu of true steady-state loads. The conditions for each data set are shown in table 3 . Force and moment data were reduced to non-dimensional coefficient form during processing. Lift, drag, and pitching-moment coefficients are shown in figures 35 through 52 with $\alpha$ and $\omega t$ as the independent variables.

Two points must be mentioned about the conditions shown in table 3. First, even though the blowing momentum coefficient, $C_{\mu}$, and the dimensionless pulsing frequencies, $F^{+}$, were chosen because of the results of reference 10 , there was no assurance that these conditions would produce successful results for controlling dynamic stall. One reason is that the unsteady flow field during dynamic stall is significantly different from that during a quasi-steady stall. Another reason is that the blowing location for effective trapping of the stall vortex is not necessarily the best location for efficiently introducing flow perturbations aimed at preventing trailing-edge separation. Reference 10 merely provided a convenient starting point for this investigation.

\section{Pulsed Blowing with $k=0.006$ (nominal)}

Figures 35-38 show the results of pulsed blowing for a constant momentum coefficient $\left(C_{\mu}=0.024\right.$ nominal) and a fixed quasi-steady oscillation rate $(k=$ 0.006 nominal). Each figure represents a different reduced pulsing frequency, $F^{+}$. All the curves are very similar to each other, suggesting that there is little benefit from pulsed blowing at these conditions. Pulsed blowing also causes a fair amount of unsteadiness in the loads and is responsible for the jagged appearance of the curves.

The pulsed-blowing results are compared in figure 39. The figure indicates that a dimensionless pulsing frequency of $F^{+}=0.9$ offers the greatest improvement when compared to the steady-blowing case. The lift slope is approximately $1 \%$ greater than the steady-blowing case, and the maximum-lift coefficient is $7 \%$ larger.

Figure 40 presents a comparison of the loads with no blowing, steady blowing, and pulsed blowing at $F^{+}=0.9$, with each blowing type having approximately the same $C_{\mu}$. The decrease in the lift slope caused by the spread of trailing-edge separation is still present to some degree with steady blowing; however, the decrease is not observed for pulsed blowing at $F^{+}=0.9$. Both blowing types remove the spike in the lift curve prior to stall. Pulsed blowing provides a $20 \%$ increase in the maximum-lift coefficient, whereas steady blowing produces only a $12 \%$ increase. The lift increase for $F^{+}=0.9$ is similar in magnitude to the increases reported in references 12 and 13. Some hysteresis reduction occurs for each type of blowing, but pulsed blowing at $F^{+}=0.9$ is the most effective.

\section{Pulsed Blowing with $k=0.05$}

Figures 41-44 show the results of pulsed blowing at different dimensionless pulsing frequencies, a constant momentum coefficient ( $C_{\mu}=0.024$ nominal), and a fixed reduced frequency $(k=0.05)$. All the curves are very similar to each other, again indicating that there is little advantage to be gained from pulsed blowing at these conditions. The individual curves are compared in figure 45 . The figure shows that pulsed 
Table 3. Pulsed-blowing test conditions

\begin{tabular}{cccccccc}
\hline \hline Figure & Frame & $\alpha_{m}$ & $k$ & $C_{\mu}$ & $F^{+}$ & $\begin{array}{c}\text { Cycles } \\
\text { averaged }\end{array}$ & $V_{s} / V_{\infty}$ \\
\hline 35 & 10352 & $10^{\circ}$ & 0.006 & 0.023 & 0.0 & 5 & 3.9 \\
36 & 10356 & $10^{\circ}$ & 0.005 & 0.024 & 0.9 & 5 & 4.0 \\
37 & 10355 & $10^{\circ}$ & 0.006 & 0.023 & 1.9 & 5 & 3.9 \\
38 & 10357 & $10^{\circ}$ & 0.006 & 0.024 & 2.7 & 5 & 4.0 \\
& & & & & & & \\
41 & 10386 & $10^{\circ}$ & 0.05 & 0.025 & 0.0 & 30 & 4.1 \\
42 & 10366 & $10^{\circ}$ & 0.05 & 0.024 & 0.9 & 30 & 4.0 \\
43 & 10364 & $10^{\circ}$ & 0.05 & 0.023 & 1.9 & 30 & 3.9 \\
44 & 10362 & $10^{\circ}$ & 0.05 & 0.024 & 2.7 & 30 & 4.0 \\
47 & 10389 & $10^{\circ}$ & 0.15 & 0.025 & 0.0 & 30 & 4.1 \\
48 & 10373 & $10^{\circ}$ & 0.15 & 0.024 & 0.9 & 30 & 4.0 \\
49 & 10375 & $10^{\circ}$ & 0.15 & 0.024 & 1.9 & 30 & 4.0 \\
50 & 10376 & $10^{\circ}$ & 0.15 & 0.024 & 2.7 & 30 & 4.0 \\
\hline \hline
\end{tabular}

blowing at $F^{+}=0.9$ causes a slightly better hysteresis reduction and a marginally higher increase in the maximum-lift coefficient.

Figure 42(a) is the flow visualization for a representative cycle with pulsed blowing. The case for $F^{+}=0.9$ was selected since it provided the best results. The photographs for $\omega t=10^{\circ}$ through $30^{\circ}$ show that a discrete disturbance is present either on the airfoil surface or in the wake. There appears to be no more than one disturbance on the surface at any given time. Reference 12 indicated that one or two disturbances would yield the best results. As the disturbances propagate over the surface, they roll up into discrete eddies and leave a trail of dye stretching to the airfoil's surface.

The frames for $\omega t=20^{\circ}$ and $30^{\circ}$ show a single disturbance propagating down the surface, whereas the frame for $\omega t=40^{\circ}$ shows that the jet pulse is unable to overcome the adverse pressure gradient and is diverted away from the surface. This frame also shows that the flow is separating from the leading edge of the airfoil. This separated flow condition persists through $\omega t=135^{\circ}$ where the photographs show the boundary layer beginning to re-form. The boundary layer has completely re-formed by $\omega t=180^{\circ}$.

Figure 46 offers a comparison of the loads with no blowing, steady blowing, and pulsed blowing at $F^{+}=0.9$. Each blowing type has the same nominal value of $C_{\mu}$. In both cases blowing reduces the initial effects of trailing-edge separation. The distortion in the lift curve, caused by the formation and movement of the dynamic-stall vortex, is smaller in the steadyblowing case; it nearly disappears for pulsed blowing at $F^{+}=0.9$. The increase in the maximum lift coefficient is negligible for both pulsed and steady blowing. Hysteresis reduction occurs for each type of blowing, but pulsed blowing at $F^{+}=0.9$ is the most effective. The boundary layer re-forms approximately $4^{\circ}$ earlier with steady blowing and $5^{\circ}$ degrees earlier with pulsed blowing.

\section{Pulsed Blowing with $k=0.15$}

Figures 47-50 show the results of pulsed blowing for a constant momentum coefficient $\left(C_{\mu}=0.024\right.$ nominal) and a fixed reduced frequency $(k=0.15)$. The pulsed-blowing results are compared in figure 51 . The figure demonstrates that both steady and pulsed blowing have similar effects at $k=0.15$. Pulsed blowing at $F^{+}=0.9$ still shows a slight advantage in terms of hysteresis reduction and lift augmentation. Figure 52 offers a comparison of the loads with no blowing, steady blowing, and pulsed blowing at $F^{+}=0.9$. Each blowing type has the same nominal value of $C_{\mu}$, and in both cases blowing shows very little improvement at this reduced frequency. The only noticeable improvements are the small reductions in the initial effects of flow separation, a very small increase in the 
maximum lift, and small reductions in lift hysteresis during the downstroke portion of the cycles.

\section{Summary of Pulsed-Blowing Results}

Pulsed blowing and steady blowing cause virtually identical changes in the load behavior of the VR-7 for similar conditions. However, pulsed blowing creates substantial unsteadiness in the loads that is most pronounced in the post-stall region. This is actually a promising result because it suggests that the disturbances may be generated with relatively little energy and then amplified in the shear layer in accordance with the reasoning given in reference 12 . In all of the figures, it is apparent that pulsed blowing at $F^{+}=0.9$ has a slight advantage in terms of hysteresis reduction and lift augmentation. Unfortunately, the benefits are marginal at all but the quasi-steady reduced frequency. The lift-augmentation results at the quasisteady reduced frequency show general agreement with the results of references 12 and 13 .

\section{CONCLUSIONS}

1. Steady blowing can significantly enhance the lift produced by an airfoil undergoing a sinusoidalpitching motion by trapping a separation bubble at the leading edge during the upstroke portion of the cycle. When a separation bubble can be trapped at the leading edge, moment stall and lift stall are eliminated. Even when upper-surface blowing is not strong enough to maintain the separation bubble for the entire cycle, a benefit is still realized through a substantial reduction of the lift hysteresis. Increases in lift due to blowing are always greater than $C_{\mu}$ (non-dimensional jet momentum).

2. Blowing can reduce the severity (or even completely eliminate the presence) of fluctuations in the loads caused by flow reversal and the formation and initial movement of the dynamic-stall vortex.

3. Steady upper-surface blowing creates a significant thrust force for all conditions tested in this study. Thrust magnitudes are always less than $C_{\mu}$ because of wall friction.

4. The best steady-blowing results were obtained at the lowest reduced frequency tested, the lowest mean angle of attack, and the highest value of $C_{\mu}$. The beneficial changes to the unsteady loads that are derived from upper-surface blowing are countered by increases in either the reduced frequency or the mean angle of oscillation.

5. The benefits from pulsed blowing at the quarter-chord location are marginal at all but the lowest reduced frequency (quasi steady).

6. The lift-augmentation results for pulsed blowing at the lowest reduced frequency (quasi steady) show general agreement with the results of references 12 and 13.

7. Pulsed blowing and steady blowing cause virtually identical changes in the unsteady load behavior of the VR-7 for similar conditions. Pulsed blowing at $F^{+}=0.9$ has a slight advantage in terms of hysteresis reduction and lift augmentation.

\section{REFERENCES}

1. McCroskey, W. J.; McAlister, K. W.; Carr, L. W.; Pucci, S. L.; Lambert, O.; and Indergand, R. F.: Dynamic Stall on Advanced Airfoil Sections. J. American Helicopter Soc., vol. 26, no. 3, July 1981, pp. 40-50.

2. Prouty, R. W.: Helicopter Performance, Stability, and Control. PWS Engineering, 1986.

3. Ham, N. D.; and Young, M. I.: Torsional Oscillation of Helicopter Blades Due to Stall. J. Aircraft, vol. 3, no. 3, 1966, pp. 218-224.

4. Carr, L. W.; McAlister, K. W.; and McCroskey, W. J.: Analysis of the Development of Dynamic Stall Based on Oscillating Airfoil Experiments. NASA TN D-8382, 1977.

5. McCroskey, W. J.: The Phenomenon of Dynamic Stall. NASA TM-81264, 1981.

6. Ham, N. D.: Aerodynamic Loading on a TwoDimensional Airfoil During Dynamic Stall. AIAA J., vol. 6, no. 10, 1968, pp. 1927-1934.

7. Yu, Y. H.; Lee, S.; McAlister, K. W.; Tung, C.; and Wang, C. M.: High Lift Concepts for Rotorcraft Applications. The 49th American Helicopter Society Annual Forum, St. Louis, Mo., May 19-21, 1993, pp. 1141-1166.

8. McAlister, K. W.; and Tung, C.: Suppression of Dynamic Stall with a Leading-Edge Slat on a VR-7 Airfoil. NASA TP-3357, 1993. 
9. Plantin de Hugues, P.; McAlister, K. W.; and Tung, C.: Effect of an Extendable Slat on the Stall Behavior of a VR-12 Airfoil. NASA TP-3407, 1993.

10. Lee, S.; McAlister, K. W.; and Tung, C.: Characteristics of a Deformable Leading Edge for High Performance Helicopter Rotor. AIAA Paper 93-3526, 11th Applied Aerodynamics Conference, Monterey, Calif., Aug. 9-11, 1993.

11. Seifert, A.; Bachar, T.; and Wygnanski, I.: Oscillatory Blowing, A Tool to Delay Boundary Layer Separation. AIAA Paper 93-0440, 31st Aerospace Sciences Meeting \& Exhibit, Reno, Nev., Jan. 11-14, 1993.
12. Seifert, A.; Daraby, A.; Nishri, B.; and Wygnanski, I.: The Effects of Forced Oscillations on the Performance of Airfoils. AIAA Paper 93-3264, AIAA Shear Flow Conference, Orlando, Fla., July 6-9, 1993.

13. Lovato, J. A.; and Troutt, T. R.: Active Control of the Shear Layer on a Static Airfoil. AIAA Paper 93-0442, 31 st Aerospace Sciences Meeting \& Exhibit, Reno, Nev., Jan. 11-14, 1993. 

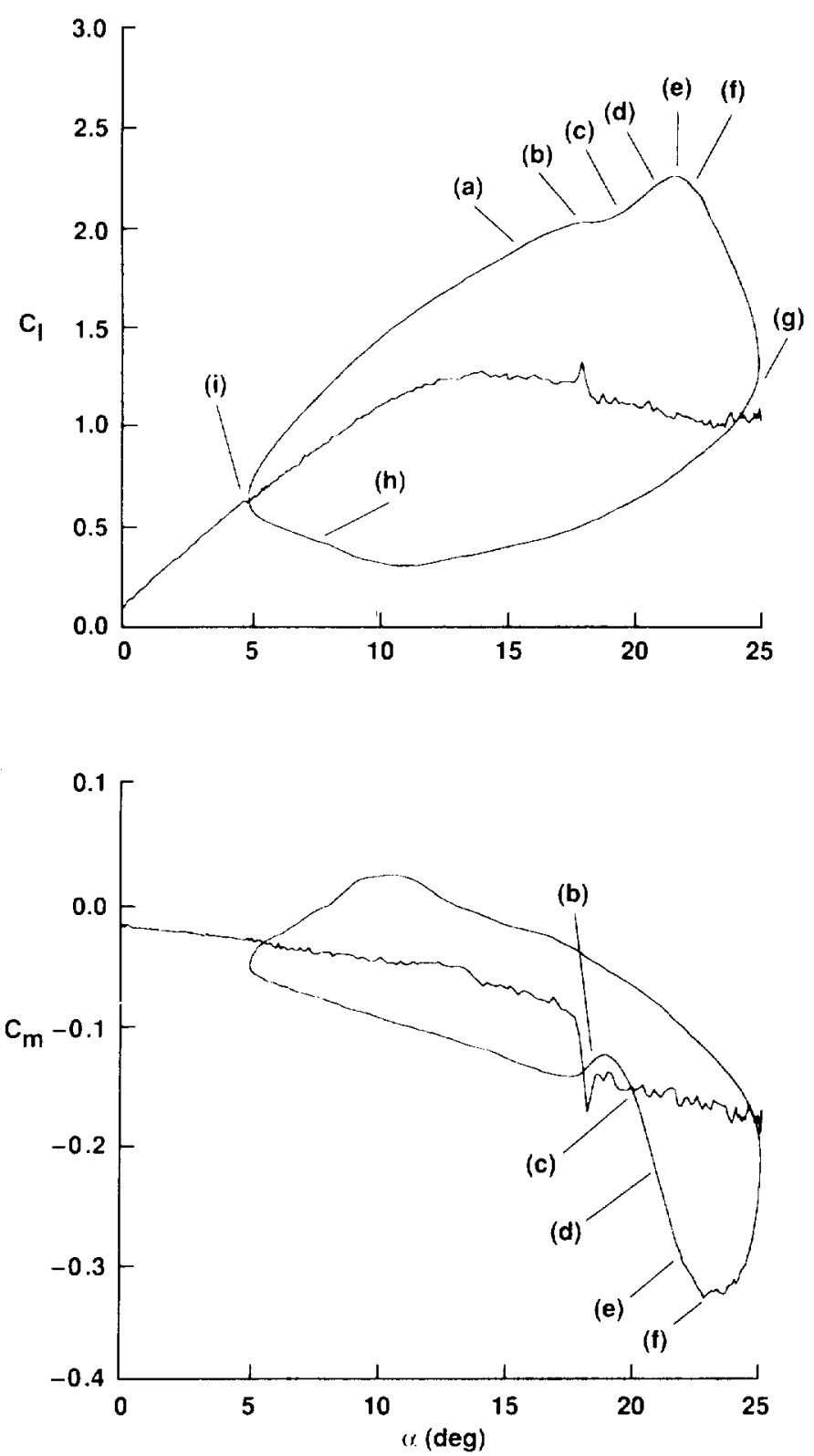

(a) quasi-steady stall angle exceeded

(b) thin layer of reversed flow covers the surface and dynamic stall vortex begins to form

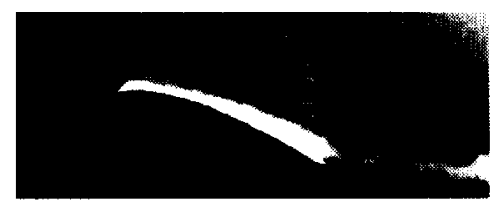

(c) moment stall begins and large eddies form in boundary layer

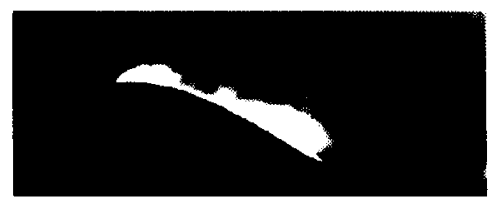

(d) stall vortex continues to gain strength and eddies in the boundary layer coalesce

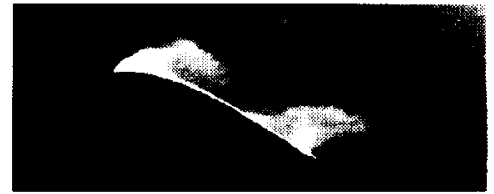

(e) lift stall begins

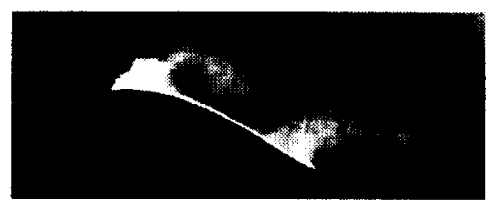

(f) maximum negative moment

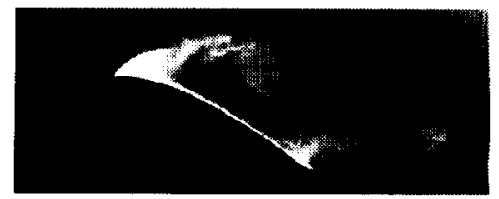

(g) full stall

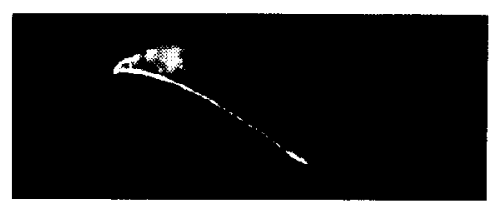

(h) boundary layer re-forms

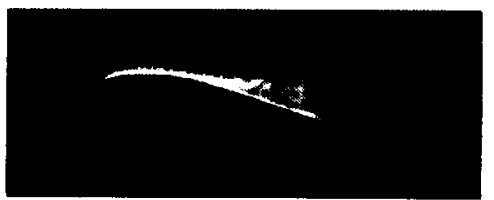

(i) return to unstalled values

Figure 1. Dynamic stall events on the VR-7 airfoil for $\alpha_{m}=15^{\circ}$ and $k=0.15$ at $R e^{\prime}=200,000$. 


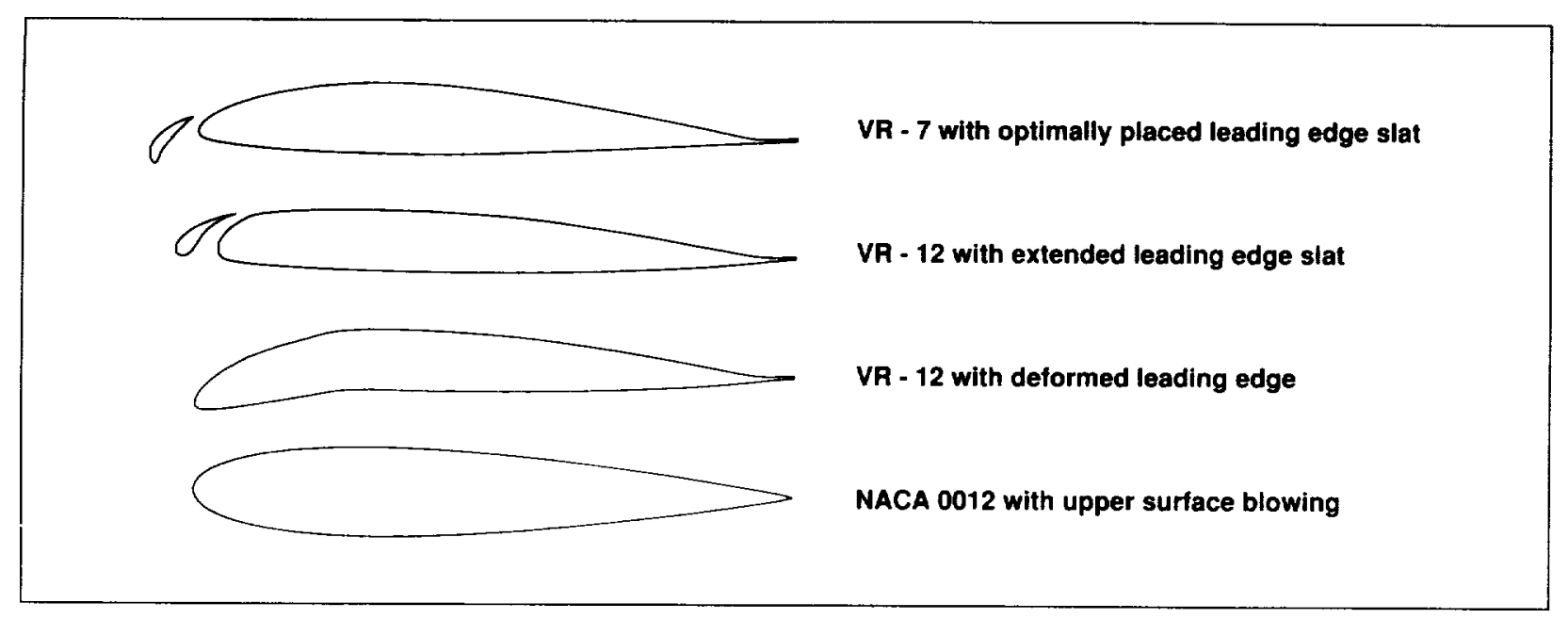

Figure 2. Summary of dynamic-stall control experiments performed at the U. S. Army Aeroflightdynamics Directorate Water Tunnel Facility.

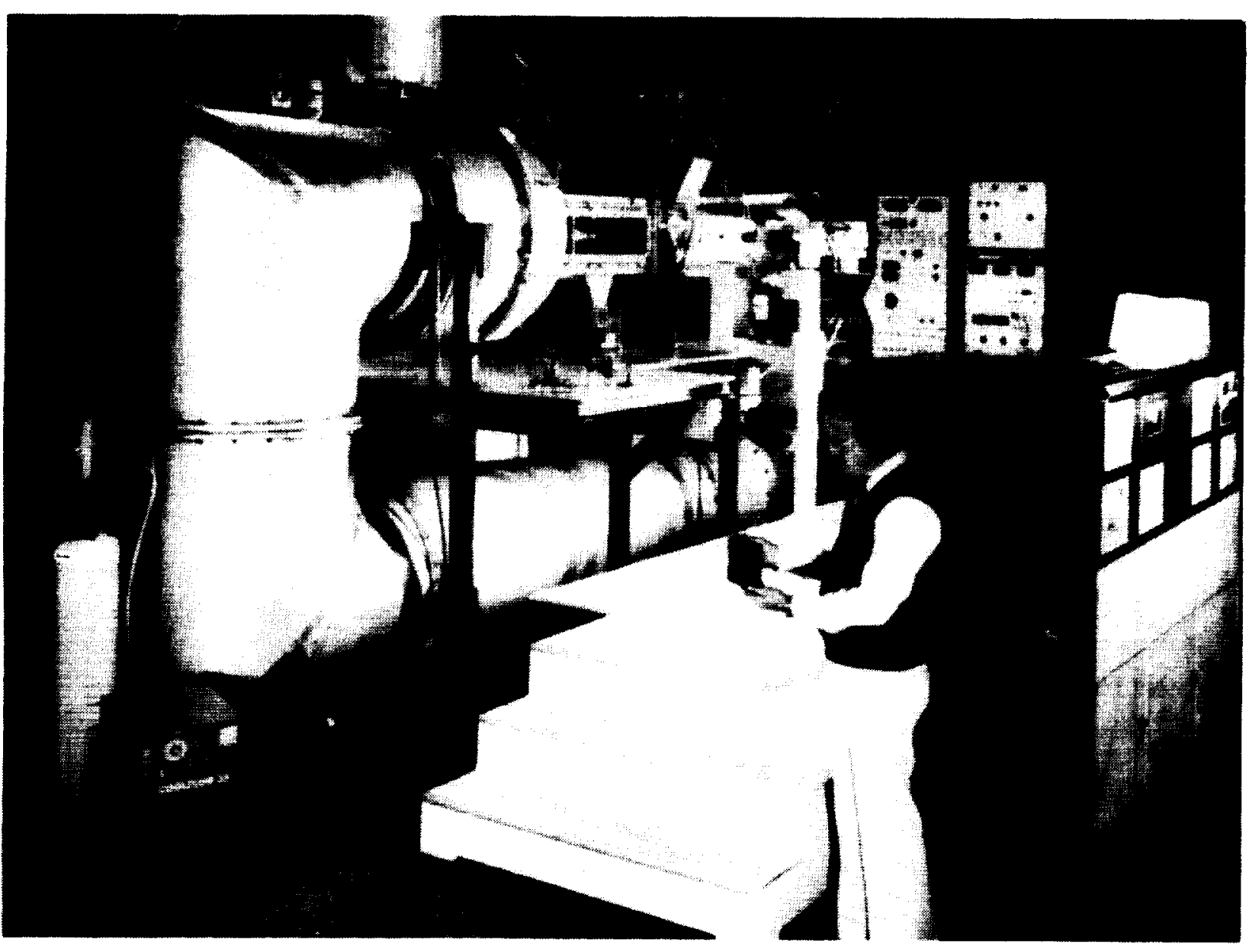

Figure 3. U.S. Army Aeroflightdynamics Directorate 8- by 12-Inch Water Tunnel. 


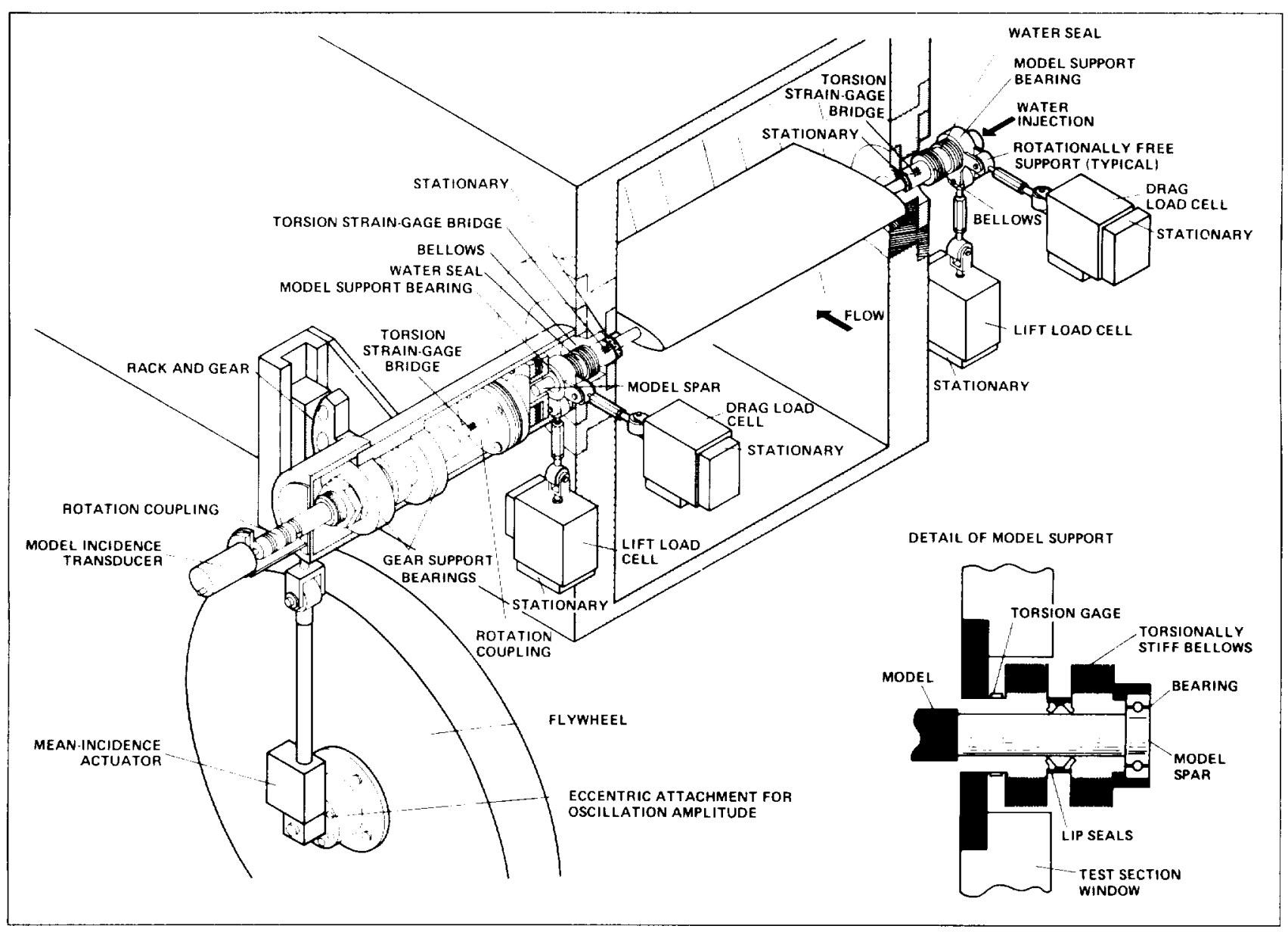

Figure 4. Model installation and force balance system.

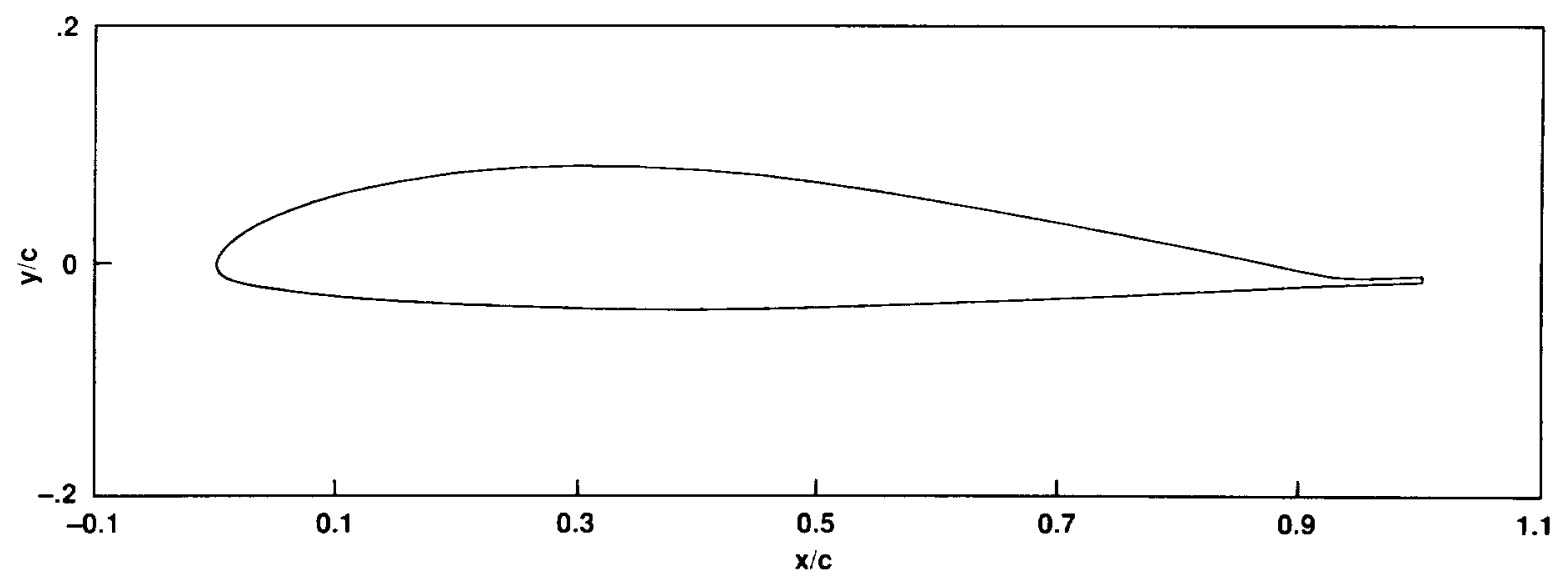

Figure 5. Boeing-Vertol VR-7 airfoil. 

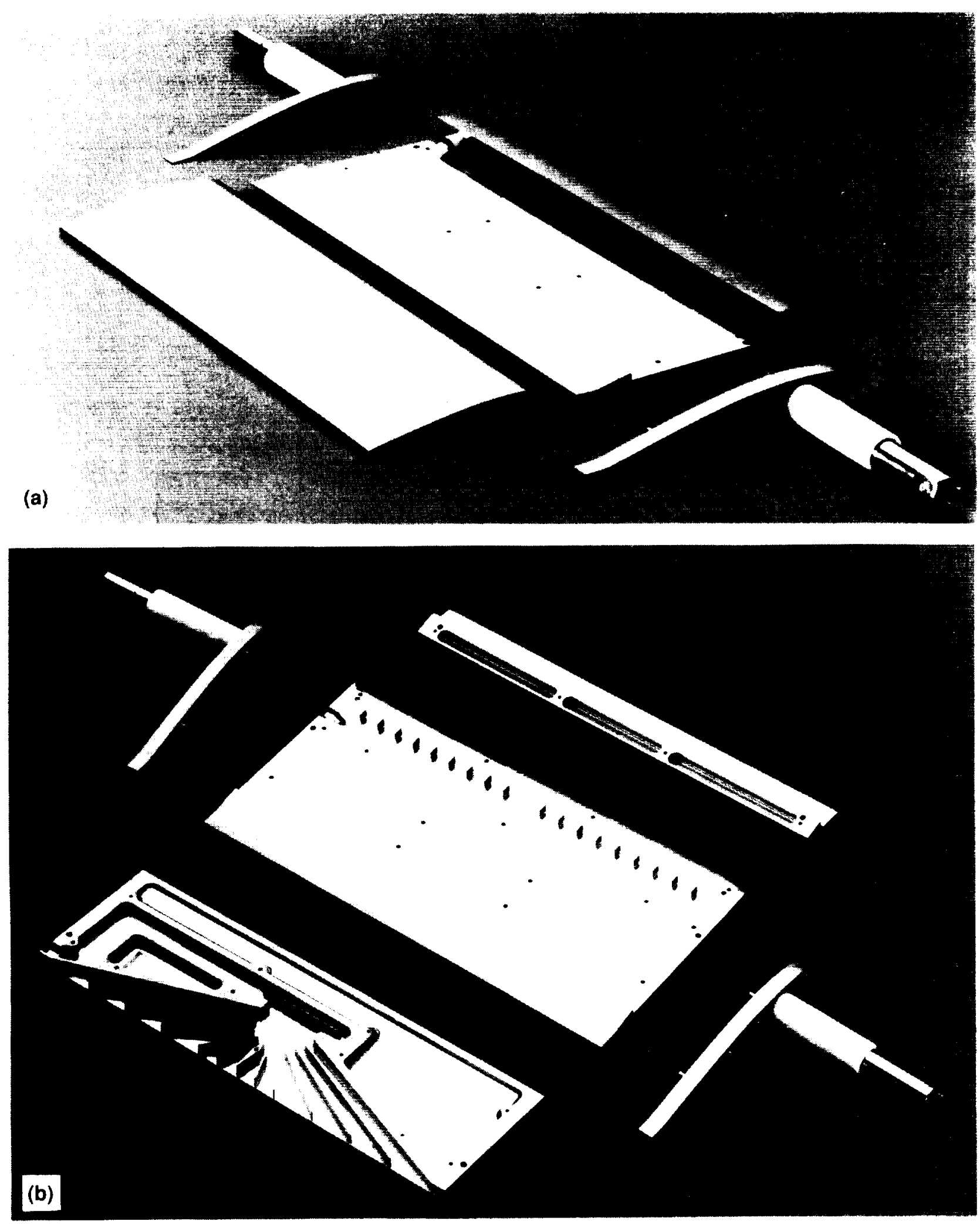

Figure 6. VR-7 model disassembled to reveal the internal diffuser and the slot. 


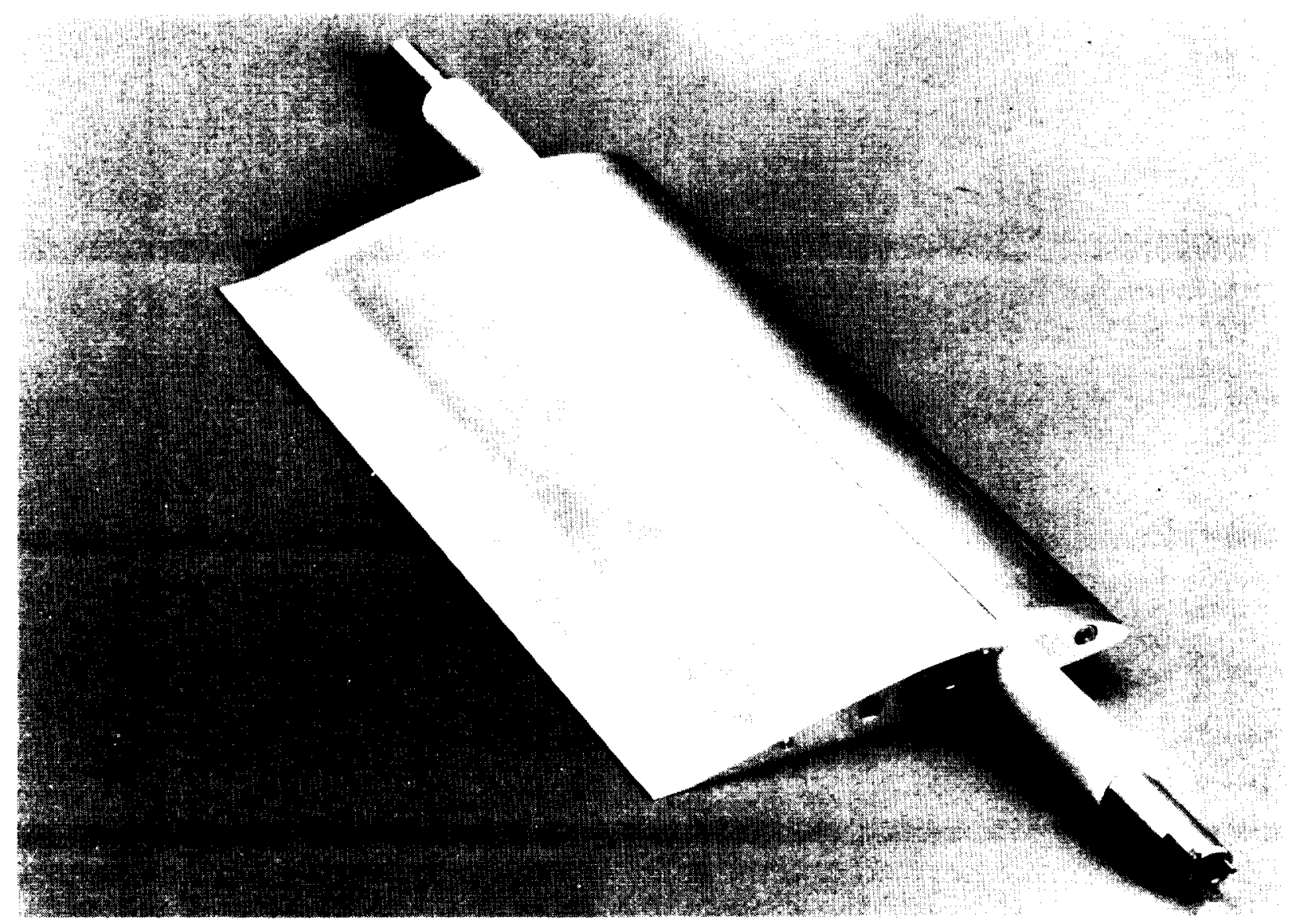

Figure 7. Assembled VR-7 model.

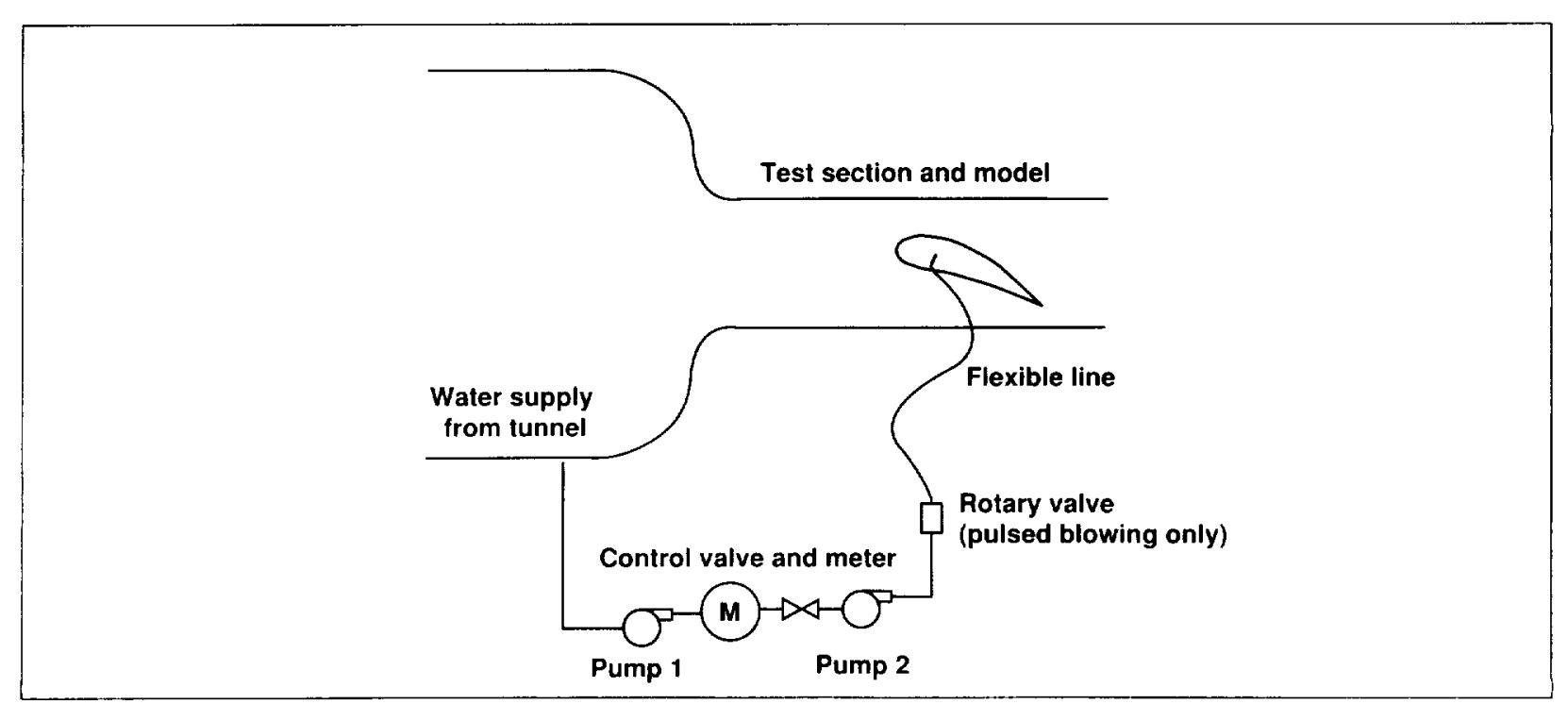

Figure 8. Schematic of blowing system. 

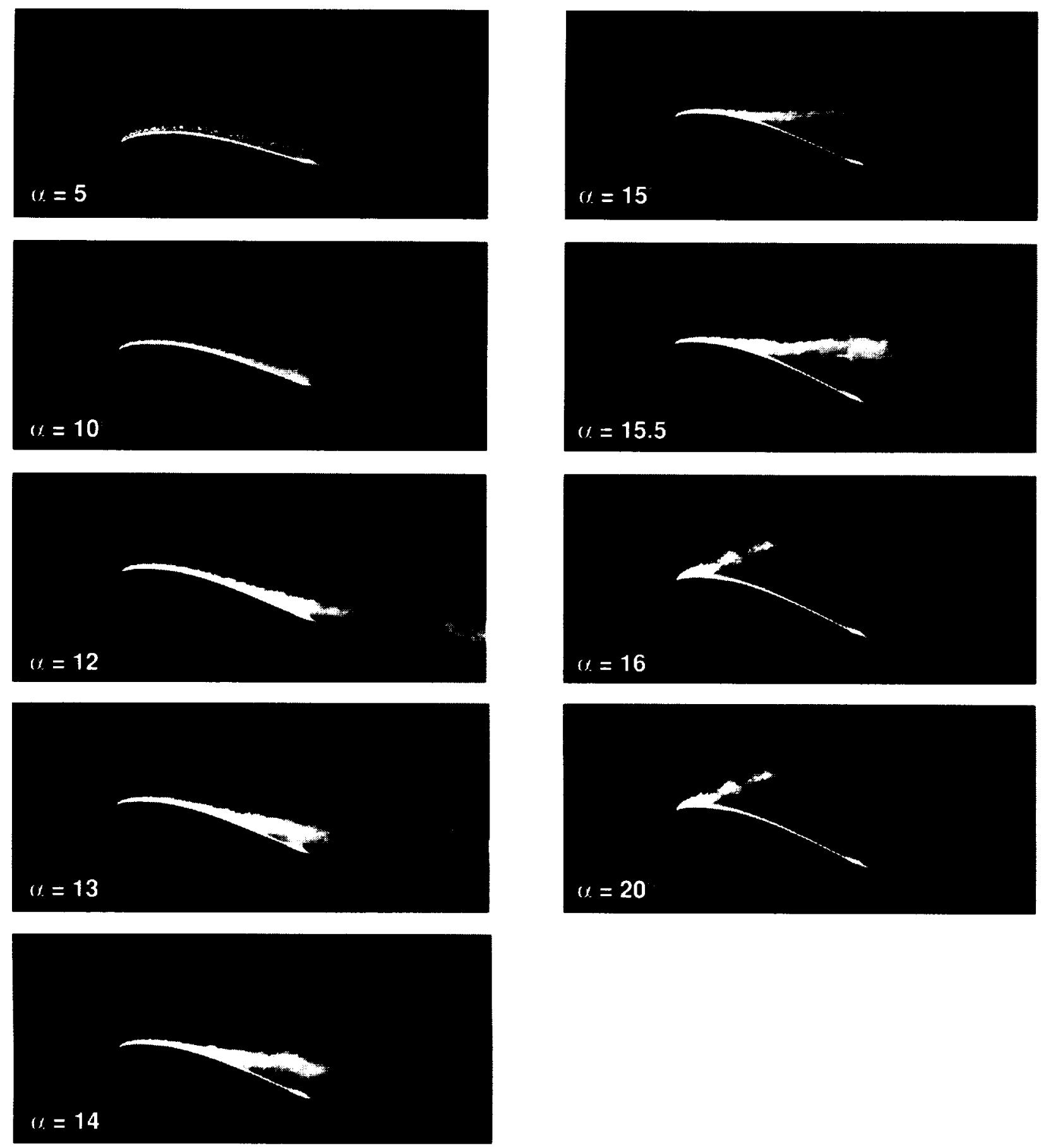

(a) Boundary layer and wake visualization.

Figure 9. Flow visualization and load measurements for $\alpha_{m}=10^{\circ}$ and $k=0.004$ with no blowing. 


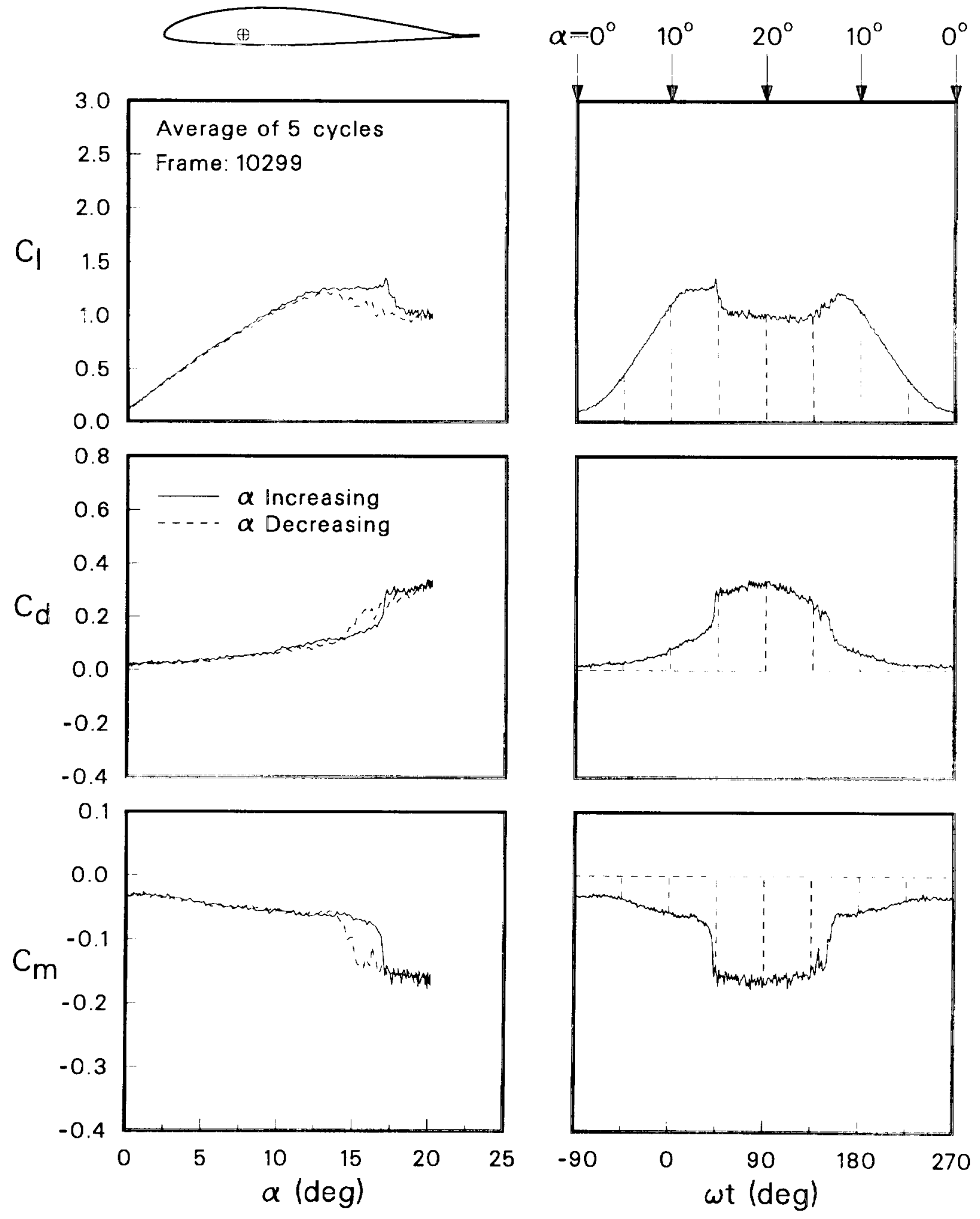

(b) Unsteady force and moment loads.

Figure 9. Concluded. 

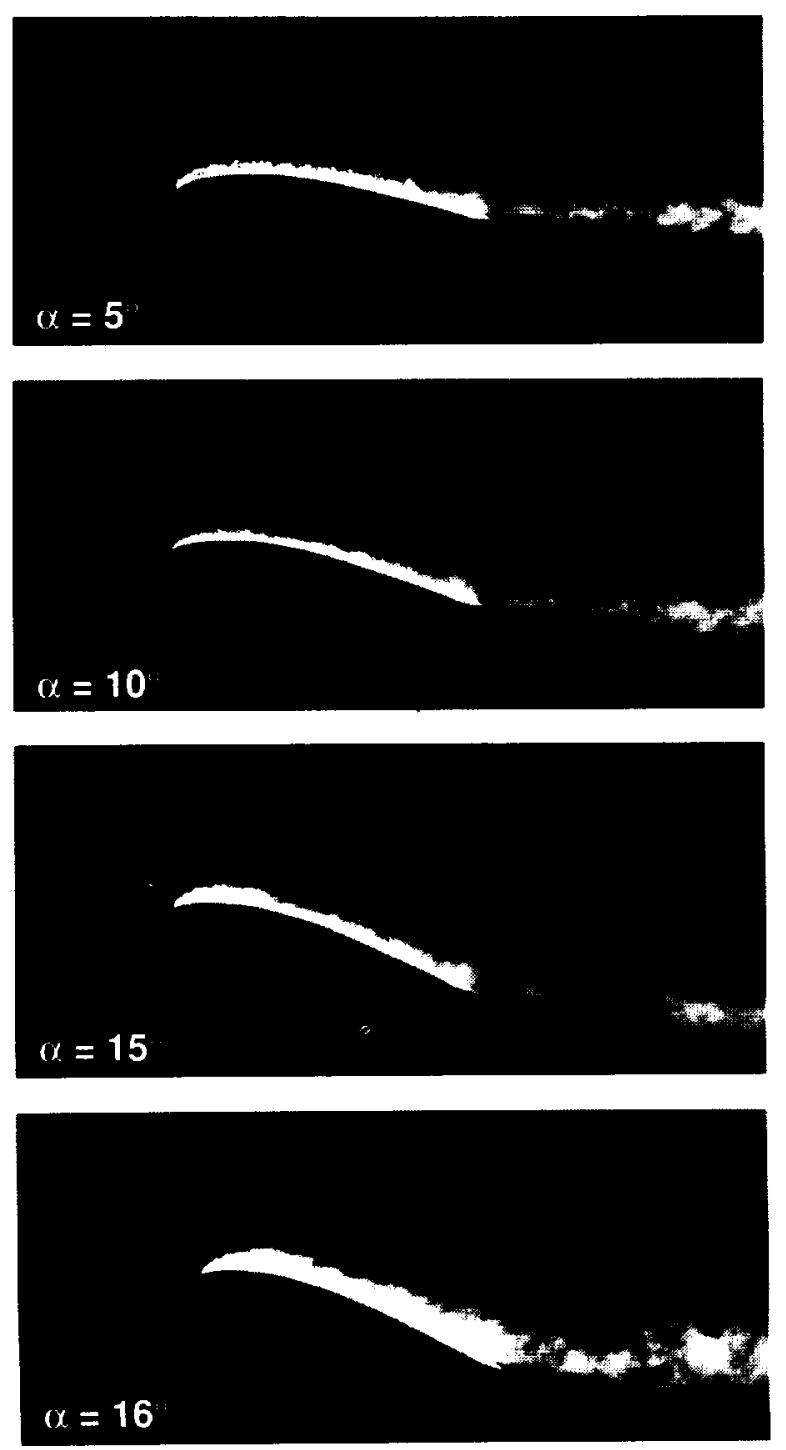

(a) Boundary layer and wake visualization.

Figure 10. Flow visualization and load measurements for $\alpha_{m}=10^{\circ}$ and $k=0.005$ with steady blowing at $C_{\mu}=0.16$.
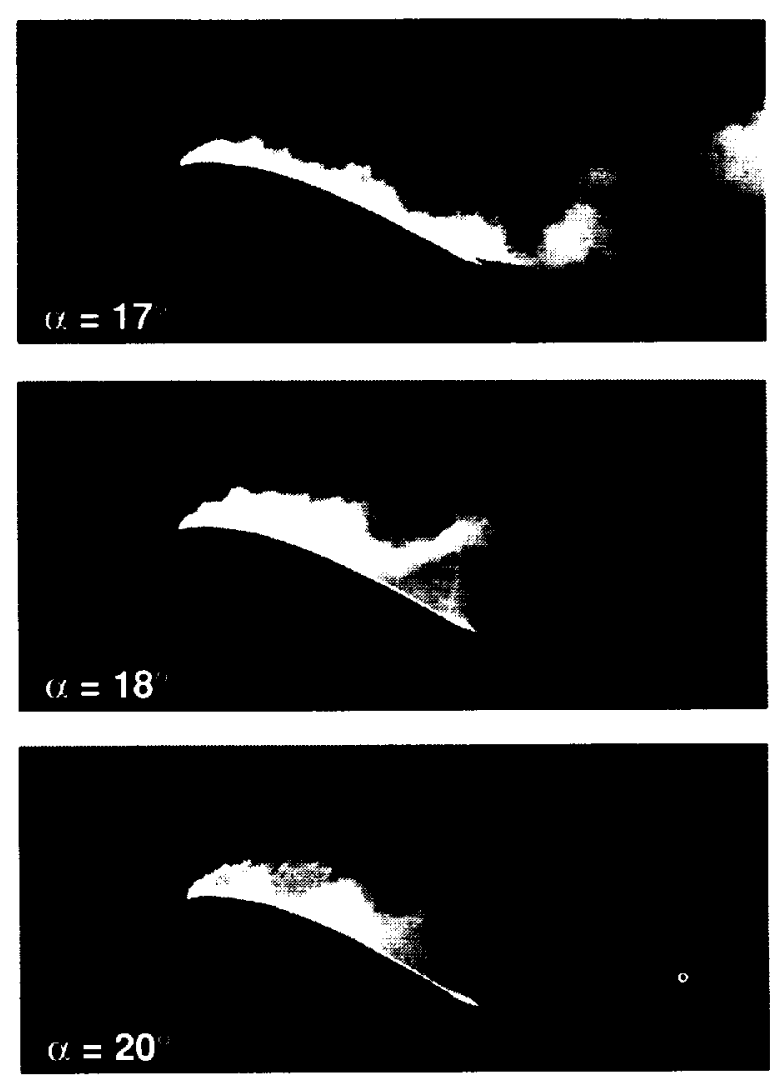

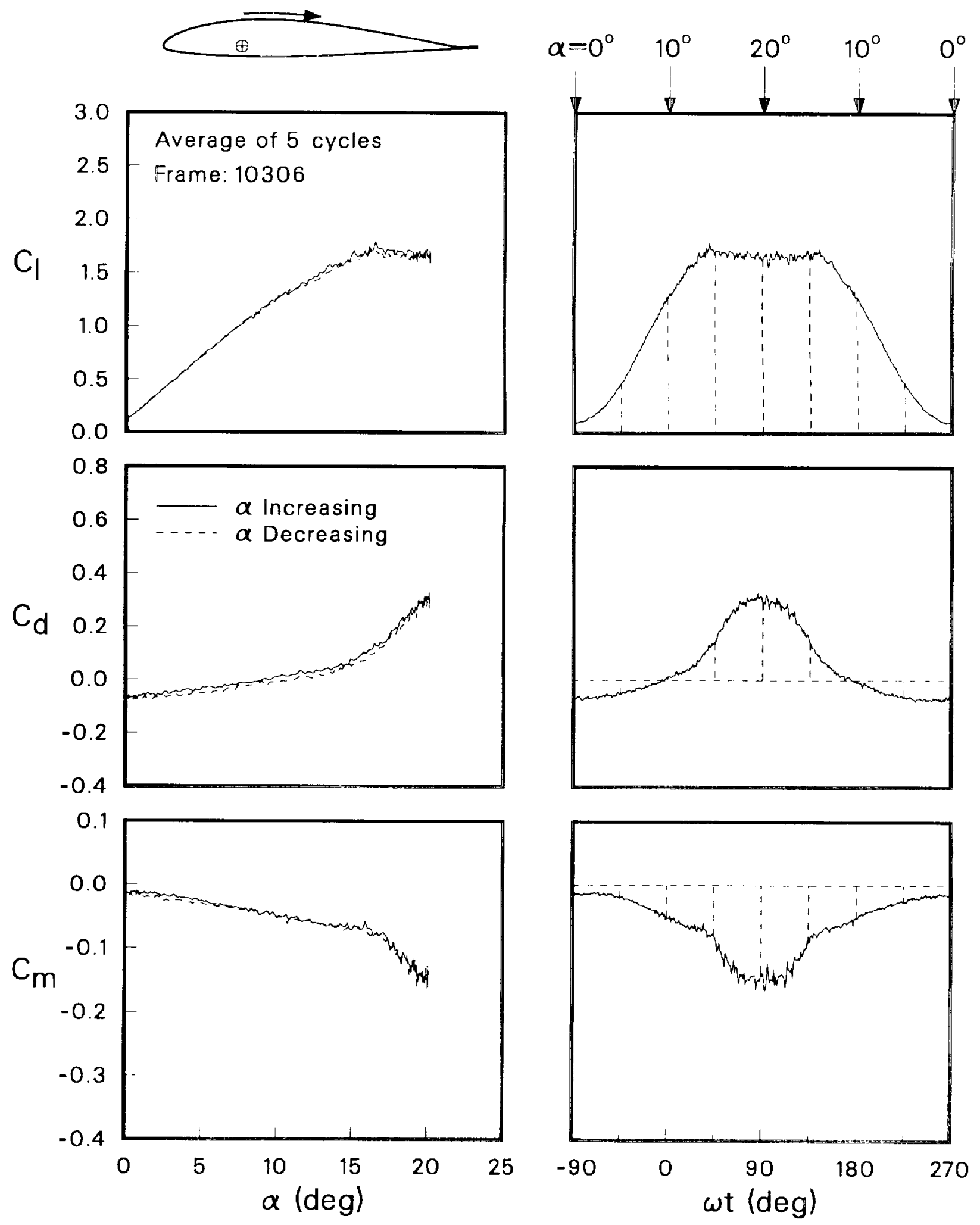

(b) Unsteady force and moment loads.

Figure 10. Concluded. 

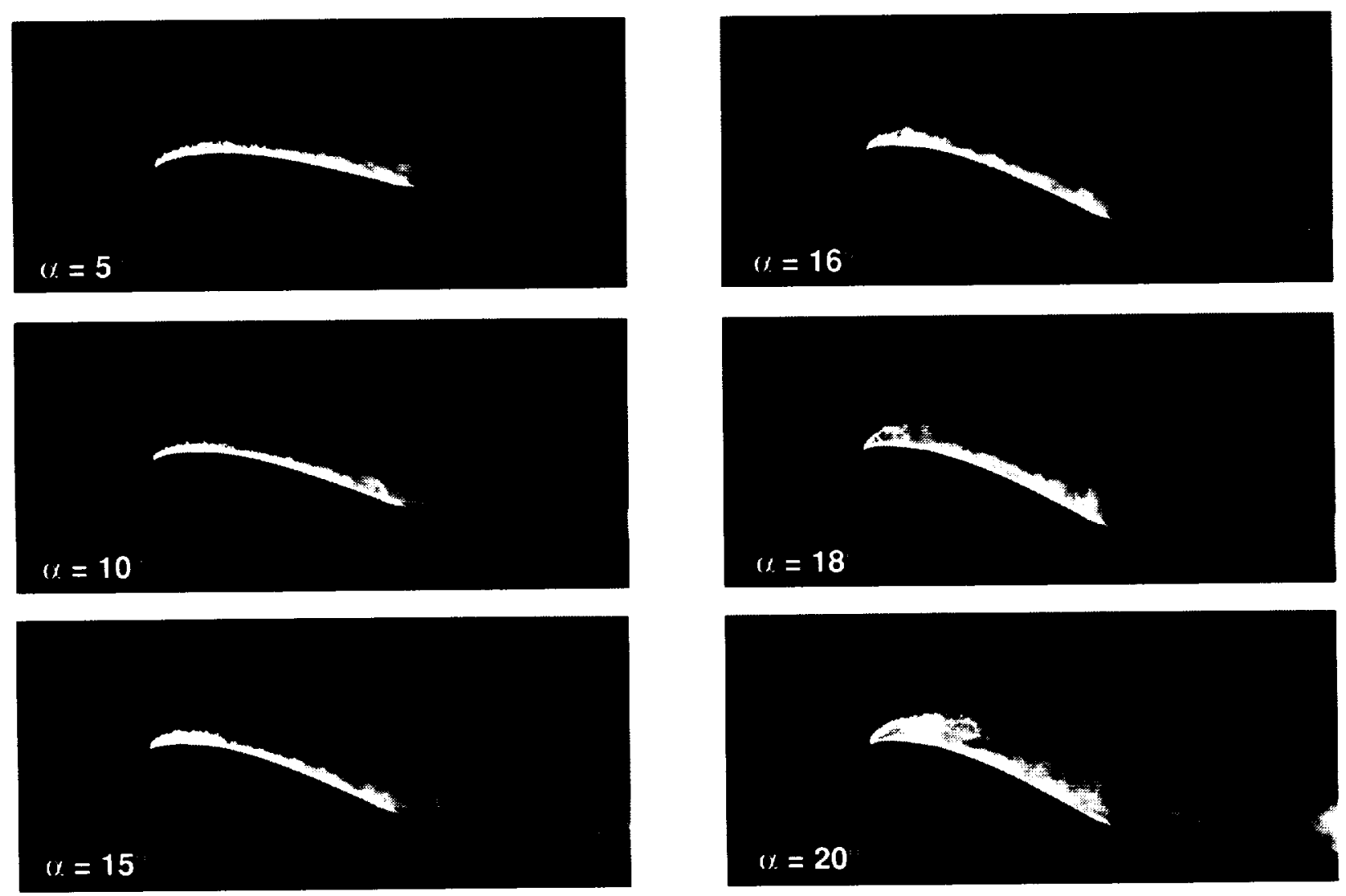

(a) Boundary layer and wake visualization.

Figure 11. Flow visualization and load measurements for $\alpha_{m}=10^{\circ}$ and $k=0.006$ with steady blowing at $\mathrm{C}_{\mu}=0.54$. 


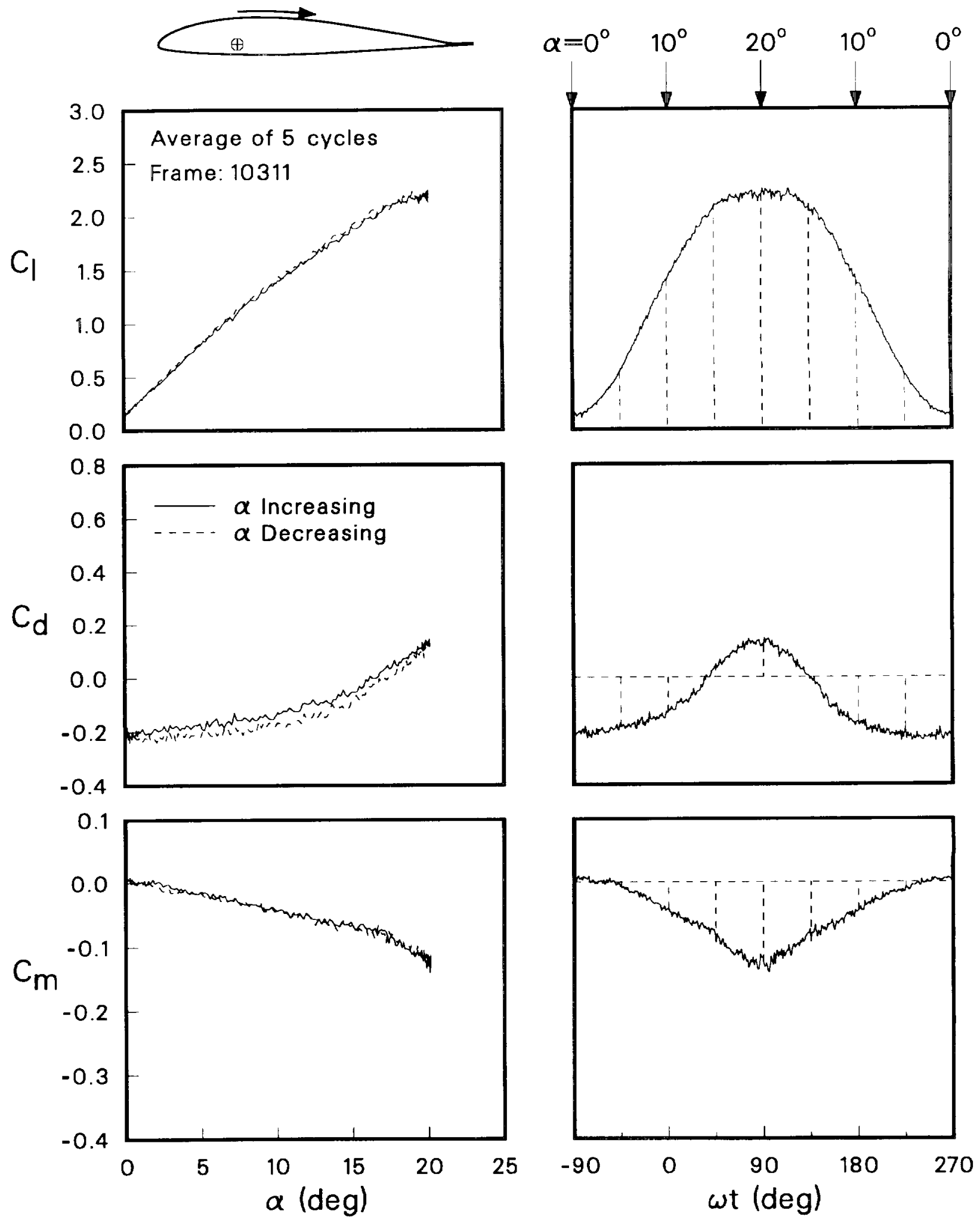

(b) Unsteady force and moment loads.

Figure 11. Concluded. 

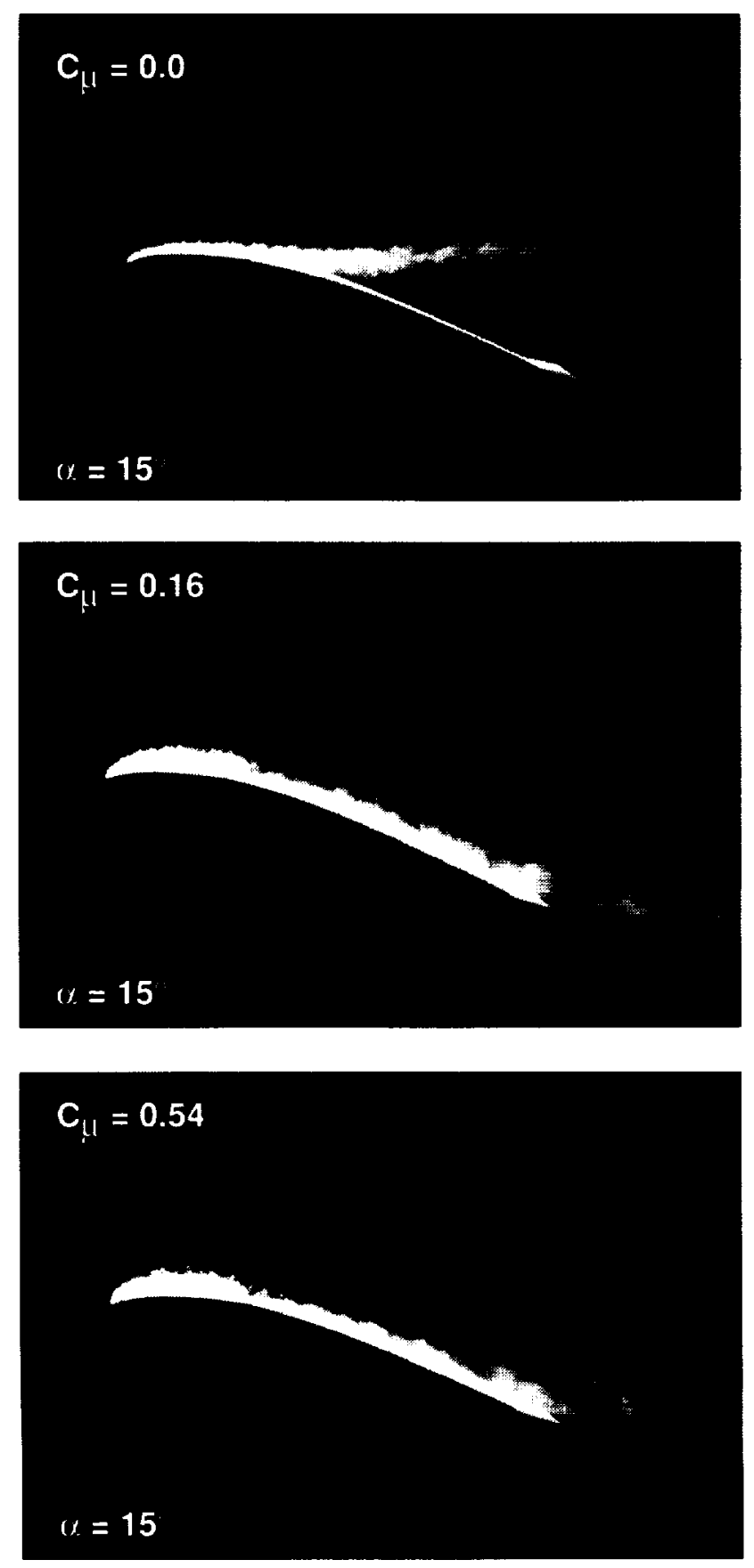

(a) Boundary layer and wake visualization.

Figure 12. Flow visualization and load measurements for $\alpha_{m}=10^{\circ}$ and $k=0.005$ (nominal) at $\alpha=15^{\circ}$ with different blowing rates. 


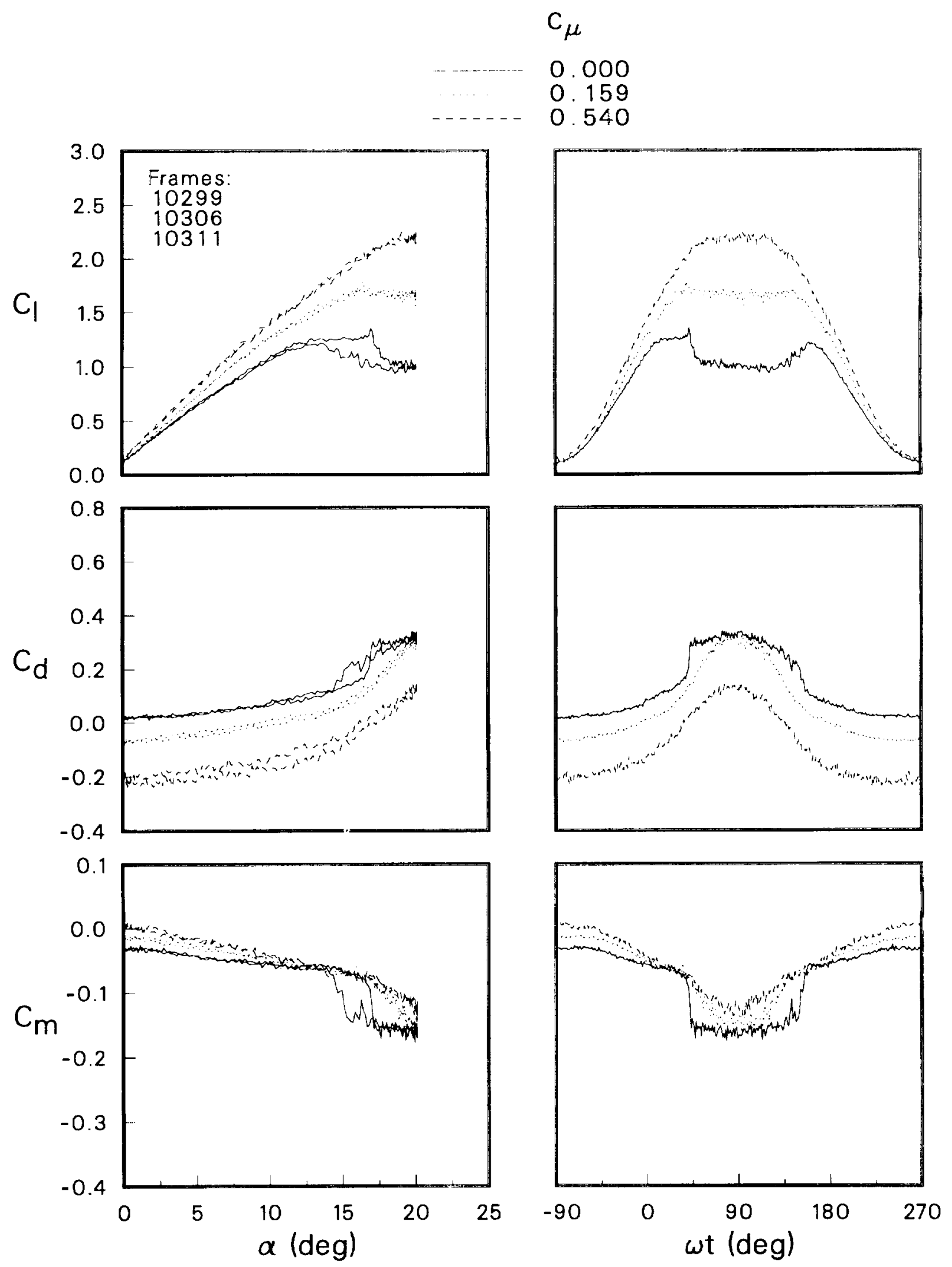

(b) Unsteady force and moment loads.

Figure 12. Concluded. 

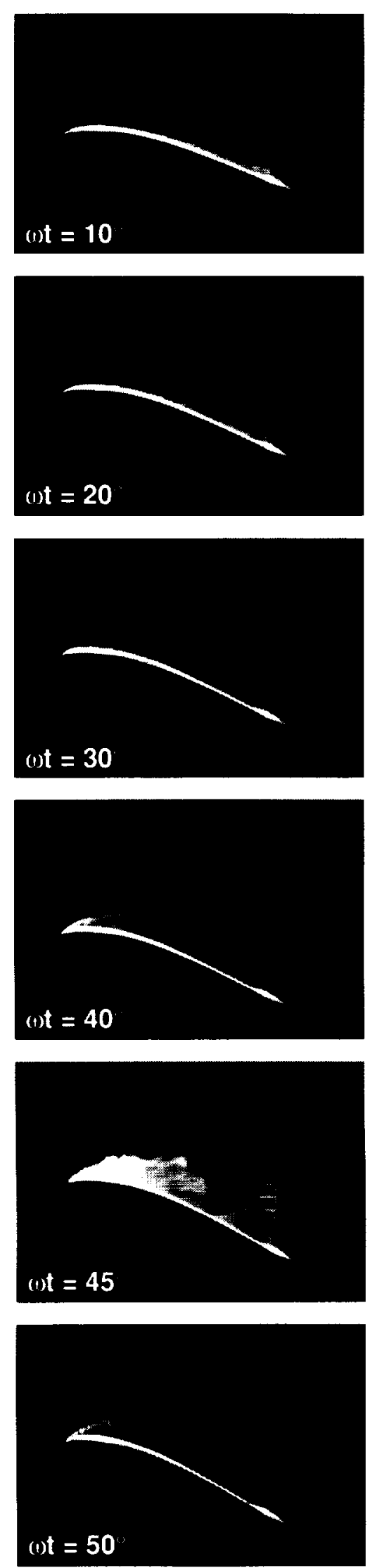
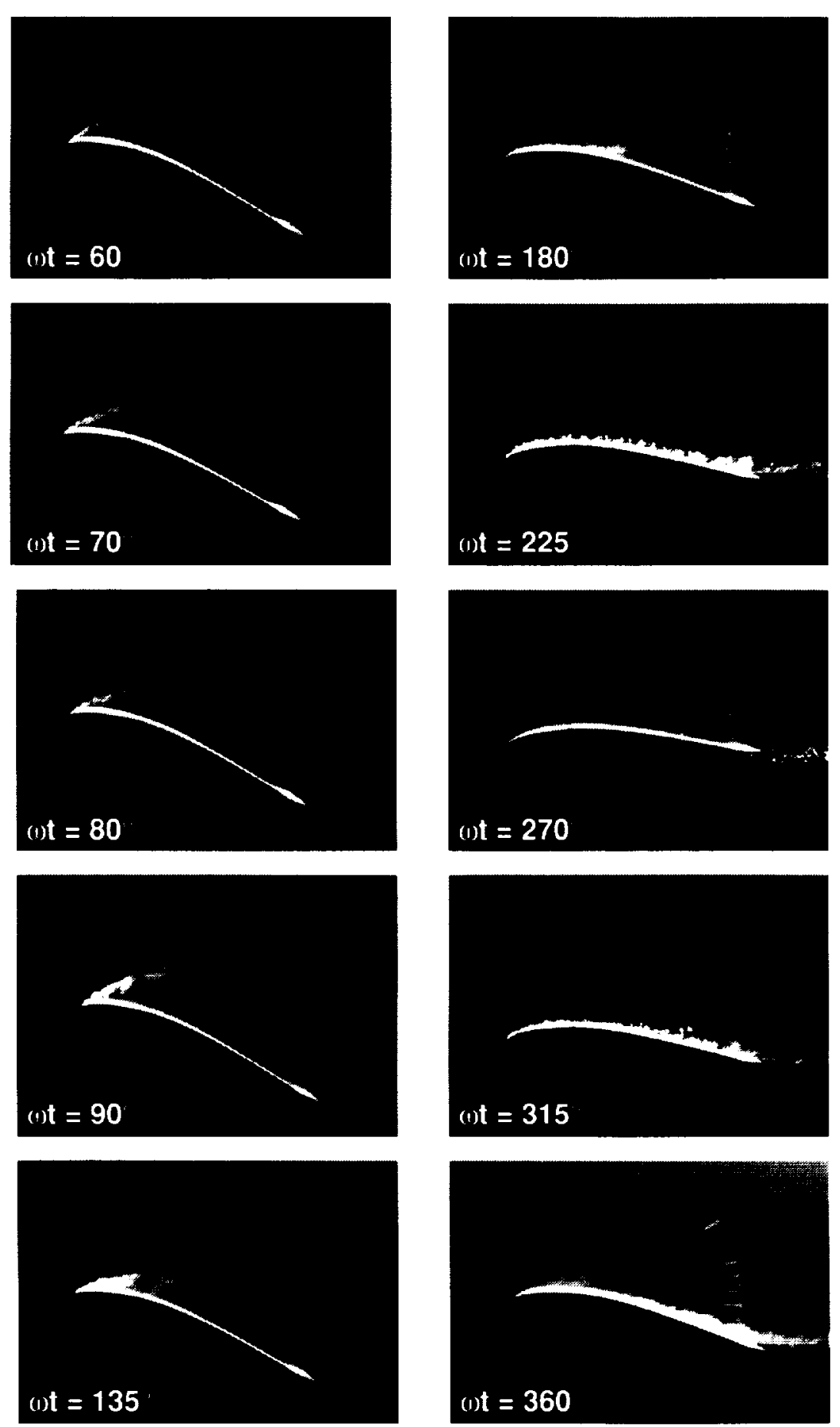

(a) Boundary layer and wake visualization.

Figure 13. Flow visualization and load measurements for $\alpha_{m}=10^{\circ}$ and $k=0.05$ with no blowing. 


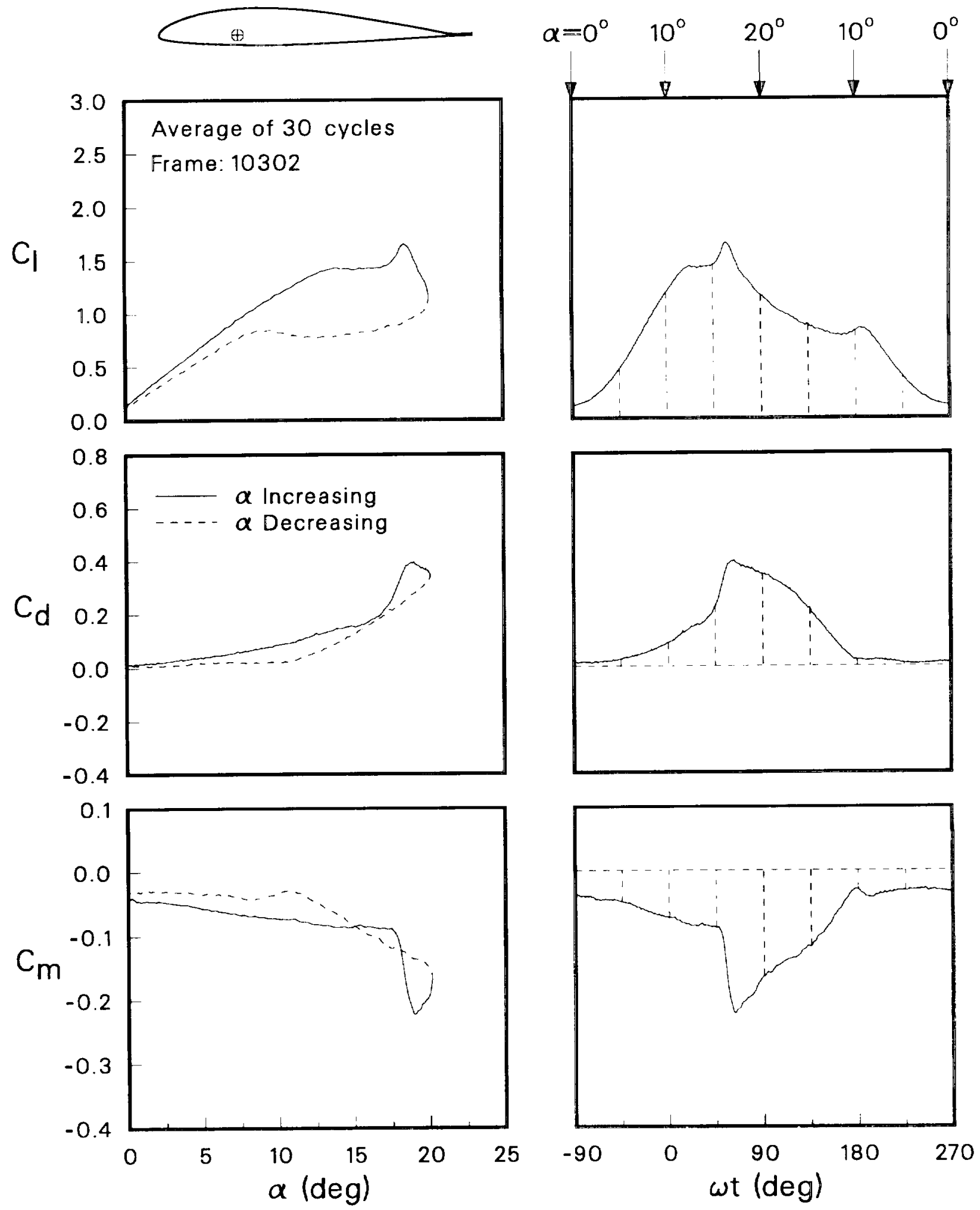

(b) Unsteady force and moment loads.

Figure 13. Concluded. 

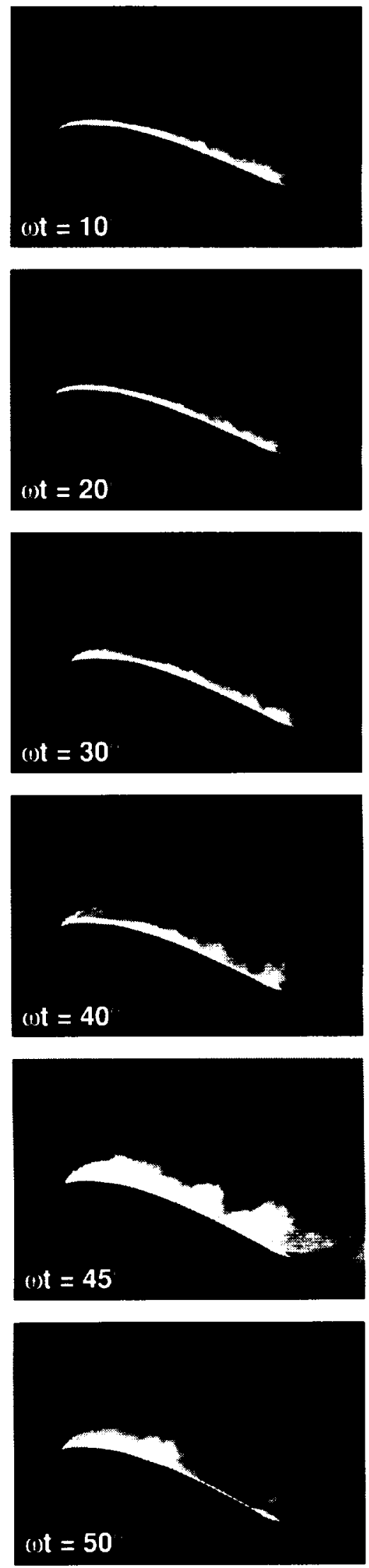
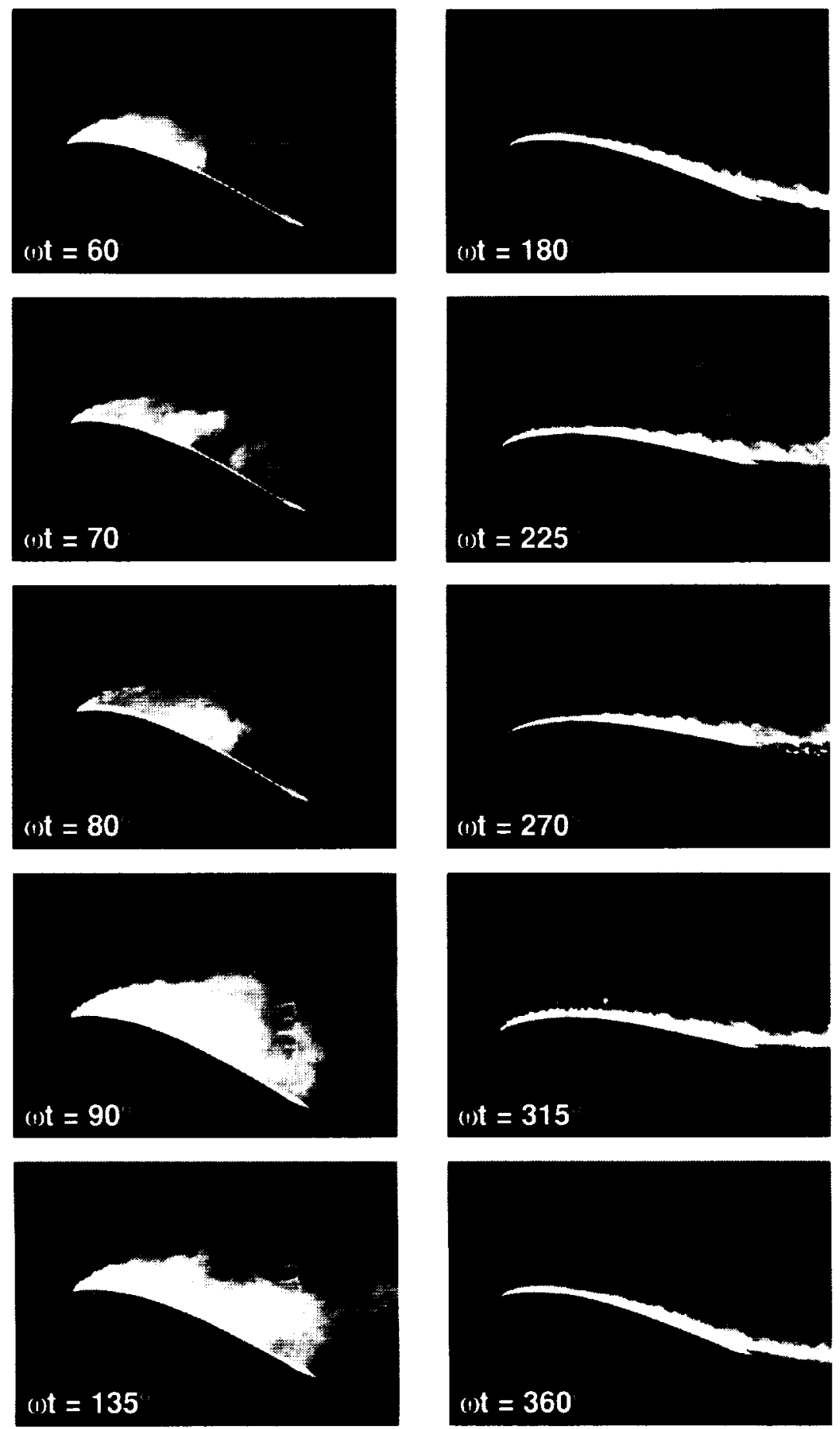

(a) Boundary layer and wake visualization.

Figure 14. Flow visualization and load measurements for $\alpha_{m}=10^{\circ}$ and $k=0.05$ with steady blowing at $C_{\mu}=0.16$. 


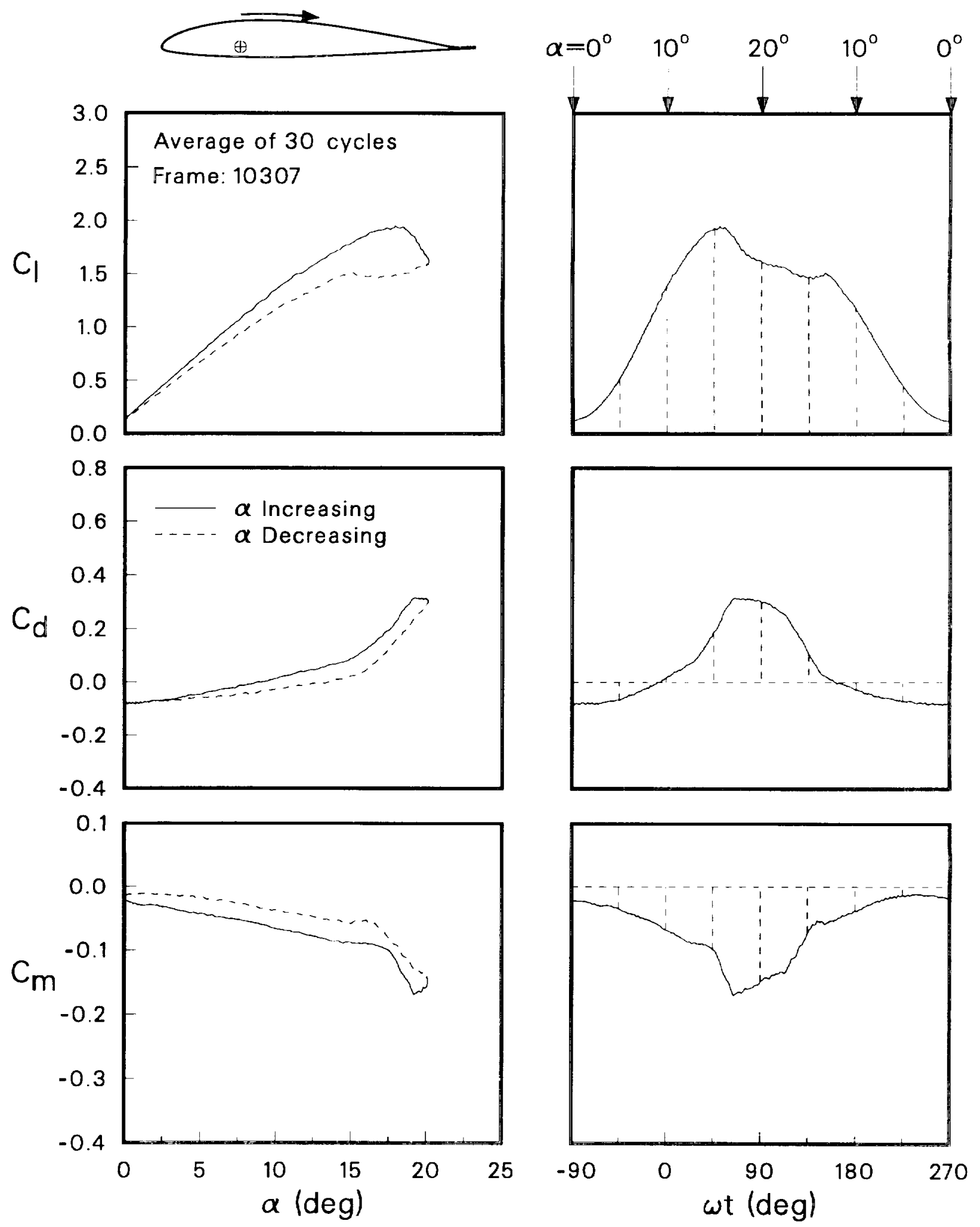

(b) Unsteady force and moment loads.

Figure 14. Concluded. 

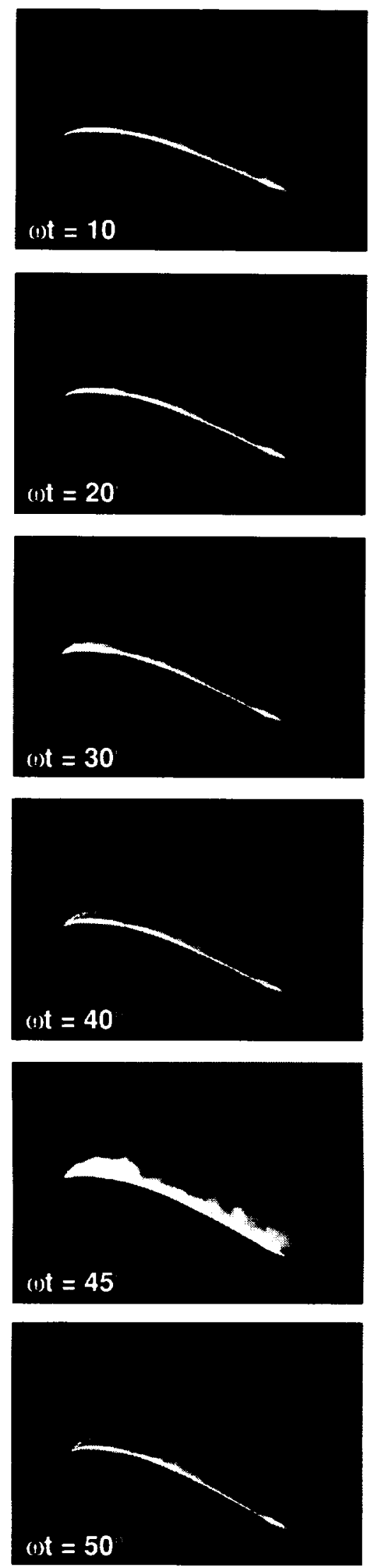
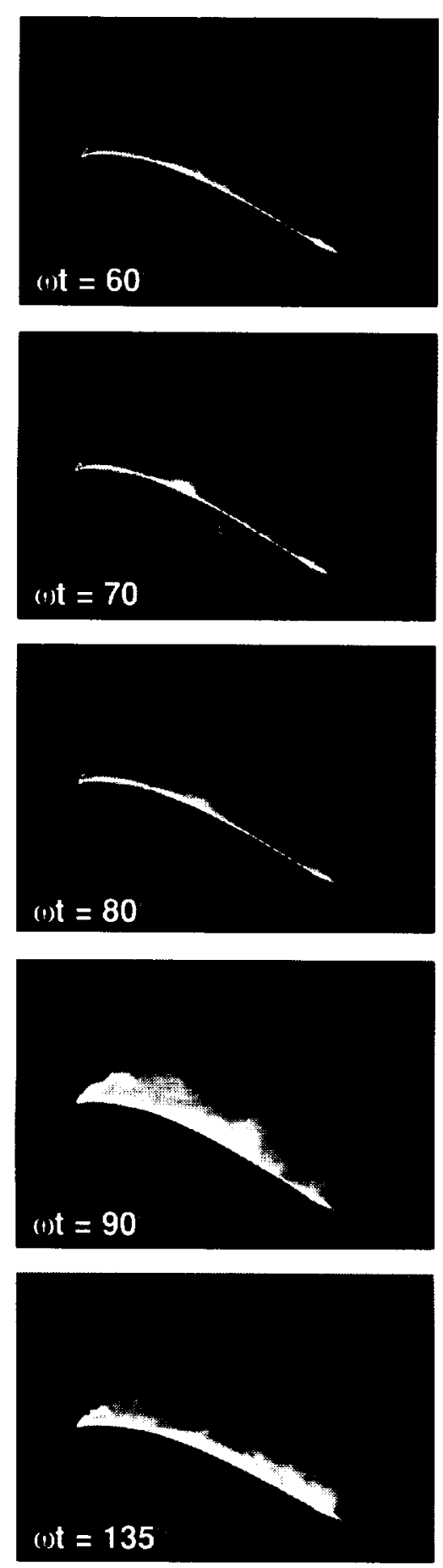

\section{(1) $t=180$}
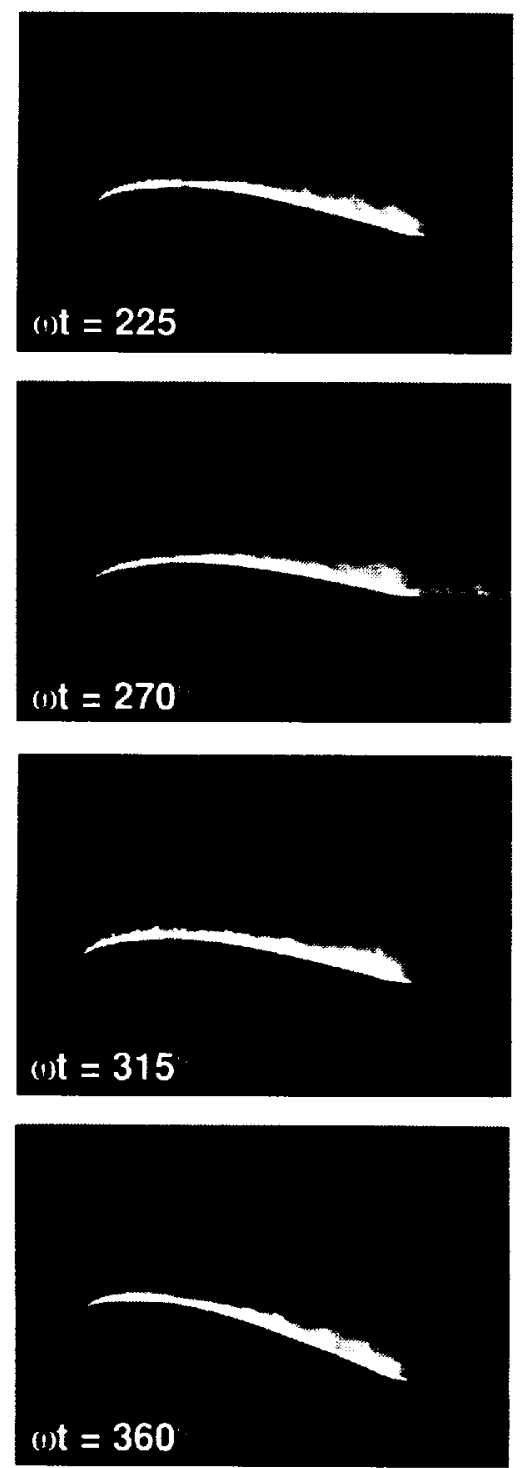

(a) Boundary layer and wake visualization.

Figure 15. Flow visualization and load measurements for $\alpha_{m}=10^{\circ}$ and $k=0.05$ with steady blowing at $C_{\mu}=0.57$. 


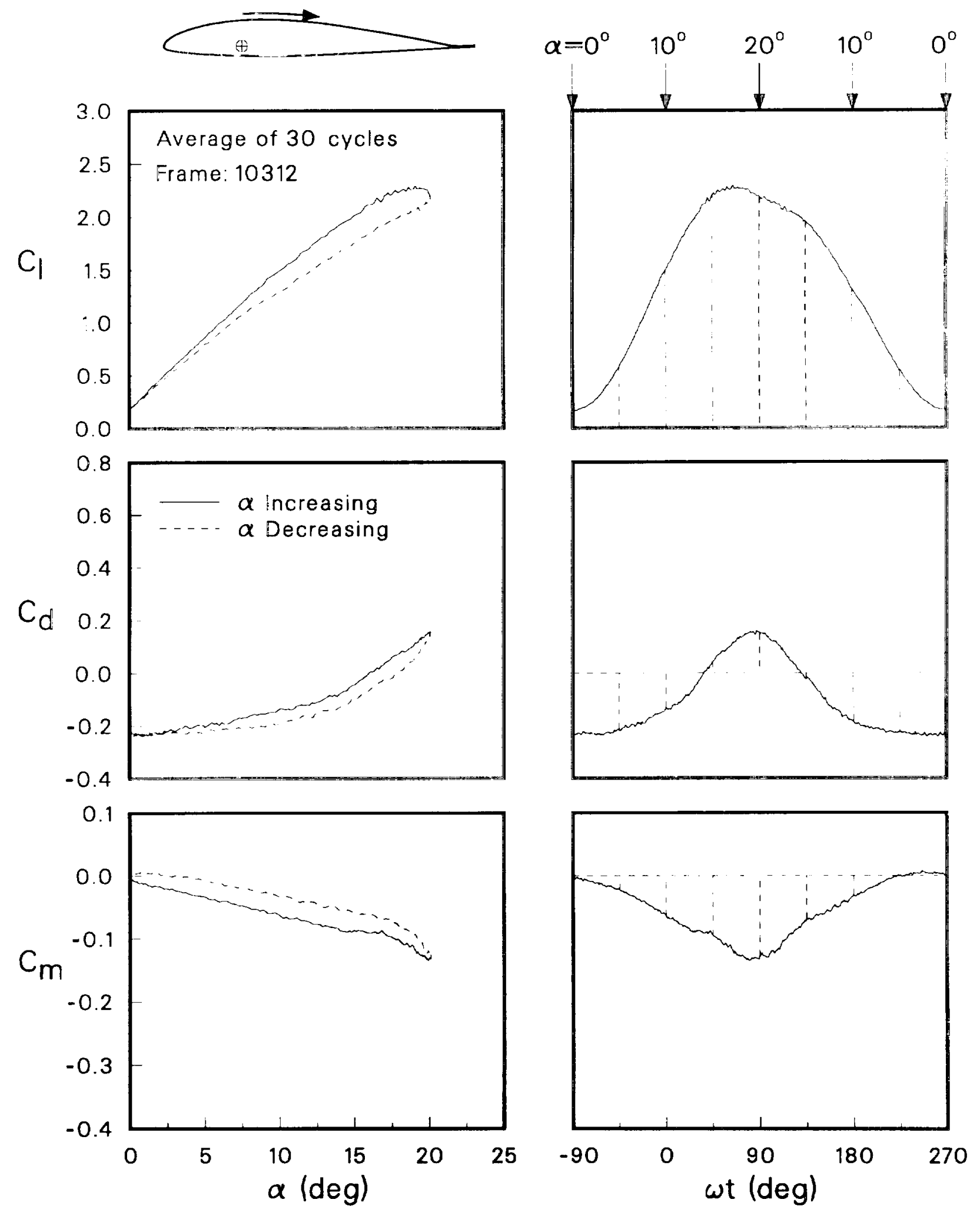

(b) Unsteady force and moment loads.

Figure 15. Concluded. 

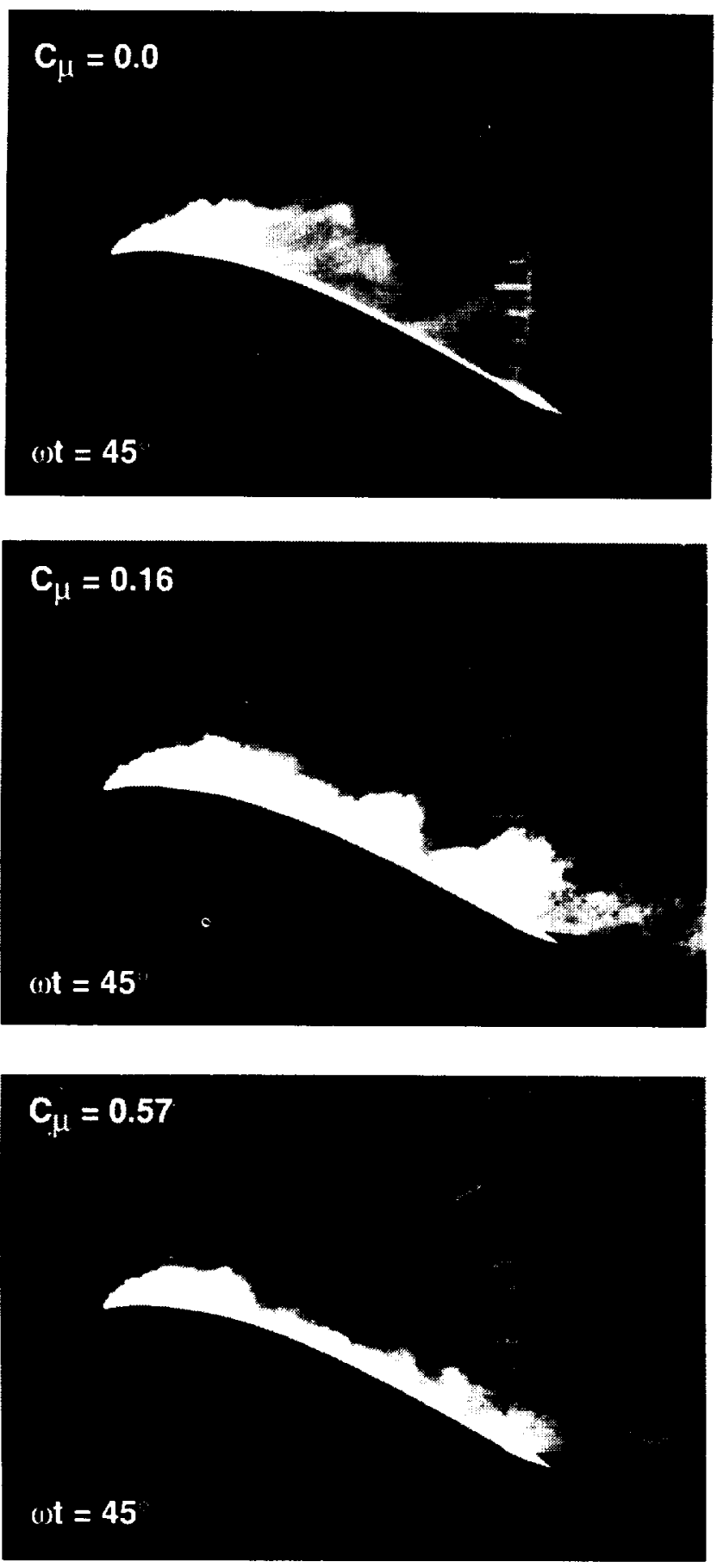

(a) Boundary layer and wake visualization.

Figure 16. Flow visualization and load measurements for $\alpha_{m}=10^{\circ}$ and $k=0.05$ at $(\theta)=45^{\circ}\left(\alpha=17^{\circ}\right)$ with different blowing rates. 

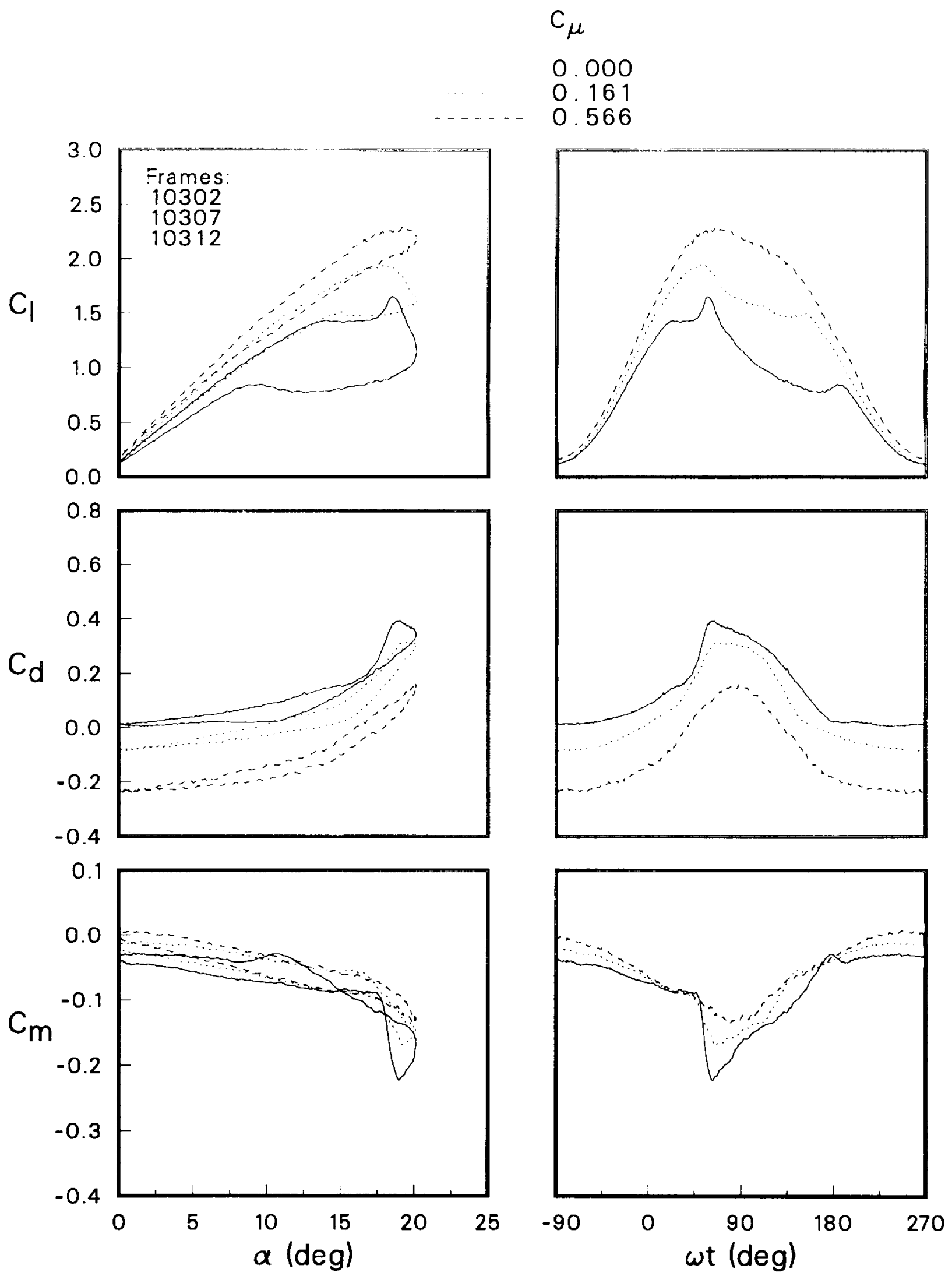

(b) Unsteady force and moment loads.

Figure 16. Concluded. 

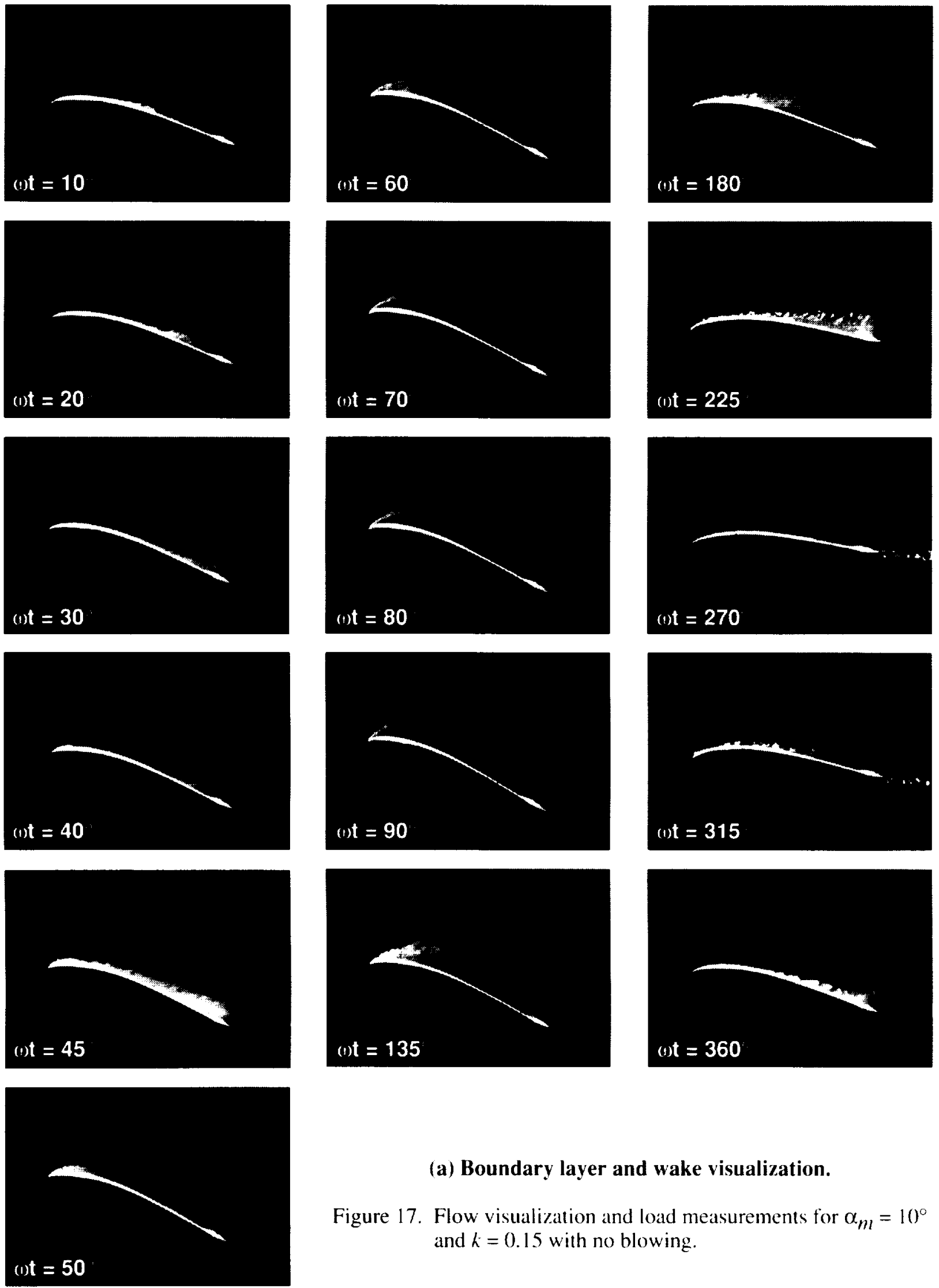

(a) Boundary layer and wake visualization.

Figure 17. Flow visualization and load measurements for $\alpha_{m}=10^{\circ}$ and $k=0.15$ with no blowing. 


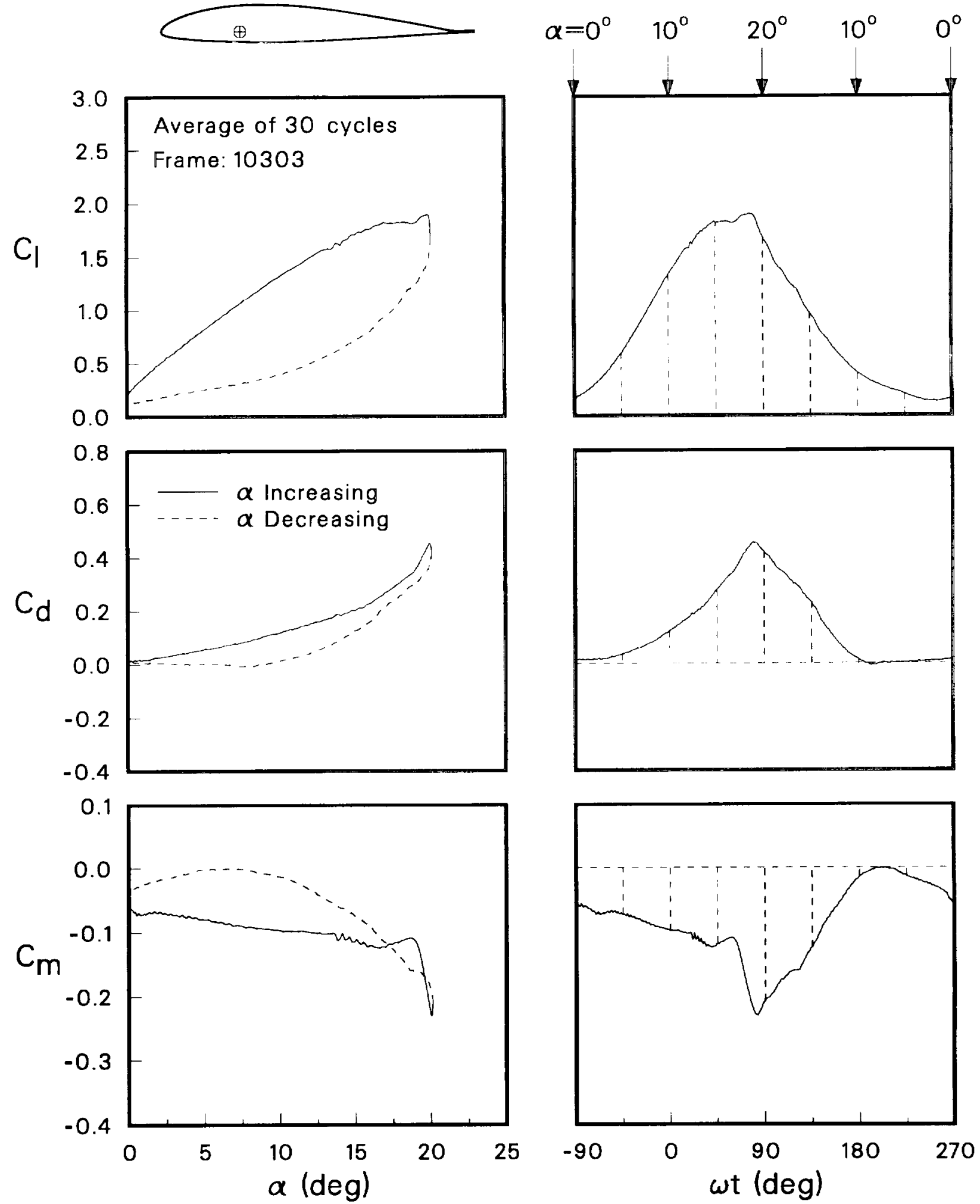

(b) Unsteady force and moment loads.

Figure 17. Concluded. 

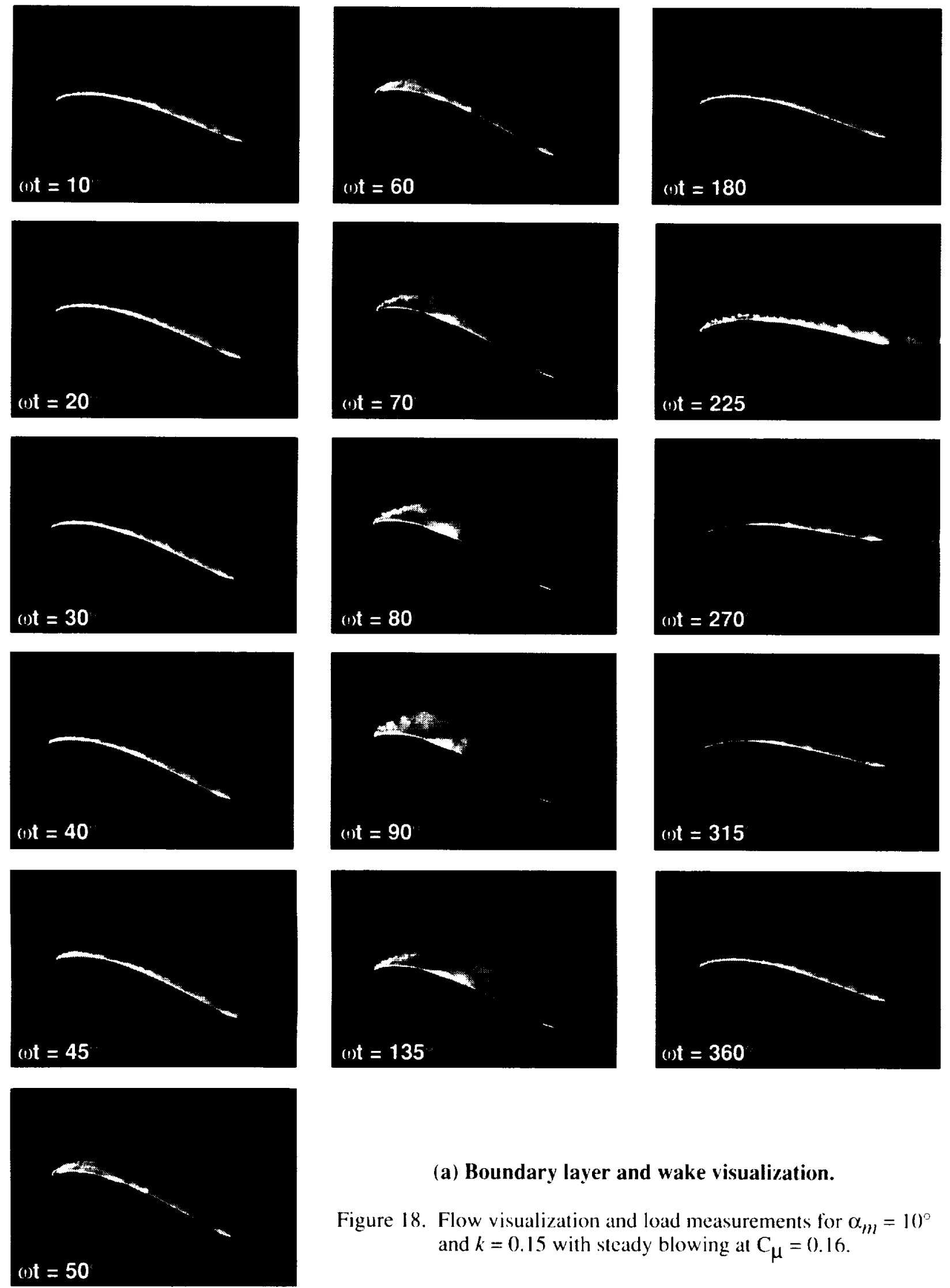

(a) Boundary layer and wake visualization.

Figure 18. Flow visualization and load measurements for $\alpha_{m}=10^{\circ}$ and $k=0.15$ with steady blowing at $\mathrm{C}_{\mu}=0.16$. 


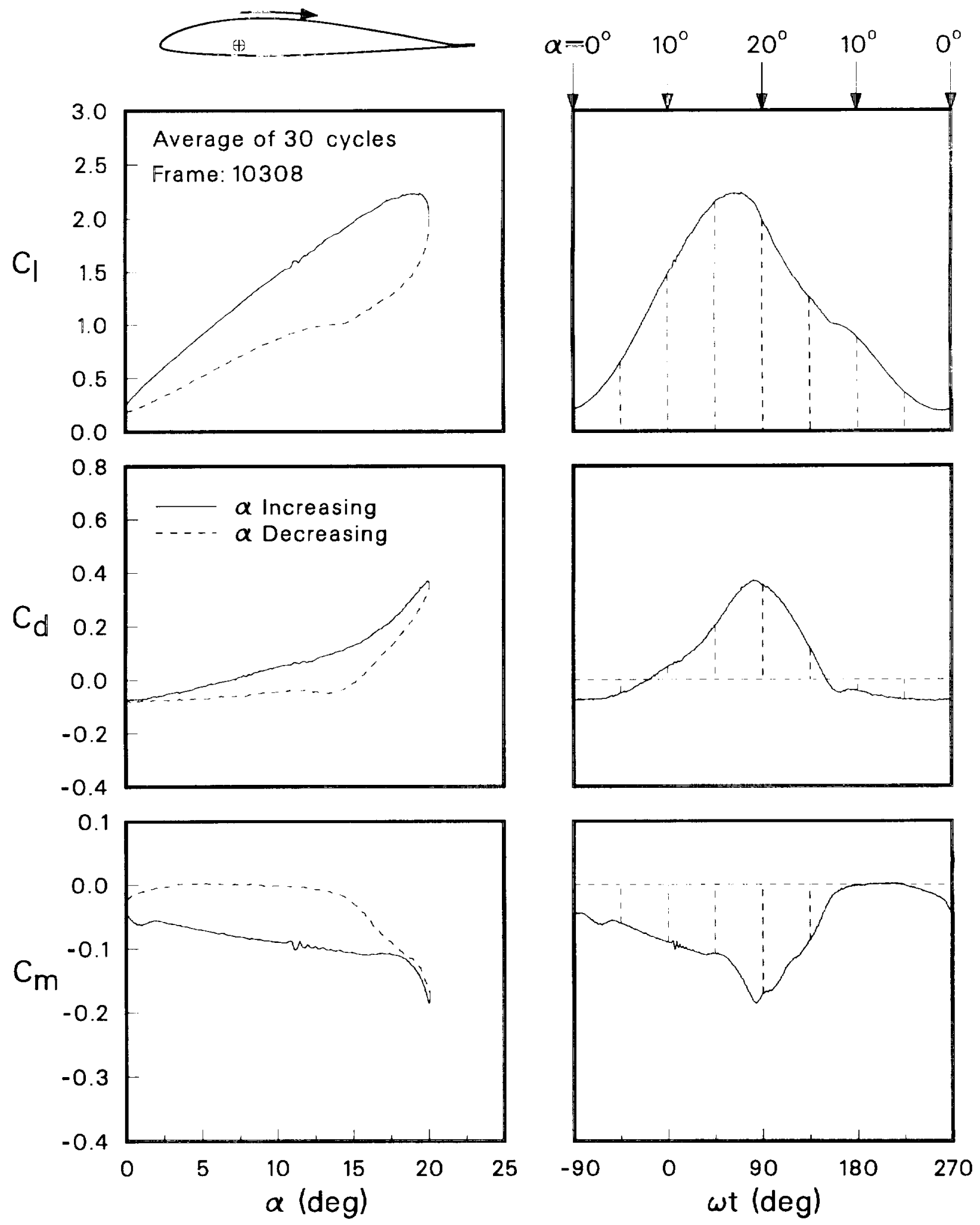

(b) Unsteady force and moment loads.

Figure 18. Concluded. 

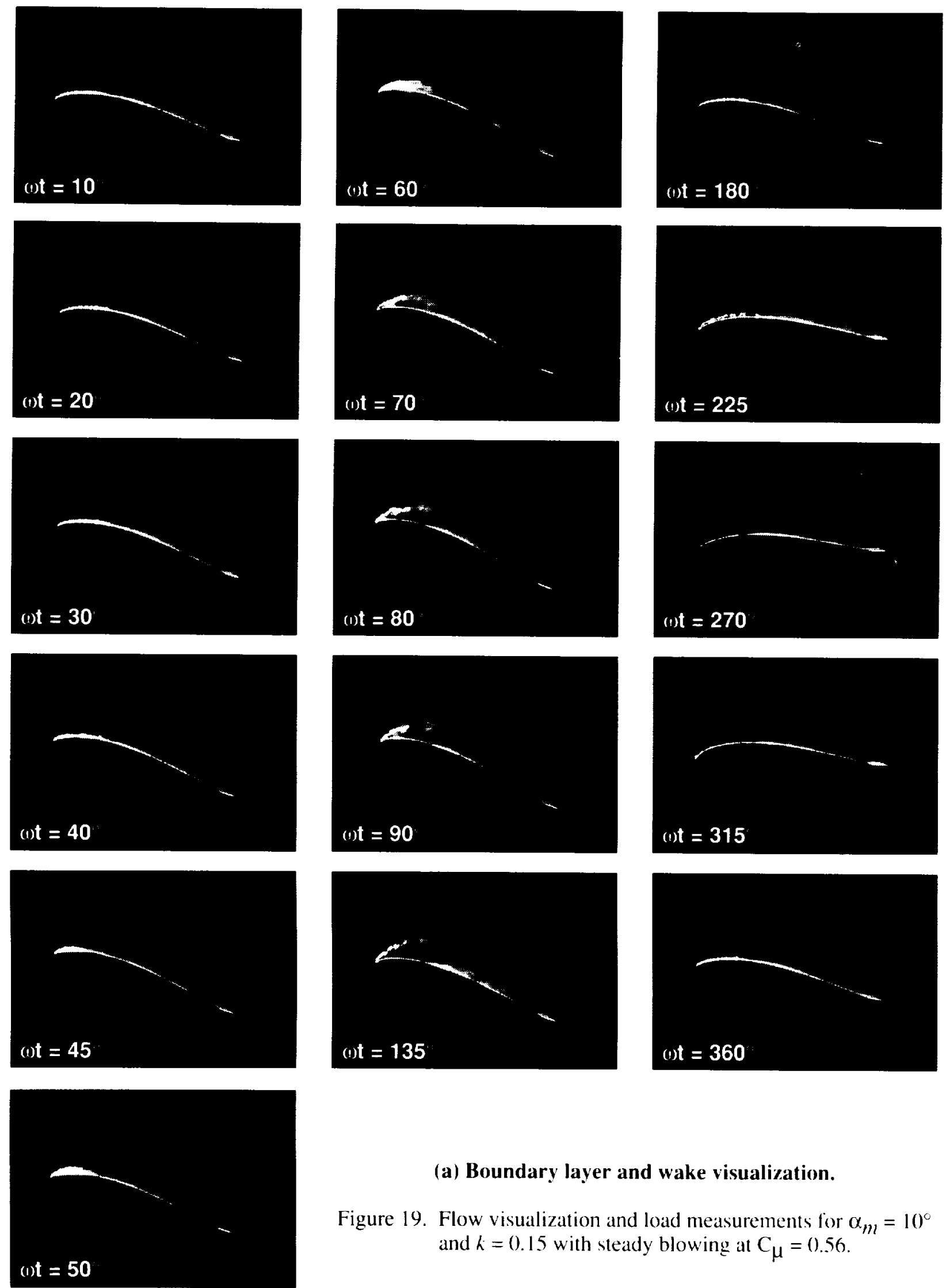

(a) Boundary layer and wake visualization.

Figure 19. Flow visualization and load measurements for $\alpha_{m}=10^{\circ}$ and $k=0.15$ with steady blowing at $C_{\mu}=0.56$. 


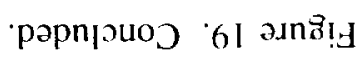

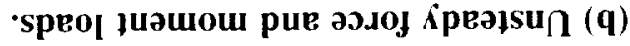

(6әp) Im

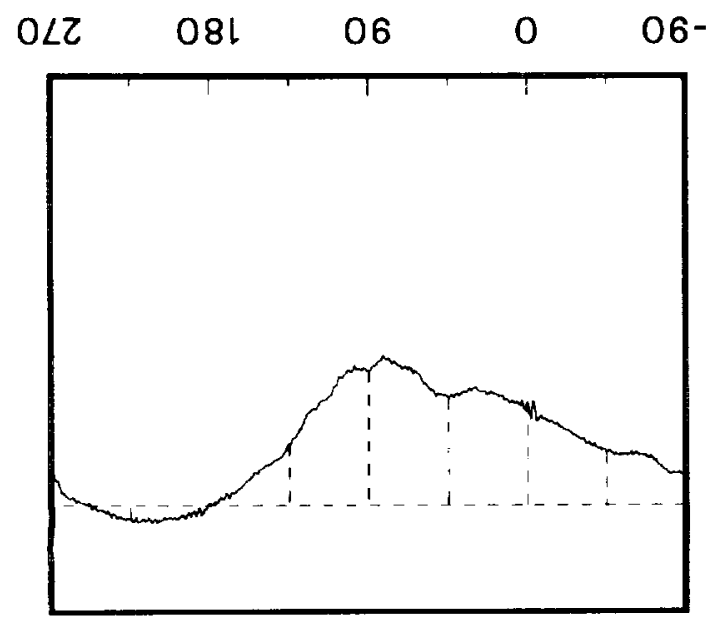

(Бәр) 0

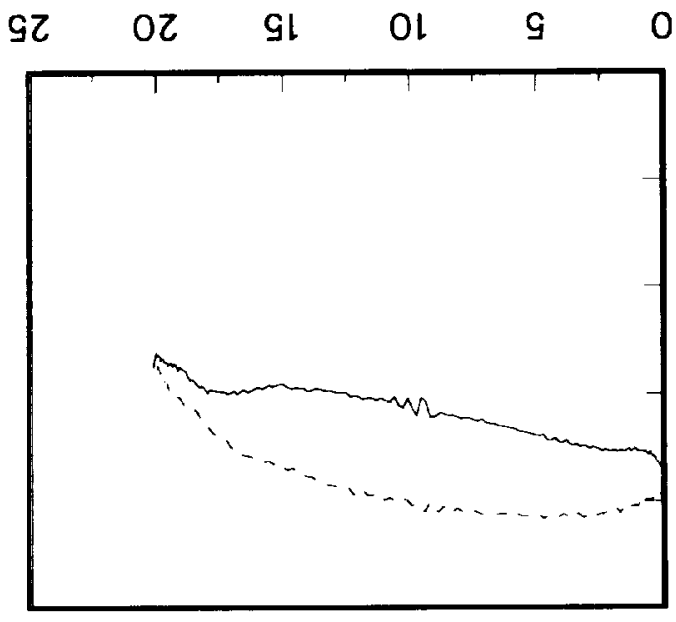

$80-$
80
20
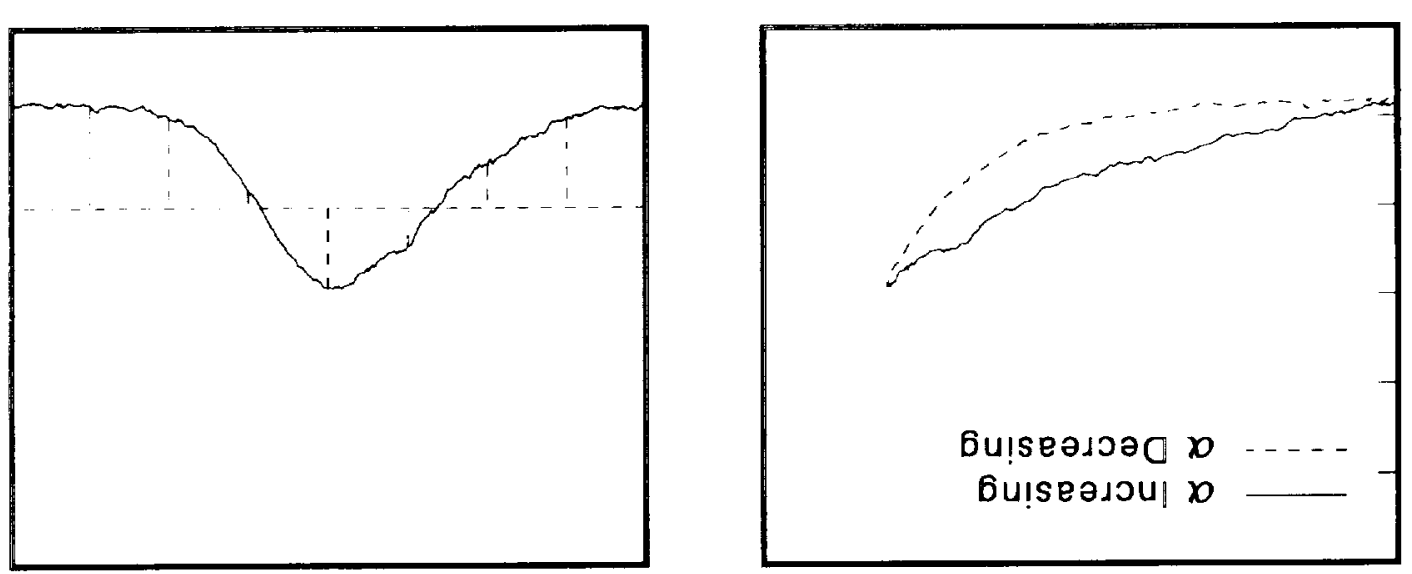

$1 \cdot 0$

b० -

Z.0-

0.0

$2 \cdot 0$

Po

to

90
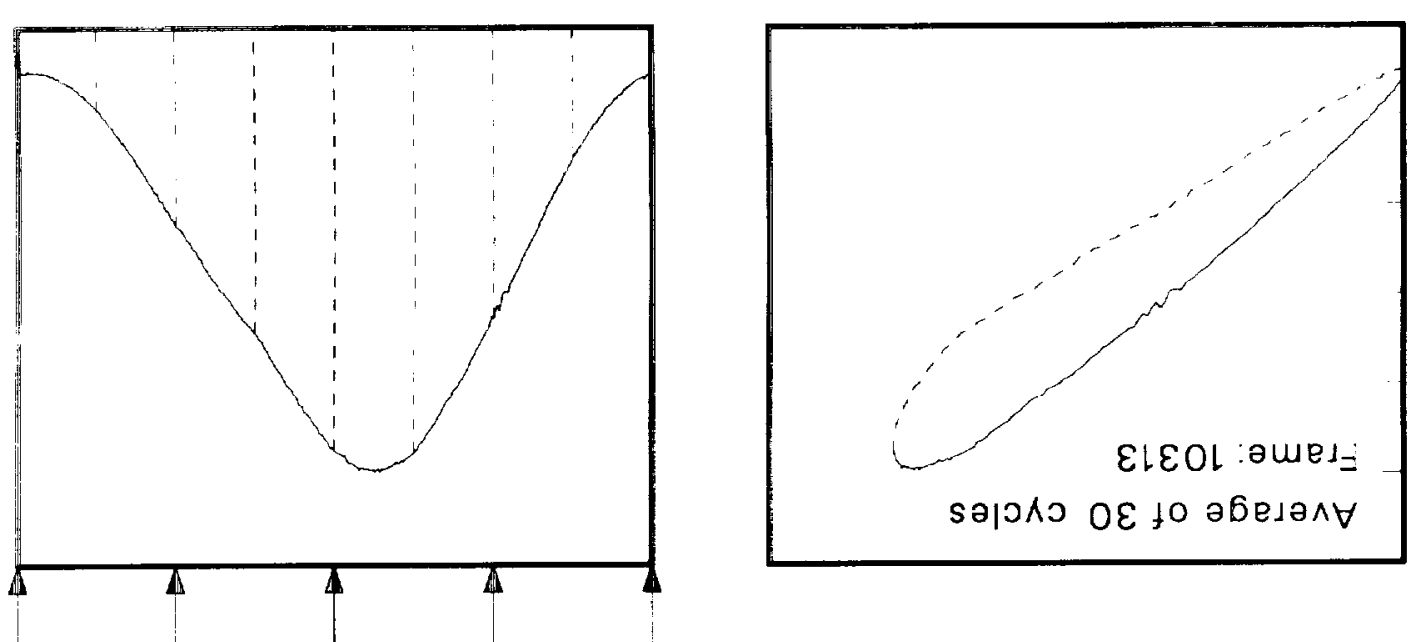

80

0.0

g.0

$0 \%$

si lo

02

G'2

${ }_{0} \mathrm{O} \quad{ }_{0} \mathrm{Ol} \quad{ }_{0} \mathrm{OZ} \quad{ }_{\mathrm{O}} \mathrm{Ol} \quad{ }_{0} \mathrm{O}=0$

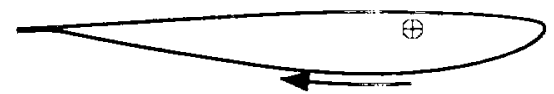



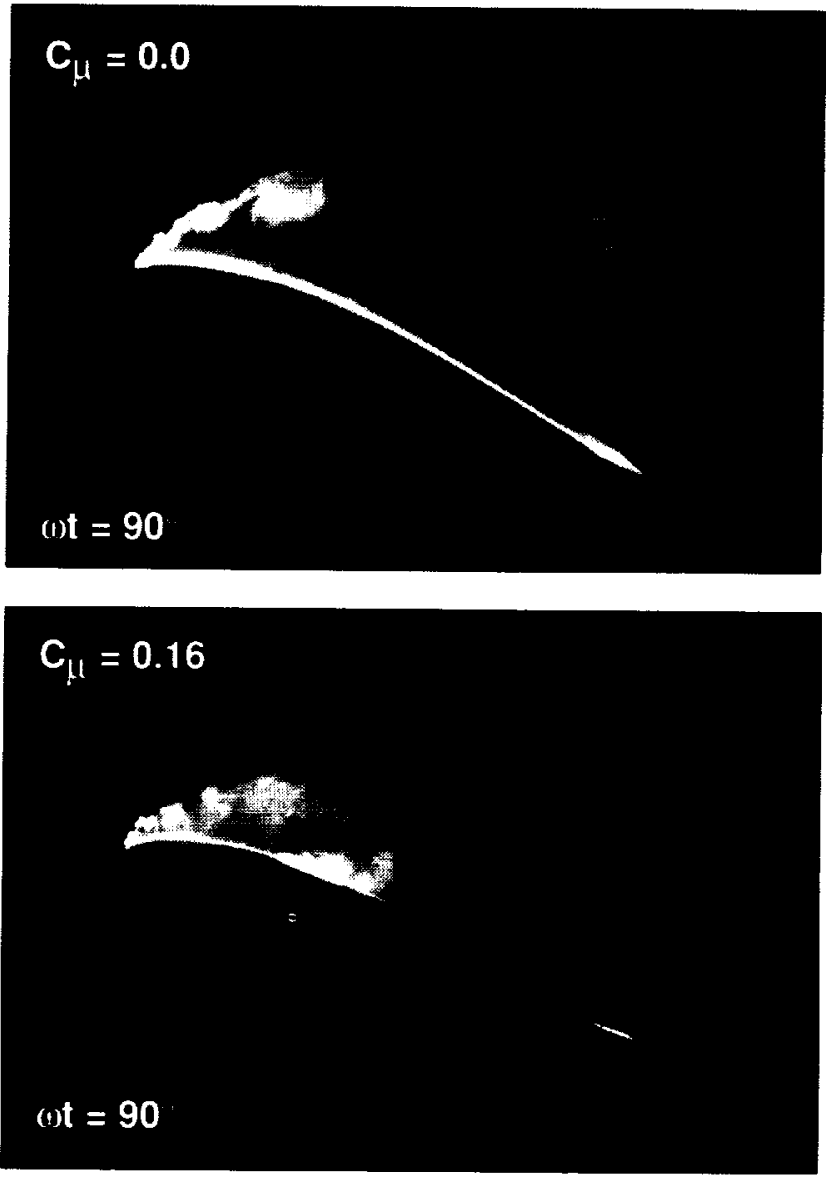

$$
c_{\mu}=0.56
$$

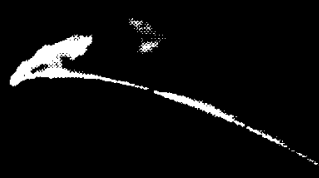

(1)t $=90$

(a) Boundary layer and wake visualization.

Figure 20. Flow visualization and load measurements for $\alpha_{m}=10^{\circ}$ and $k=0.15$ at $\omega t=90^{\circ}\left(\alpha=20^{\circ}\right)$ with different blowing rates. 


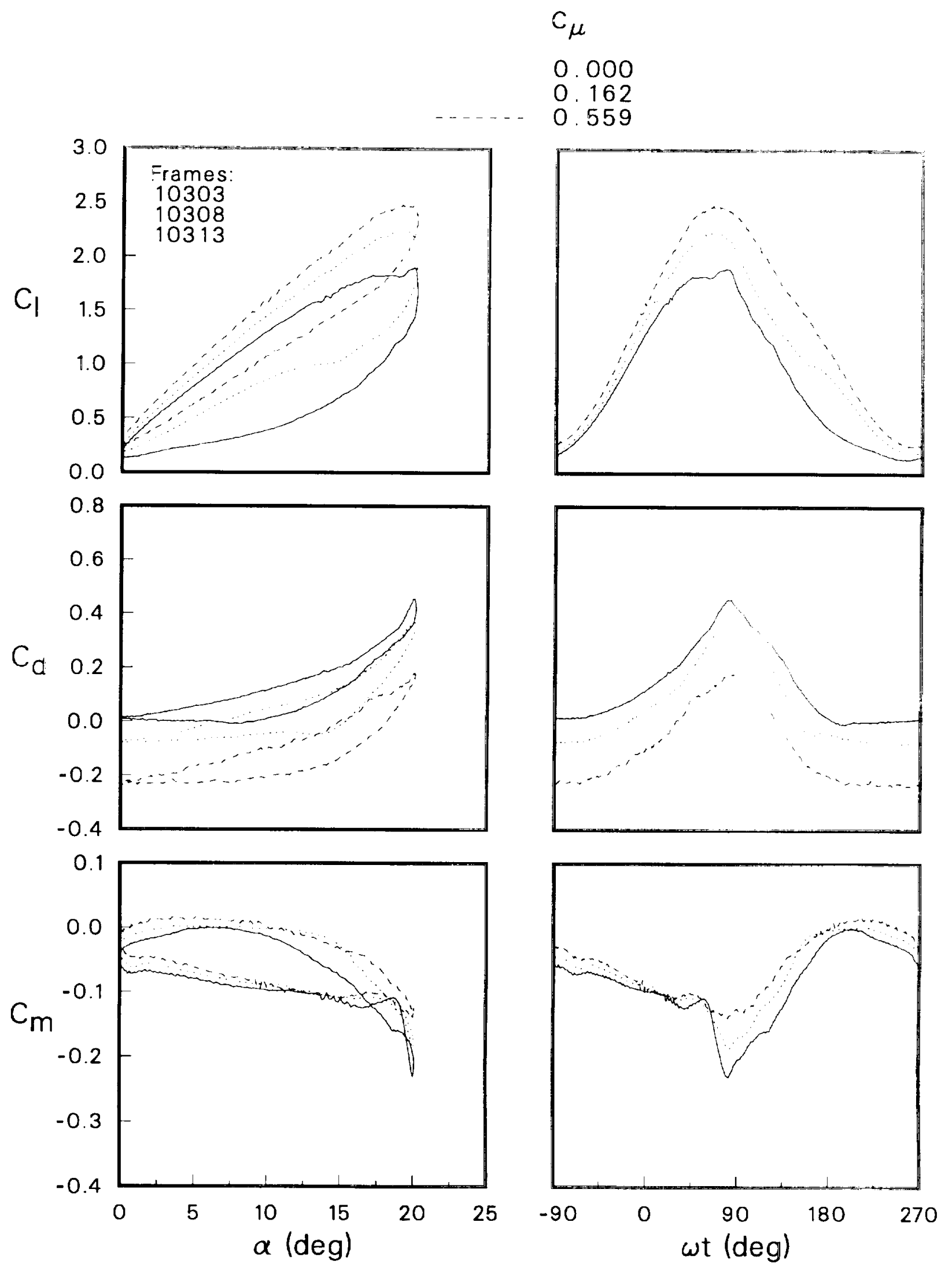

(b) Unsteady force and moment loads.

Figure 20. Concluded. 

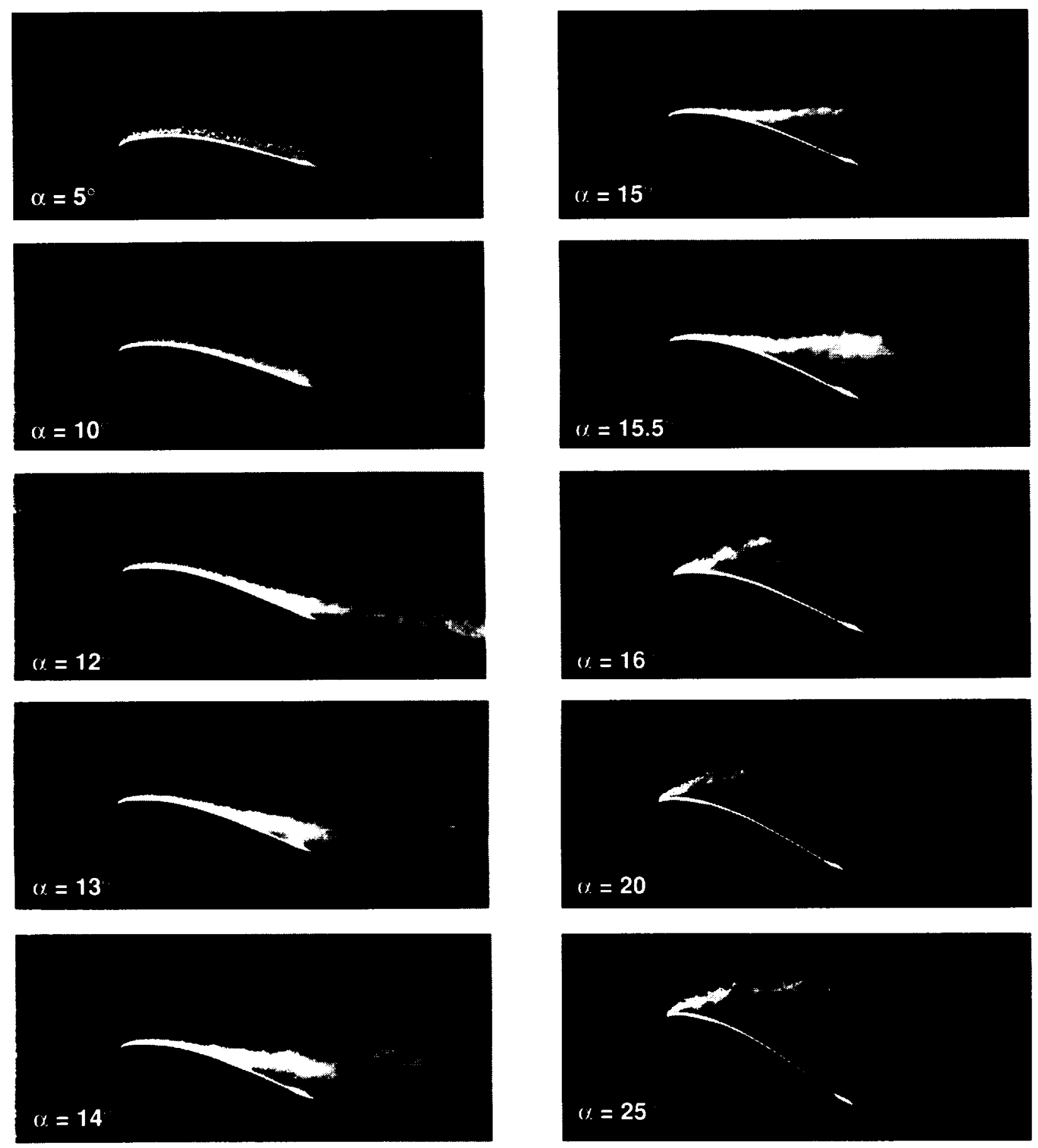

(a) Boundary layer and wake visualization.

Figure 21. Flow visualization and load measurements for $\alpha_{m}=15^{\circ}$ and $k=0.007$ with no blowing. 


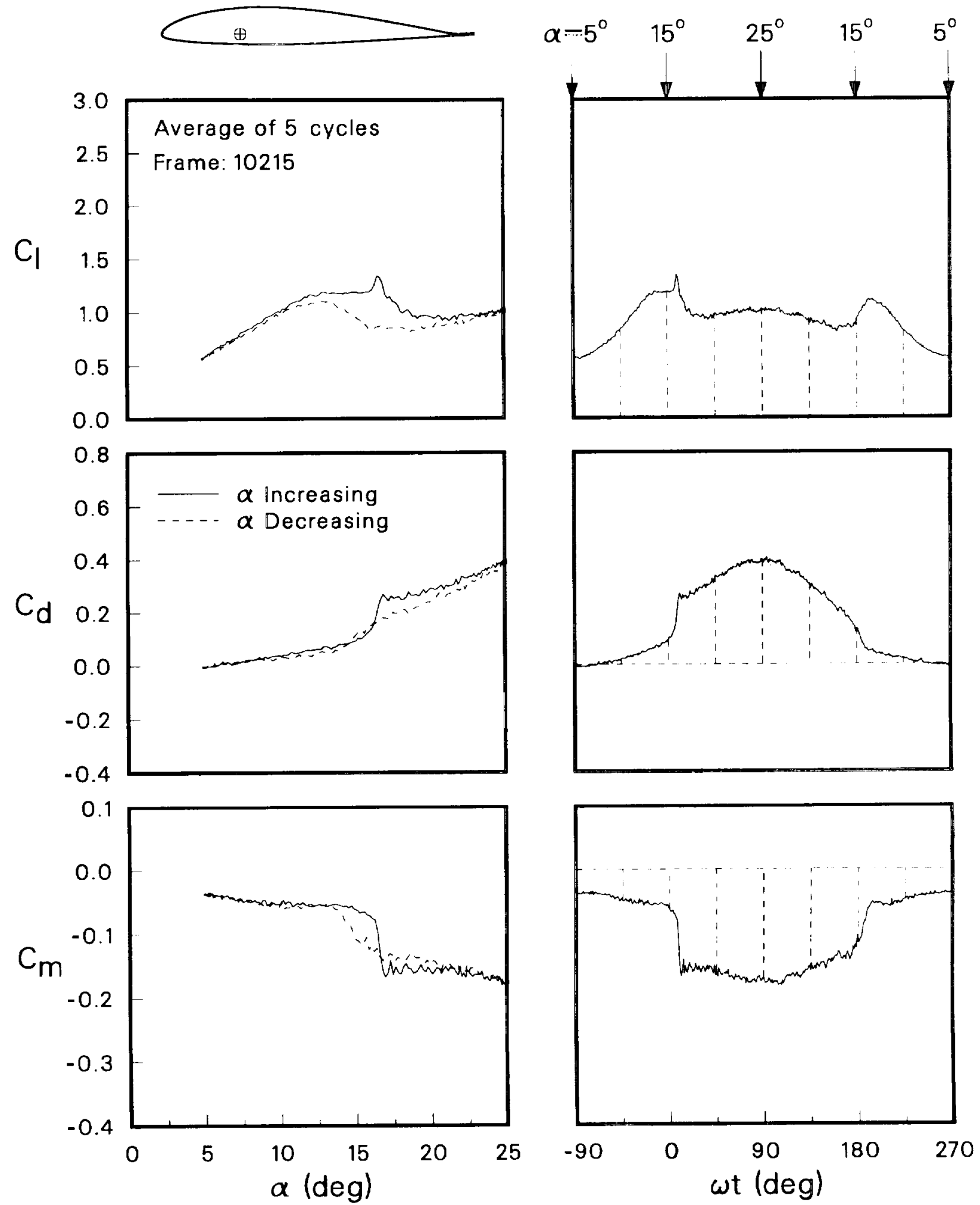

(b) Unsteady force and moment loads.

Figure 21. Concluded. 

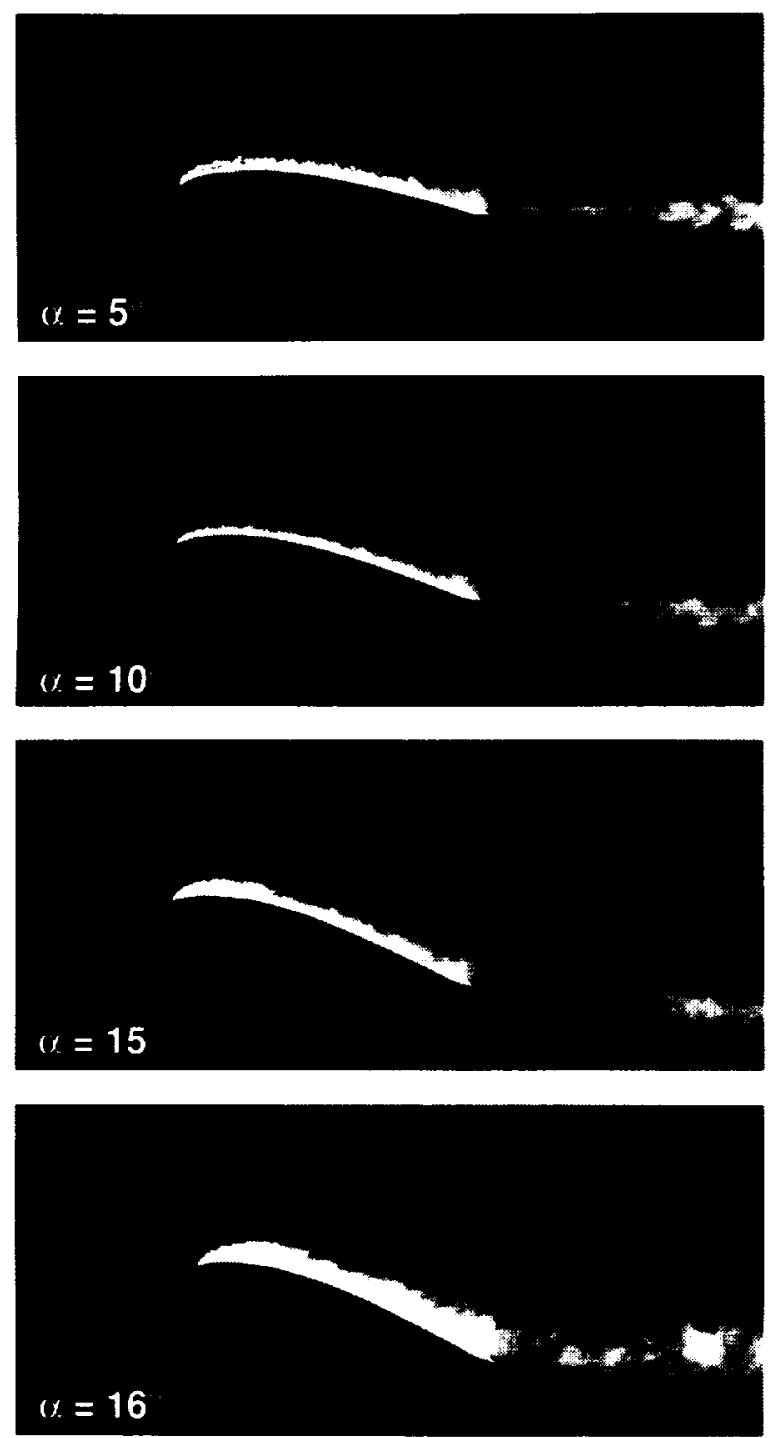
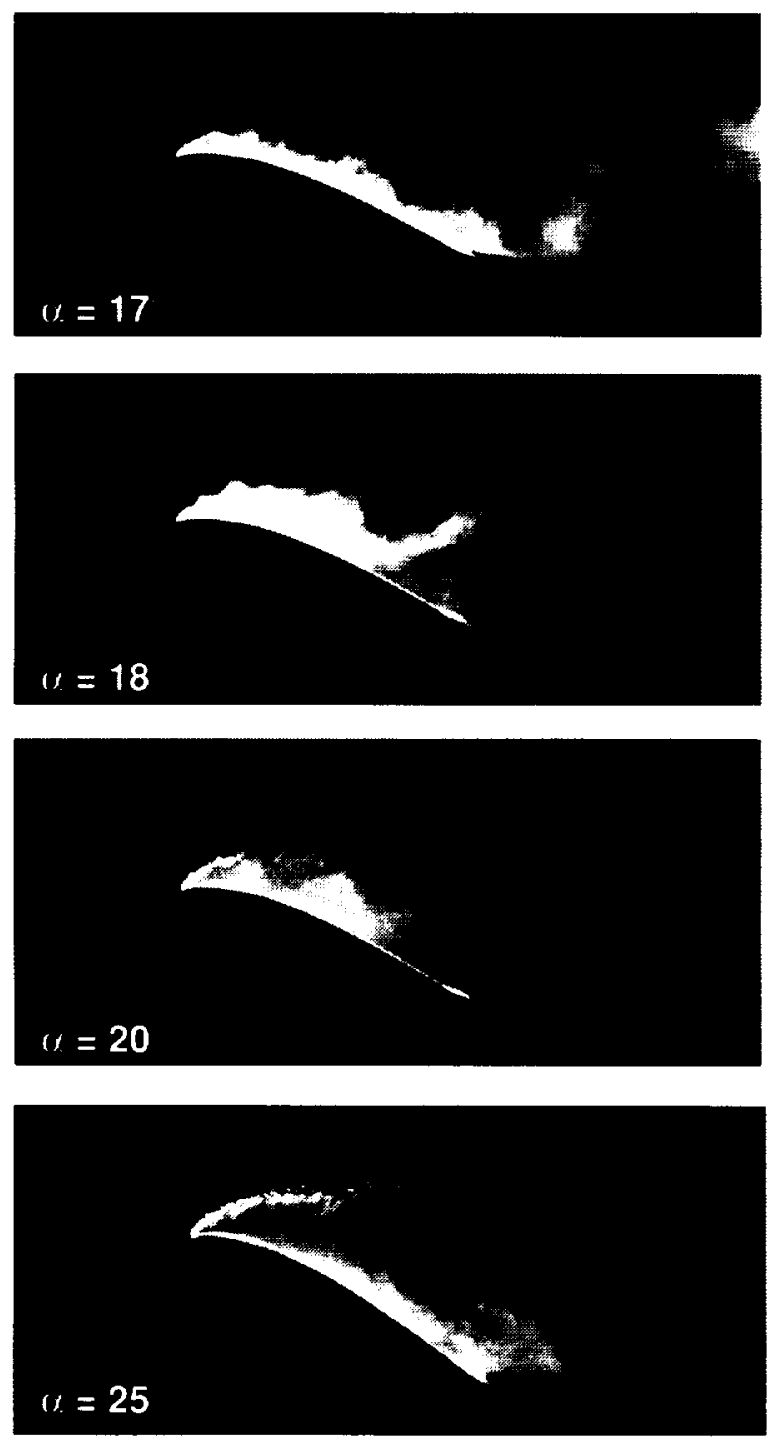

(a) Boundary layer and wake visualization.

Figure 22. Flow visualization and load measurements for $\alpha_{m}=15^{\circ}$ and $k=0.007$ with steady blowing at $C_{\mu}=0.19$. 


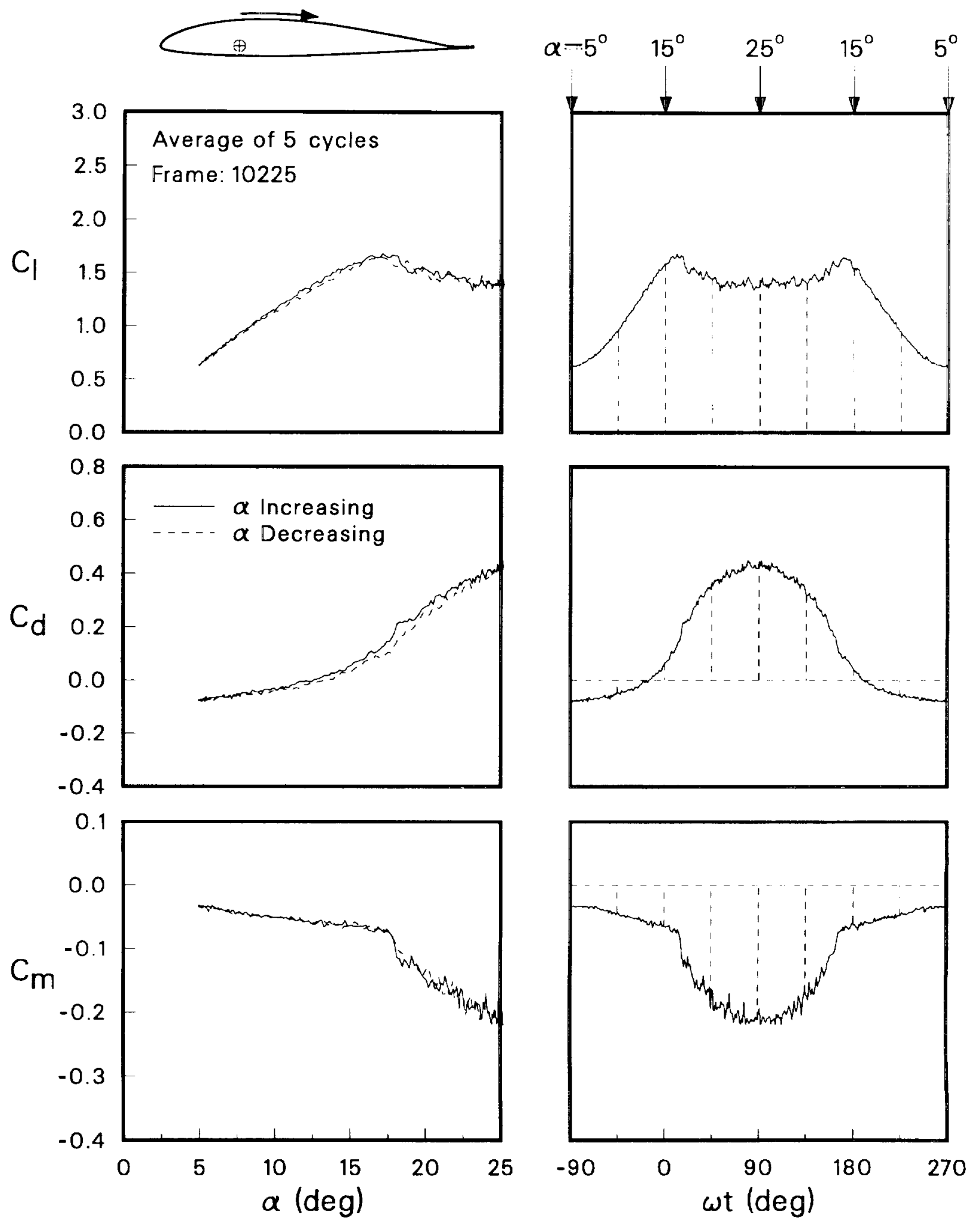

(b) Unsteady force and moment loads.

Figure 22. Concluded. 

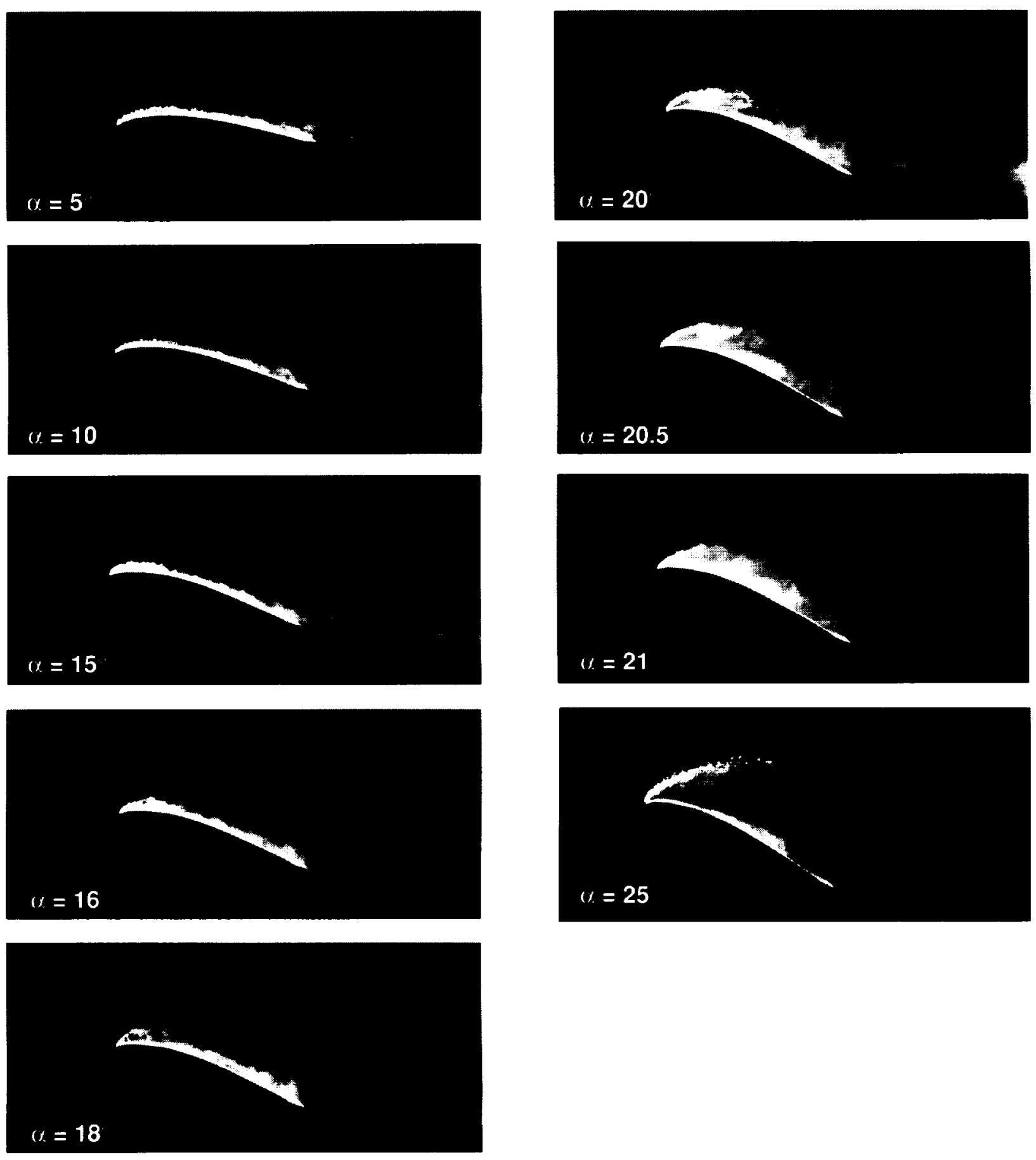

(a) Boundary layer and wake visualization.

Figure 23. Flow visualization and load measurements for $\alpha_{m}=15^{\circ}$ and $k=0.006$ with steady blowing at $C_{\mu}=0.64$. 

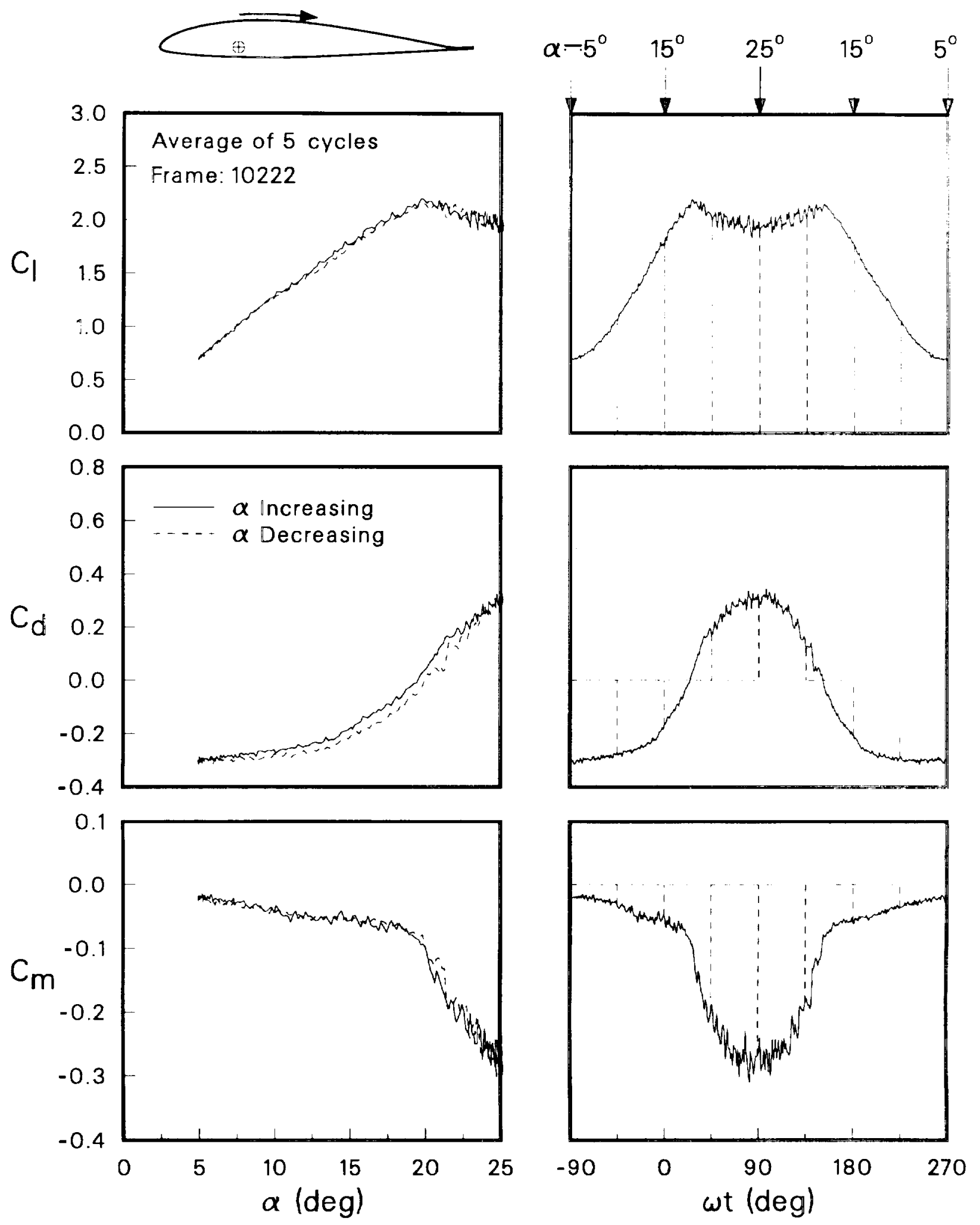

(b) Unsteady force and moment loads.

Figure 23. Concluded. 

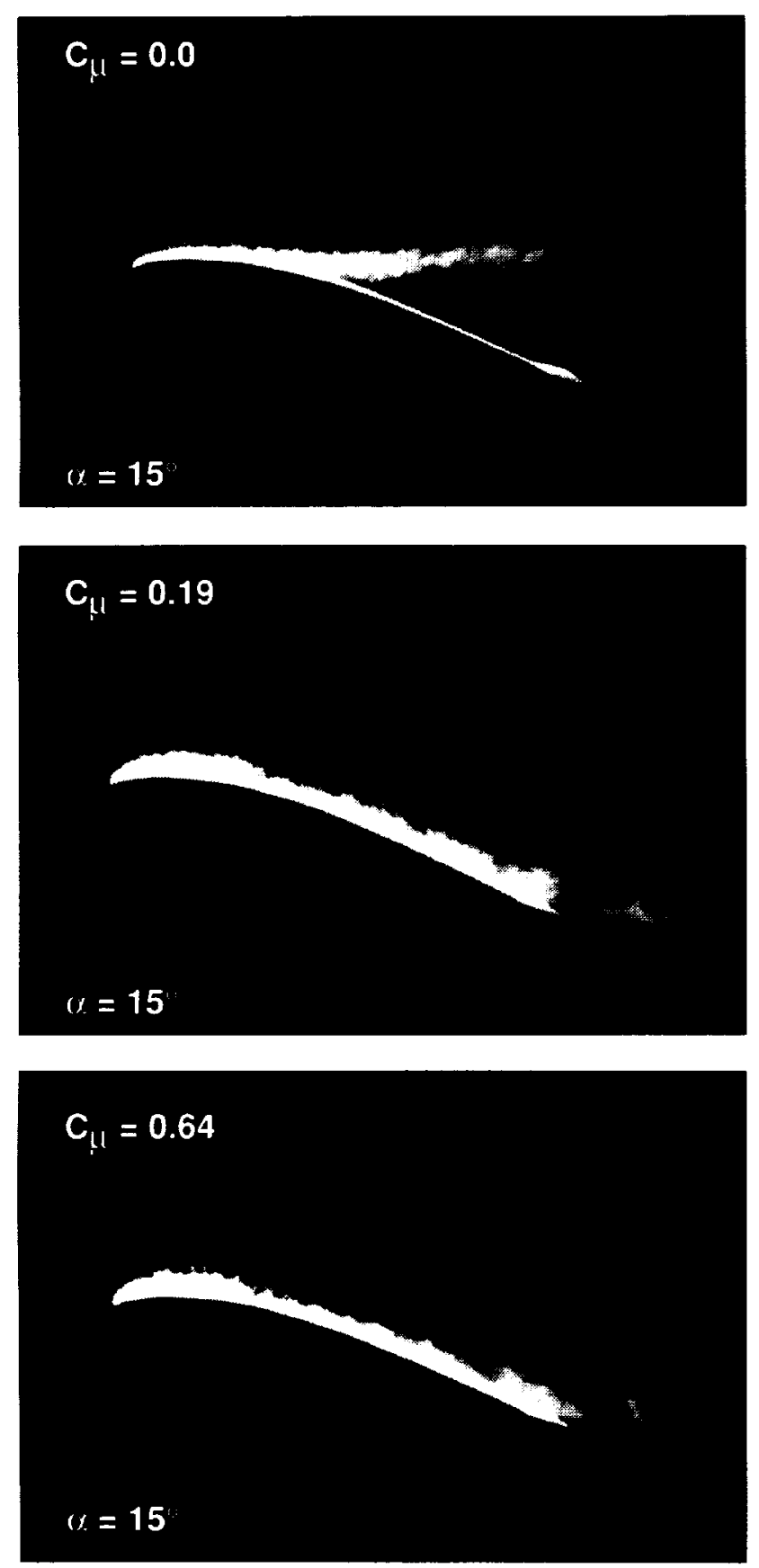

(a) Boundary layer and wake visualization.

Figure 24. Flow visualization and load measurements for $\alpha_{m}=15^{\circ}$ and $k=0.007$ (nominal) at $\alpha=15^{\circ}$ with different blowing raltes. 
spro| luauou pur a.d(I iprajsu (q)

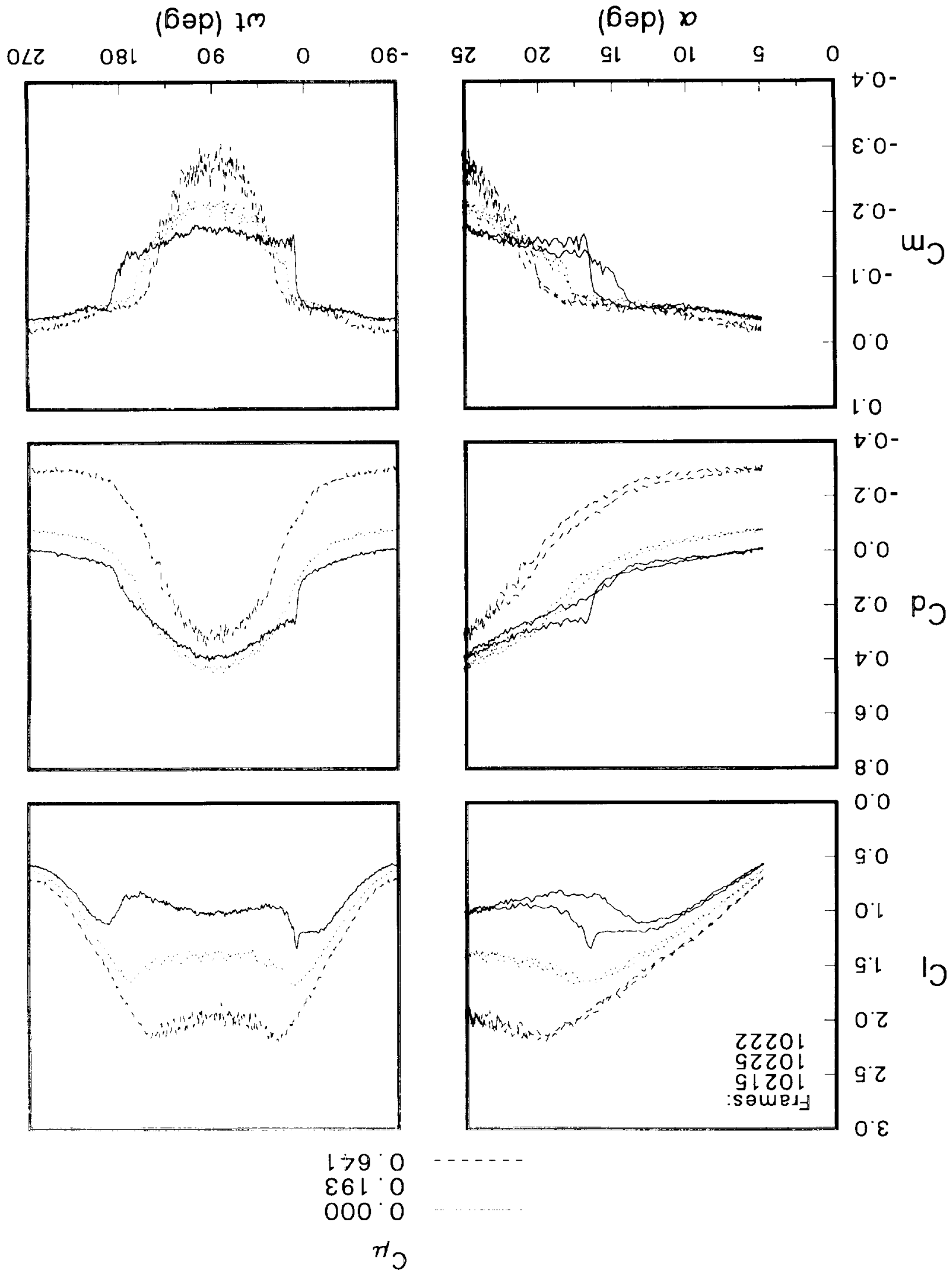



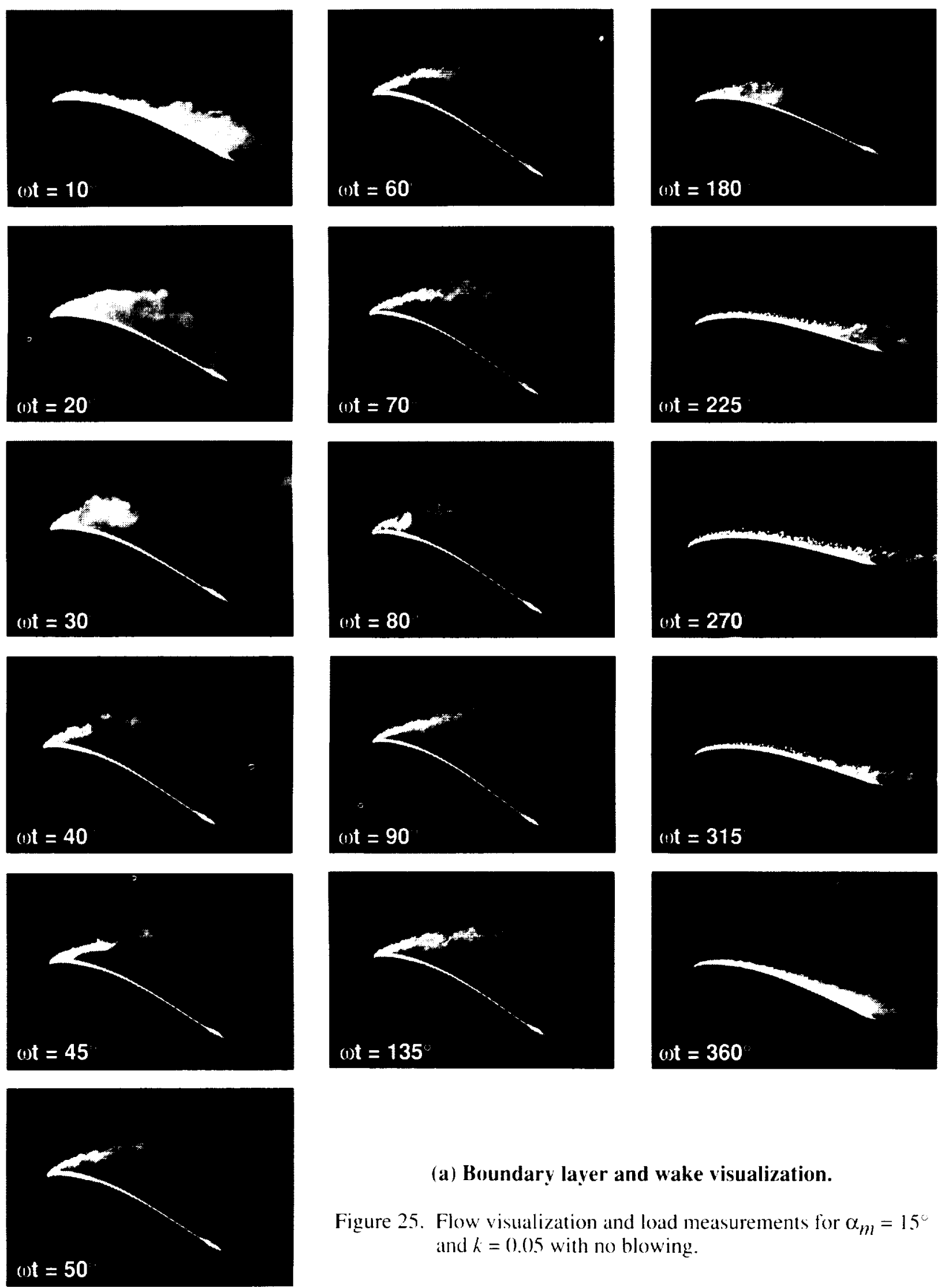

(a) Boundary layer and wake visualization.

Figure 25. Flow visualization and load measurements for $\alpha_{m}=15^{\circ}$ and $k=0.05$ with no blowing. 


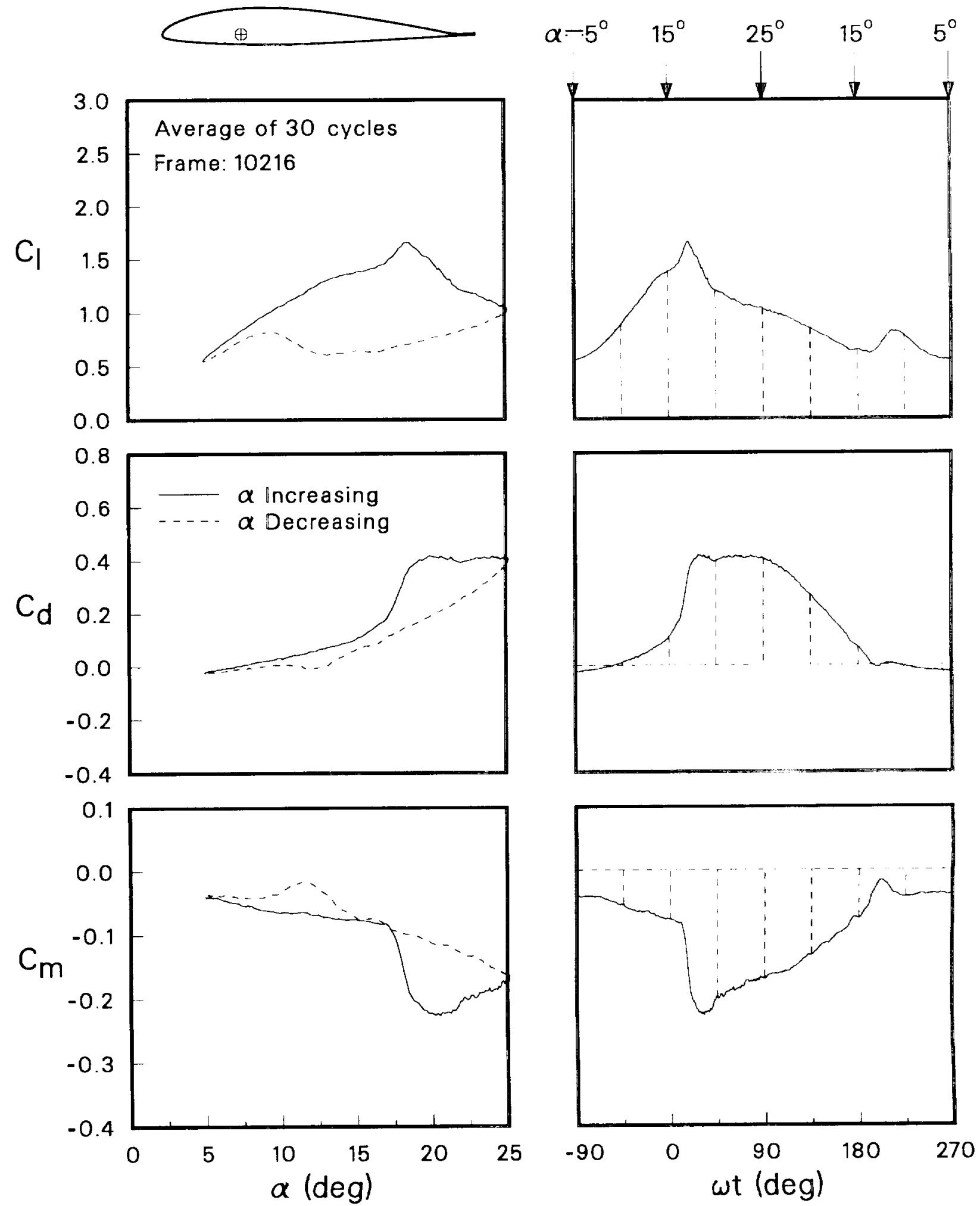

(b) Unsteady force and moment loads.

Figure 25. Concluded. 

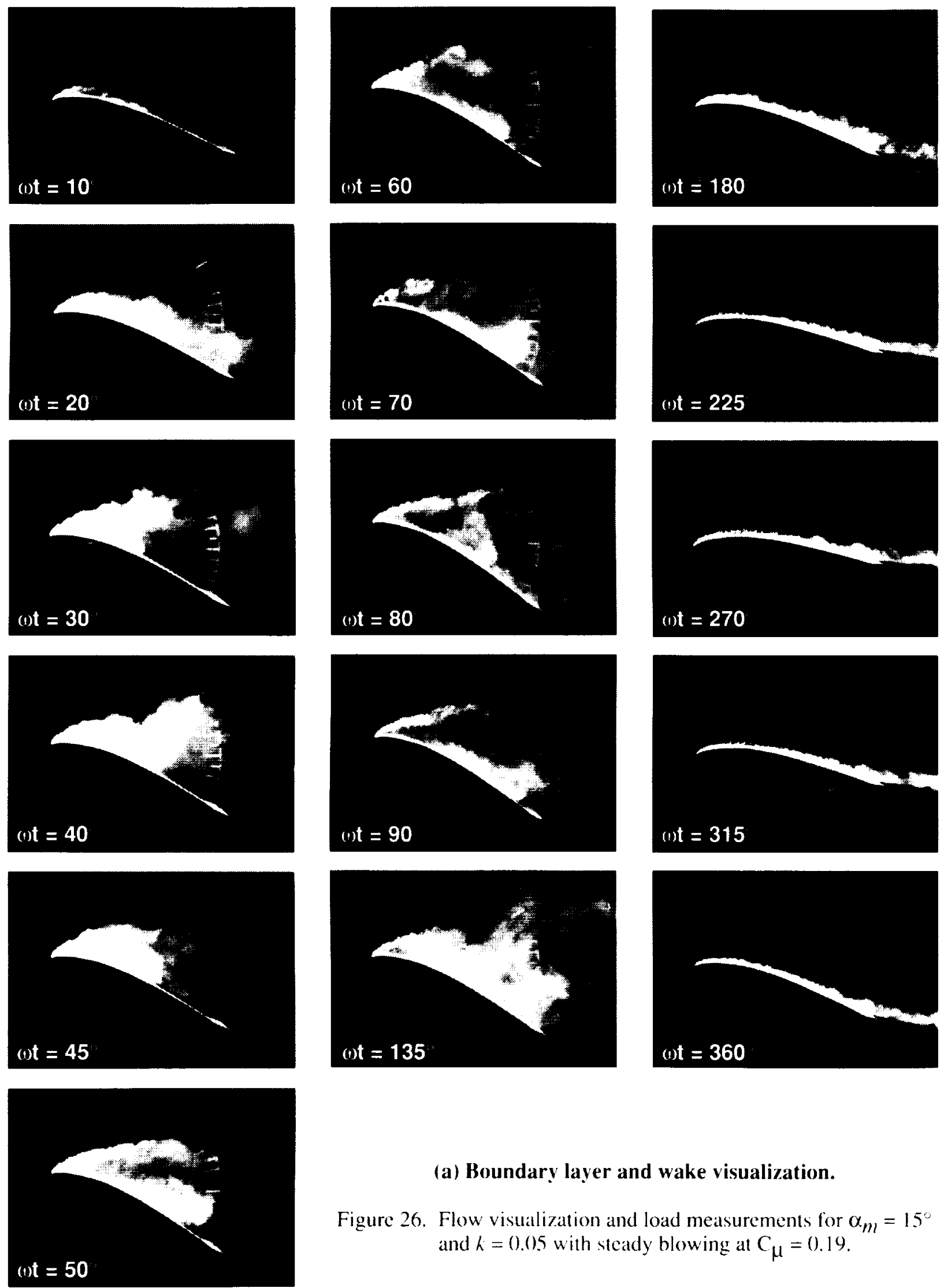

(a) Boundary layer and wake visualization.

Figure 26. Flow visualization and load measurements for $\alpha_{m}=15^{\circ}$ and $k=0.0 .5$ with steady blowing at $C_{\mu}=0.19$. 

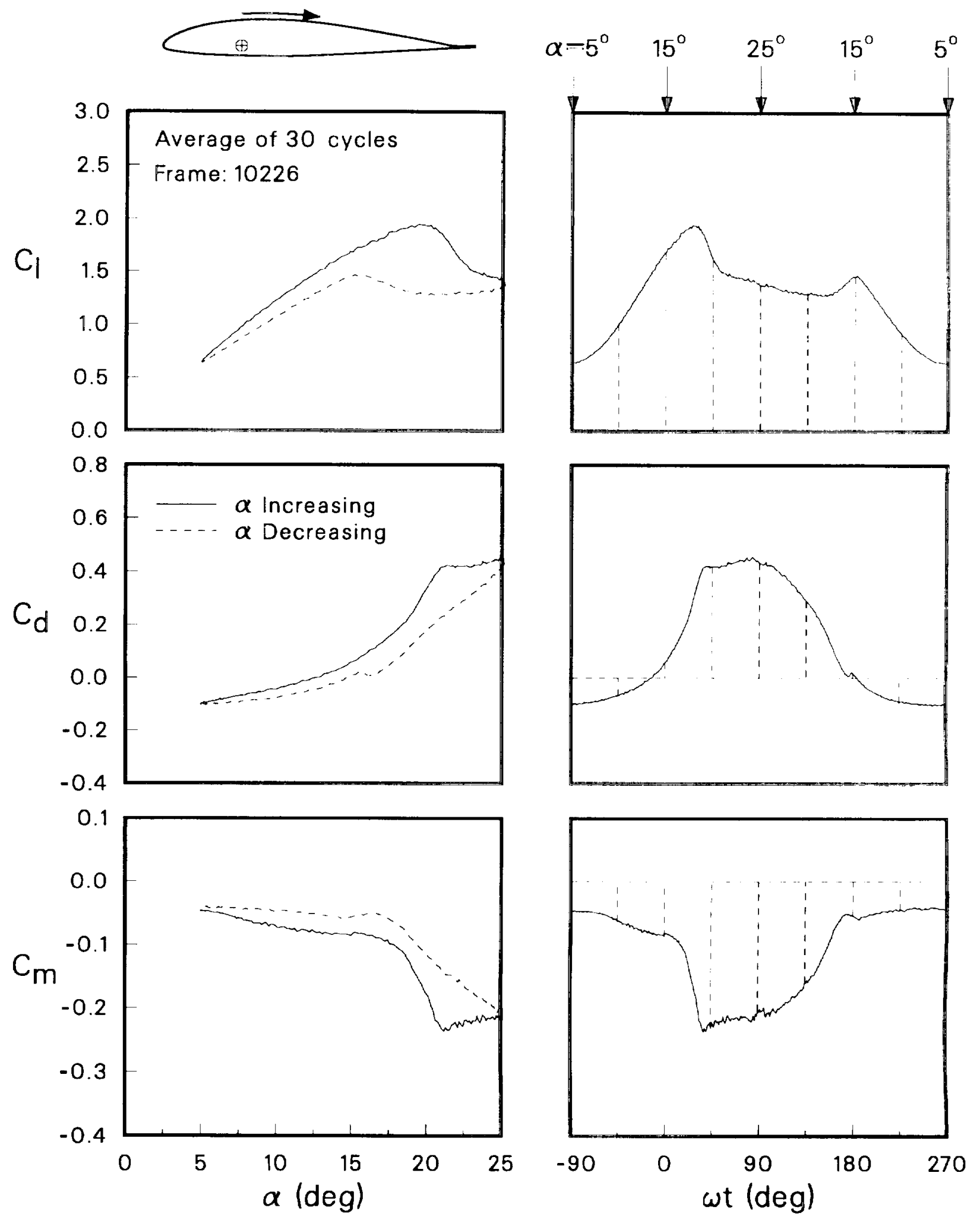

(b) Unsteady force and moment loads.

Figure 26. Concluded. 

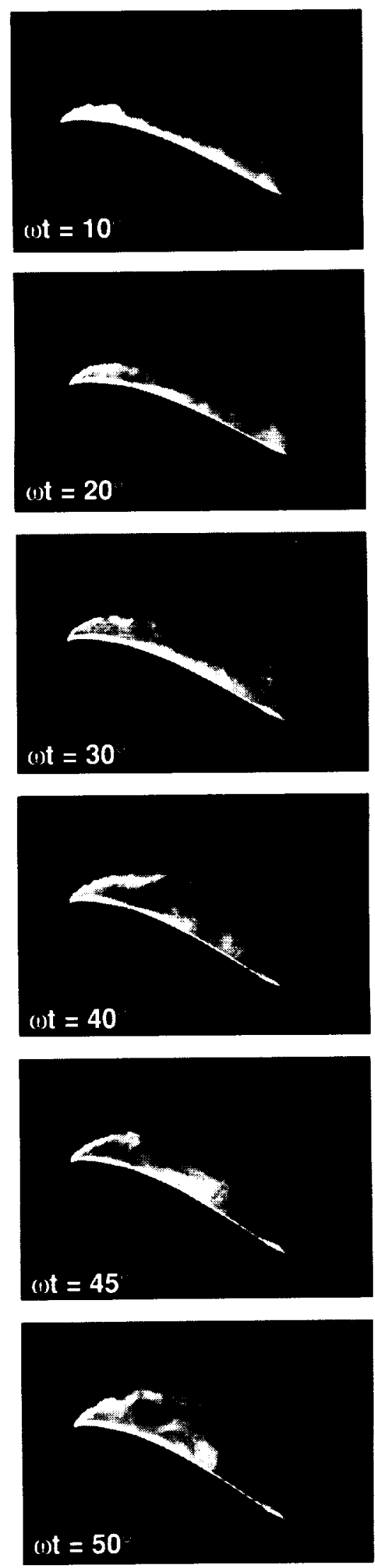
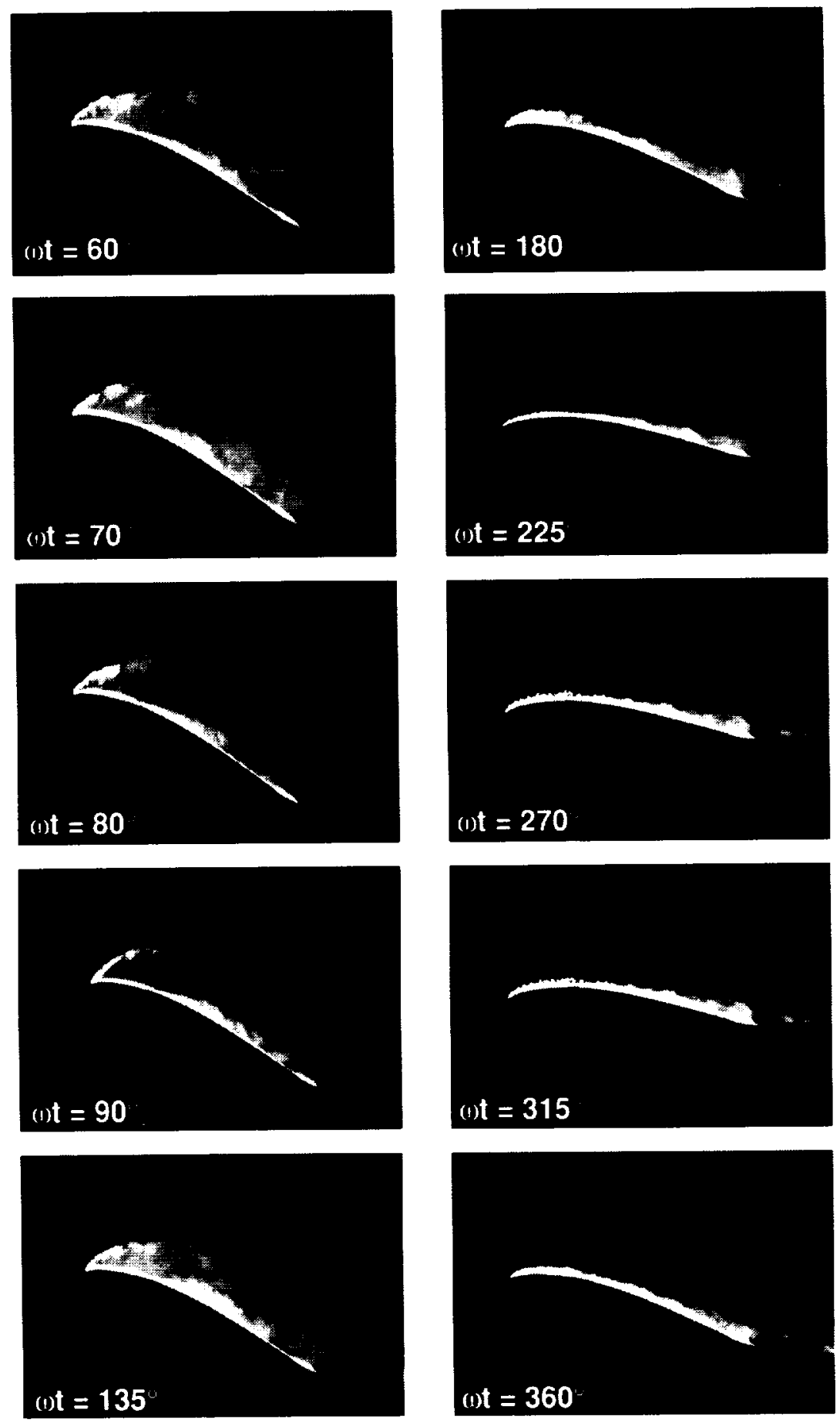

(a) Boundary layer and wake visualization.

Figure 27. Flow visualization and load measurements for $\alpha_{m}=15^{\circ}$ and $k=0.05$ with steady blowing at $\mathrm{C}_{\mu}=0.66$. 

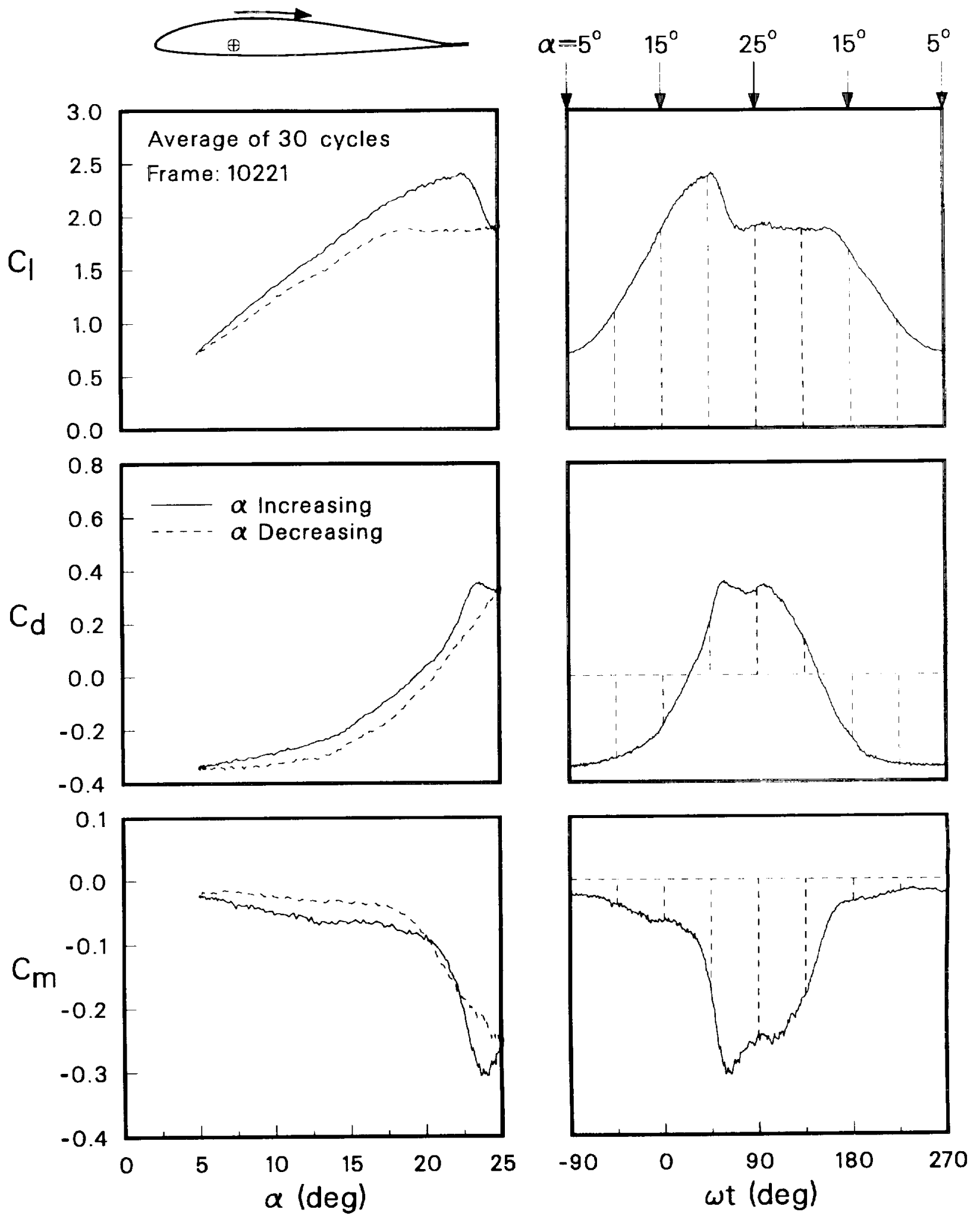

(b) Unsteady force and moment loads.

Figure 27. Concluded. 

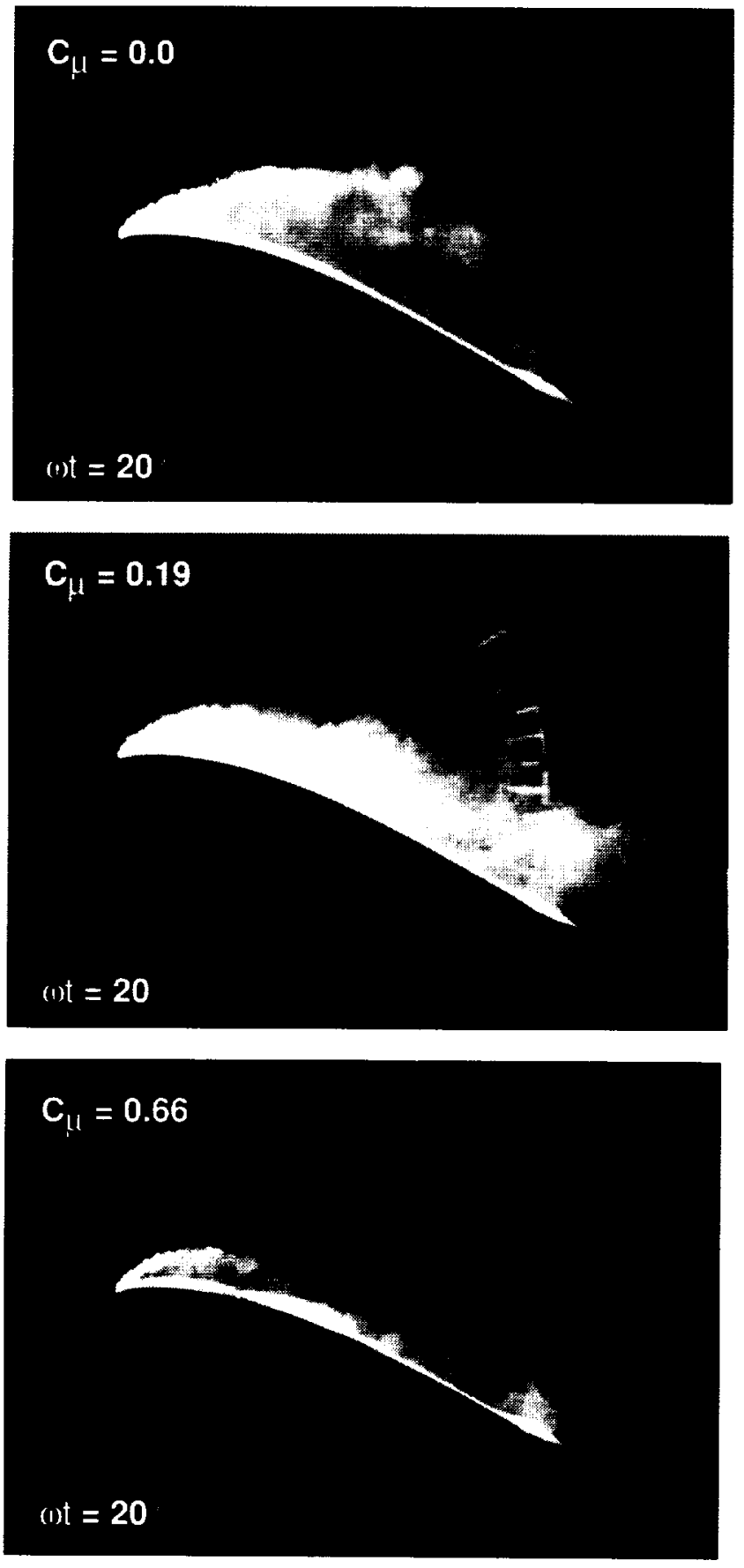

(a) Boundary layer and wake visualization.

Figure 28. Flow visualization and load measurements for $\alpha_{m}=15^{\circ}$ and $k=0.05$ at $\omega t=20^{\circ}\left(\alpha=18.4^{\circ}\right)$ with different blowing rates. 


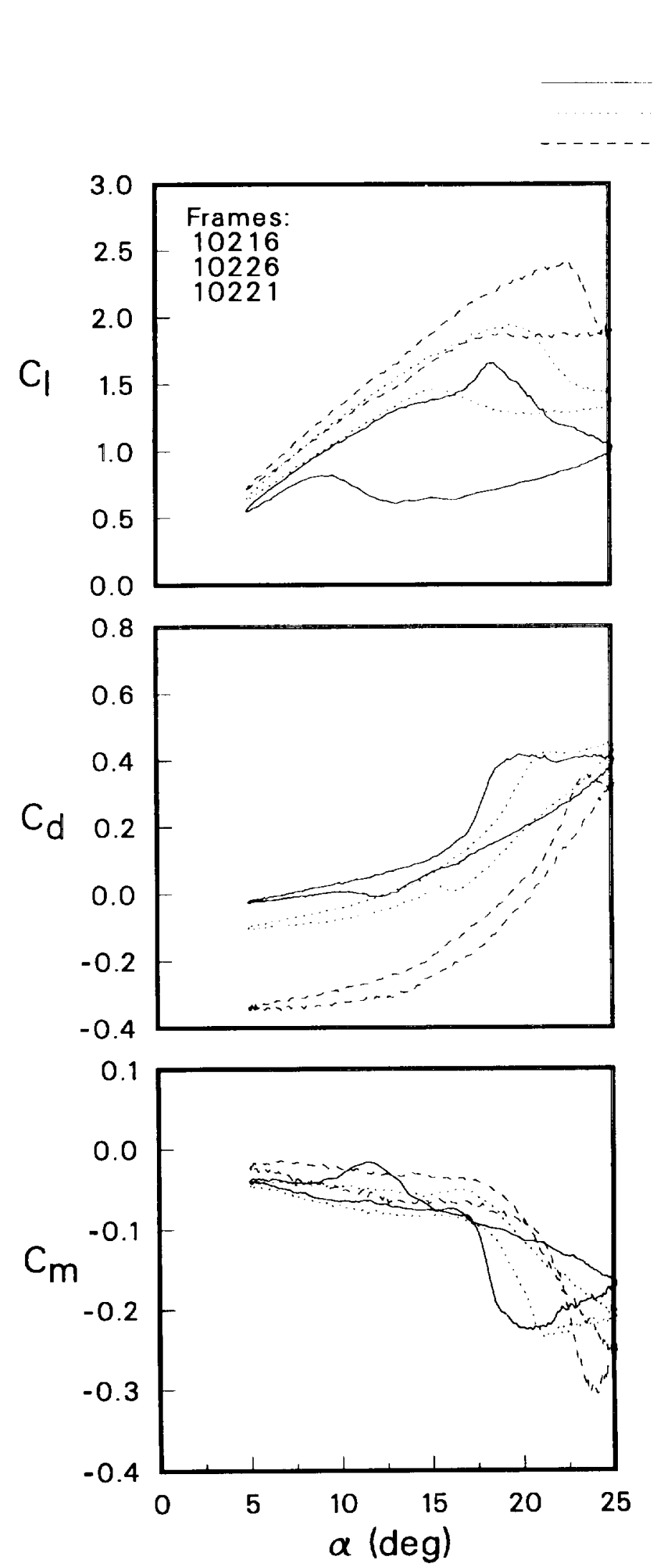

$$
\begin{aligned}
& \mathrm{C}_{\mu} \\
& 0.000 \\
& 0.193 \\
& 0.662
\end{aligned}
$$
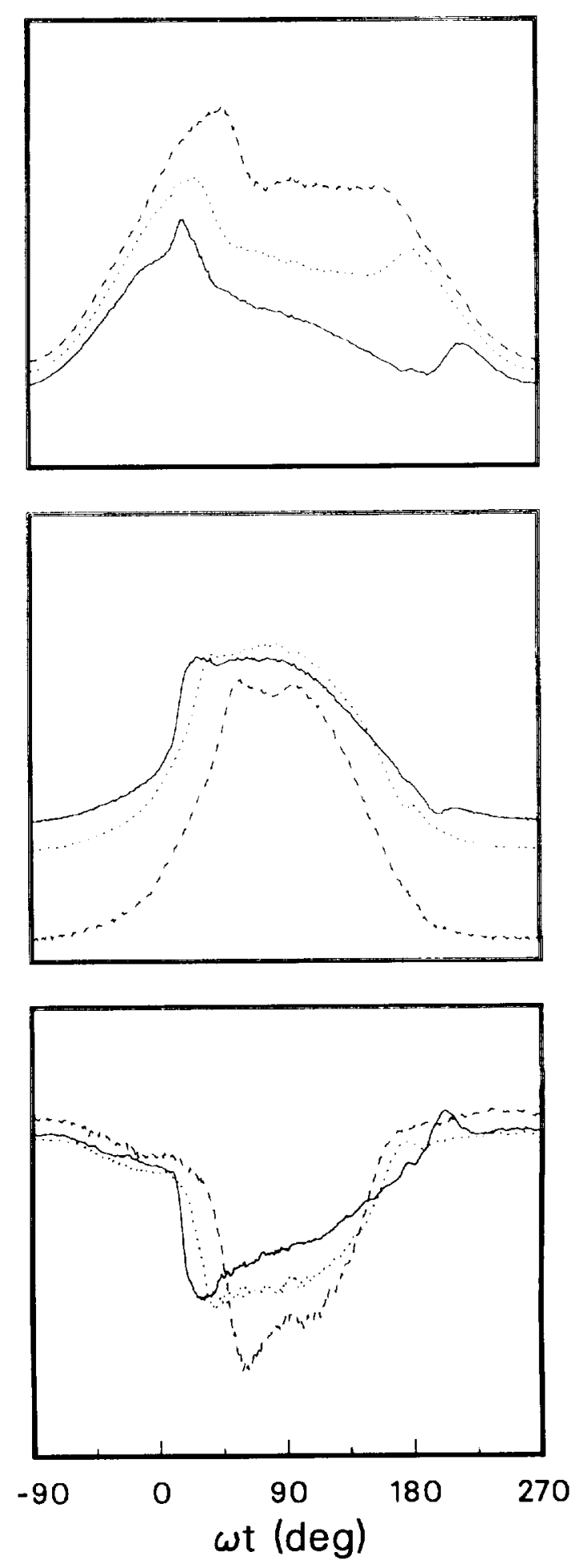

(b) Unsteady force and moment loads.

Figure 28. Concluded. 

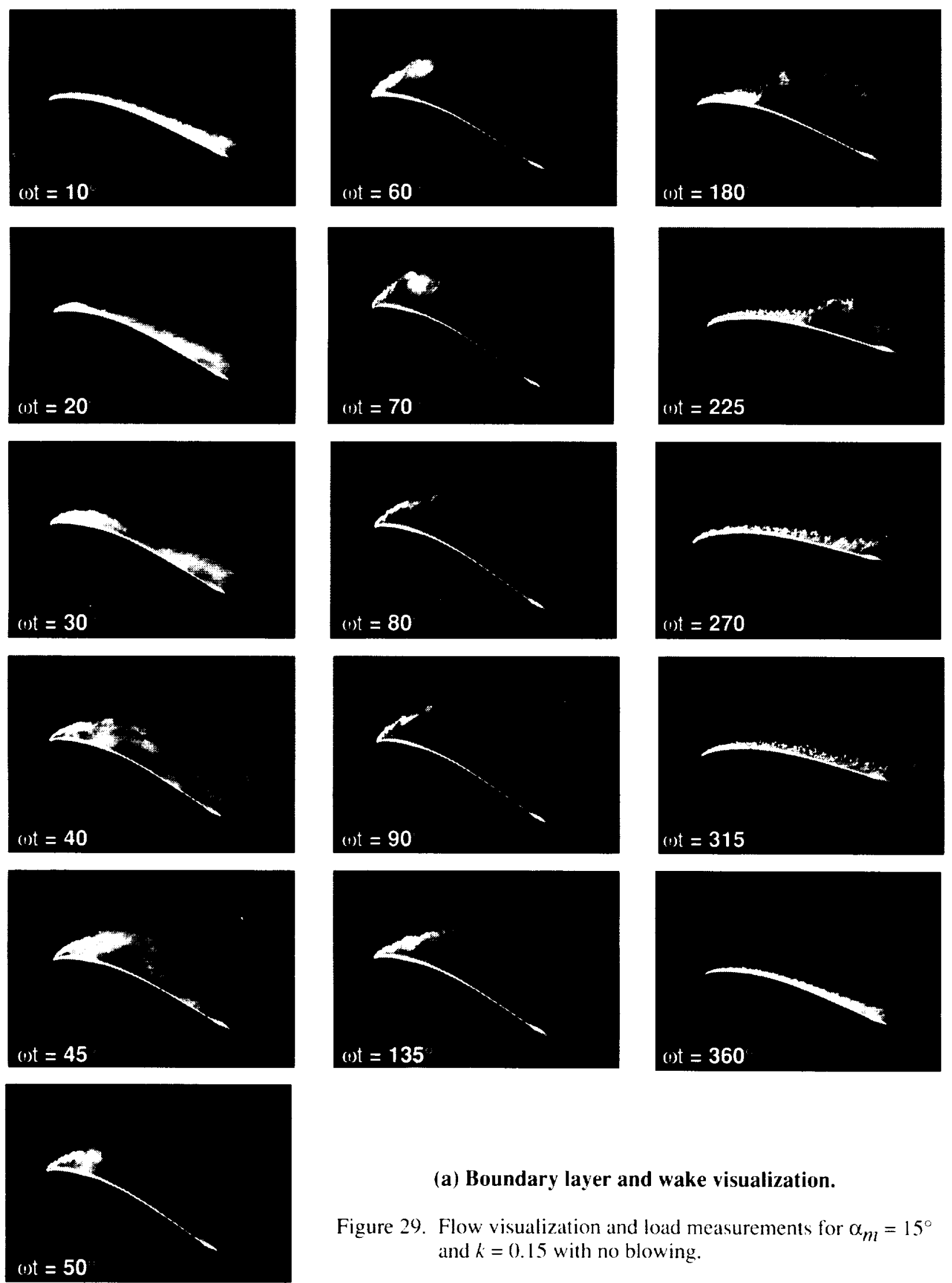

(a) Boundary layer and wake visualization.

Figure 29. Flow visualization and load measurements for $\alpha_{m}=15^{\circ}$ and $k=0.15$ with no blowing. 

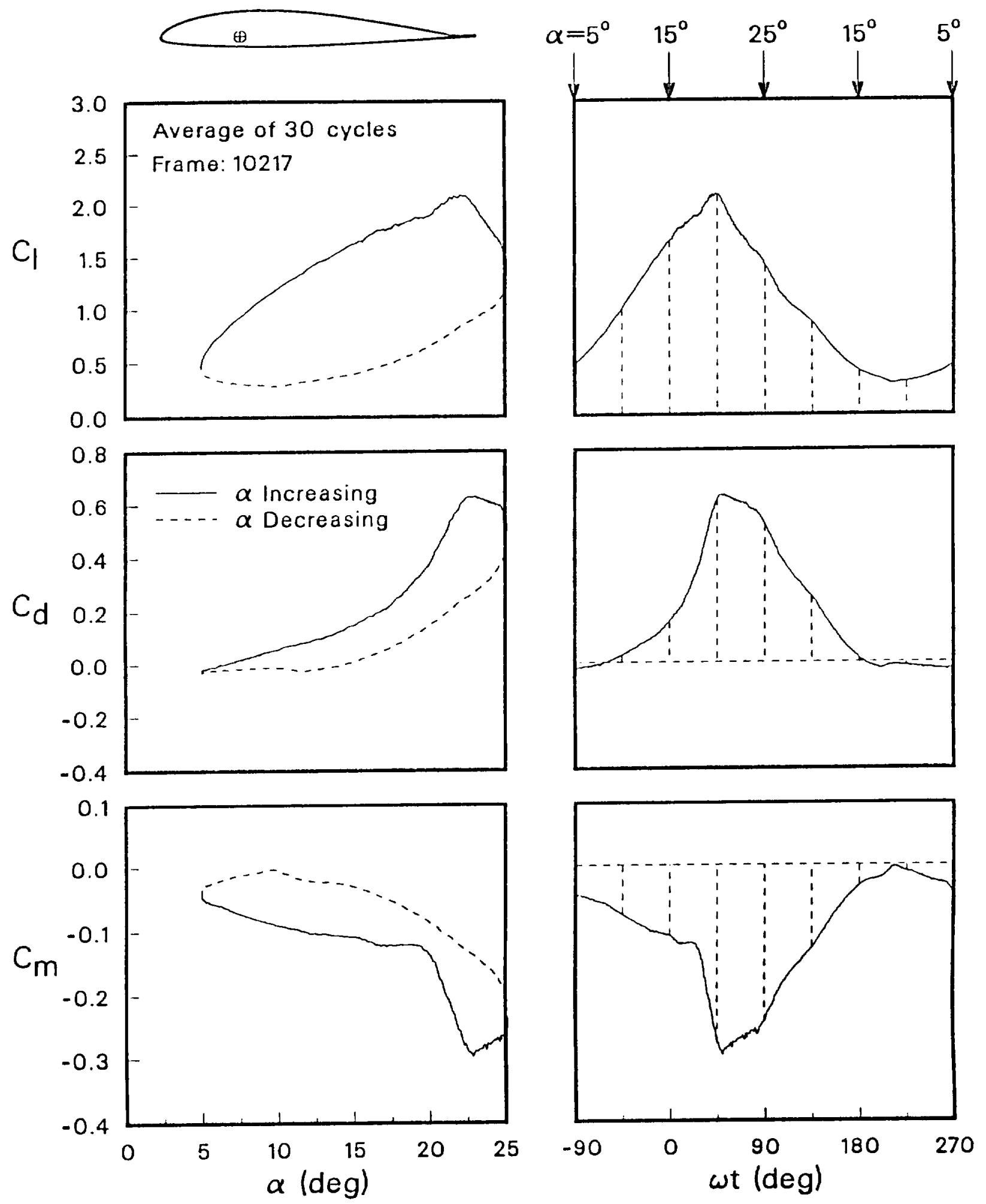

(b) Unsteady force and moment loads.

Figure 29. Concluded. 

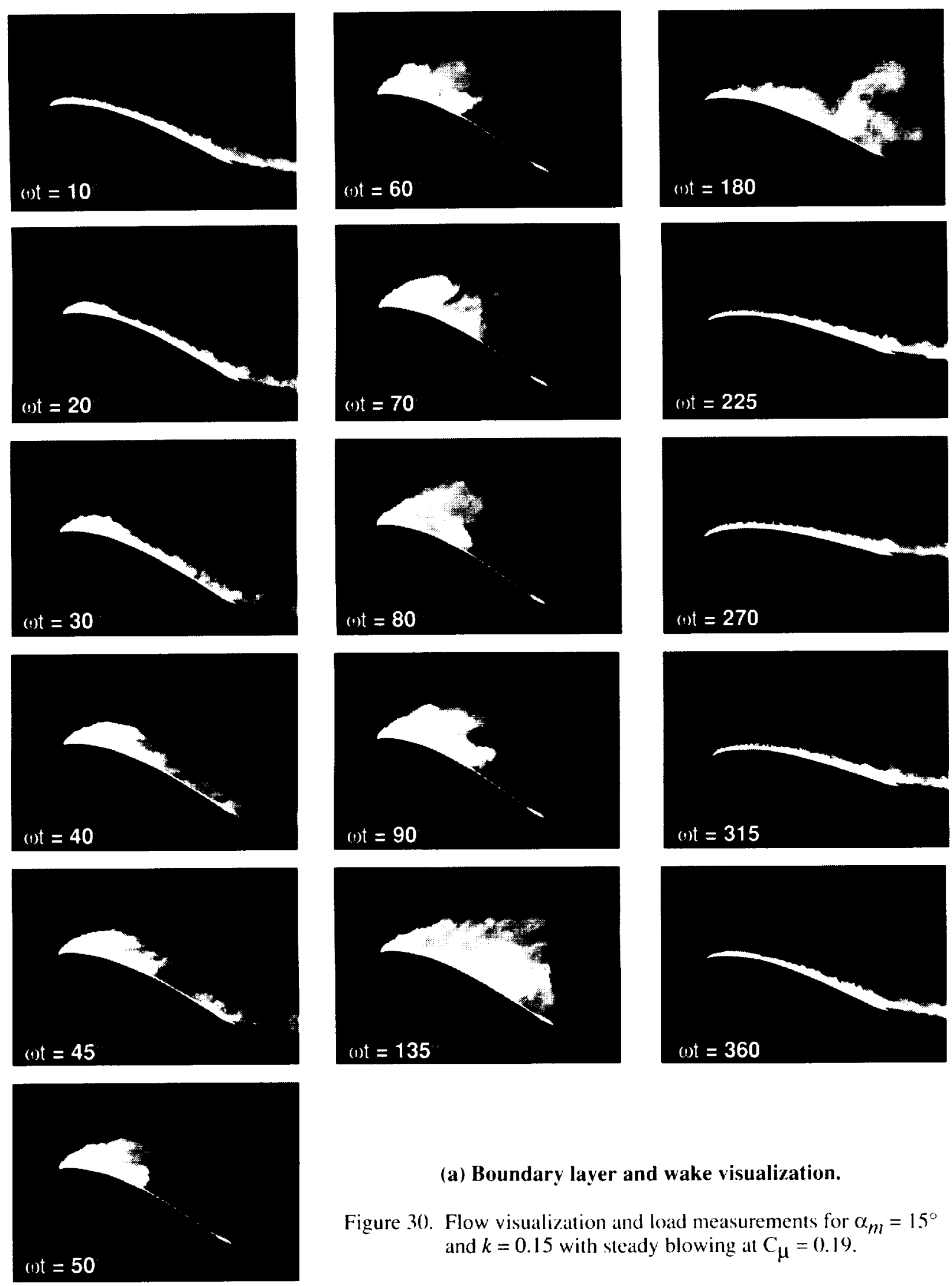

(a) Boundary layer and wake visualization.

Figure 30. Flow visualization and load measurements for $\alpha_{m}=15^{\circ}$ and $k=0.15$ with steady blowing at $C_{\mu}=0.19$. 

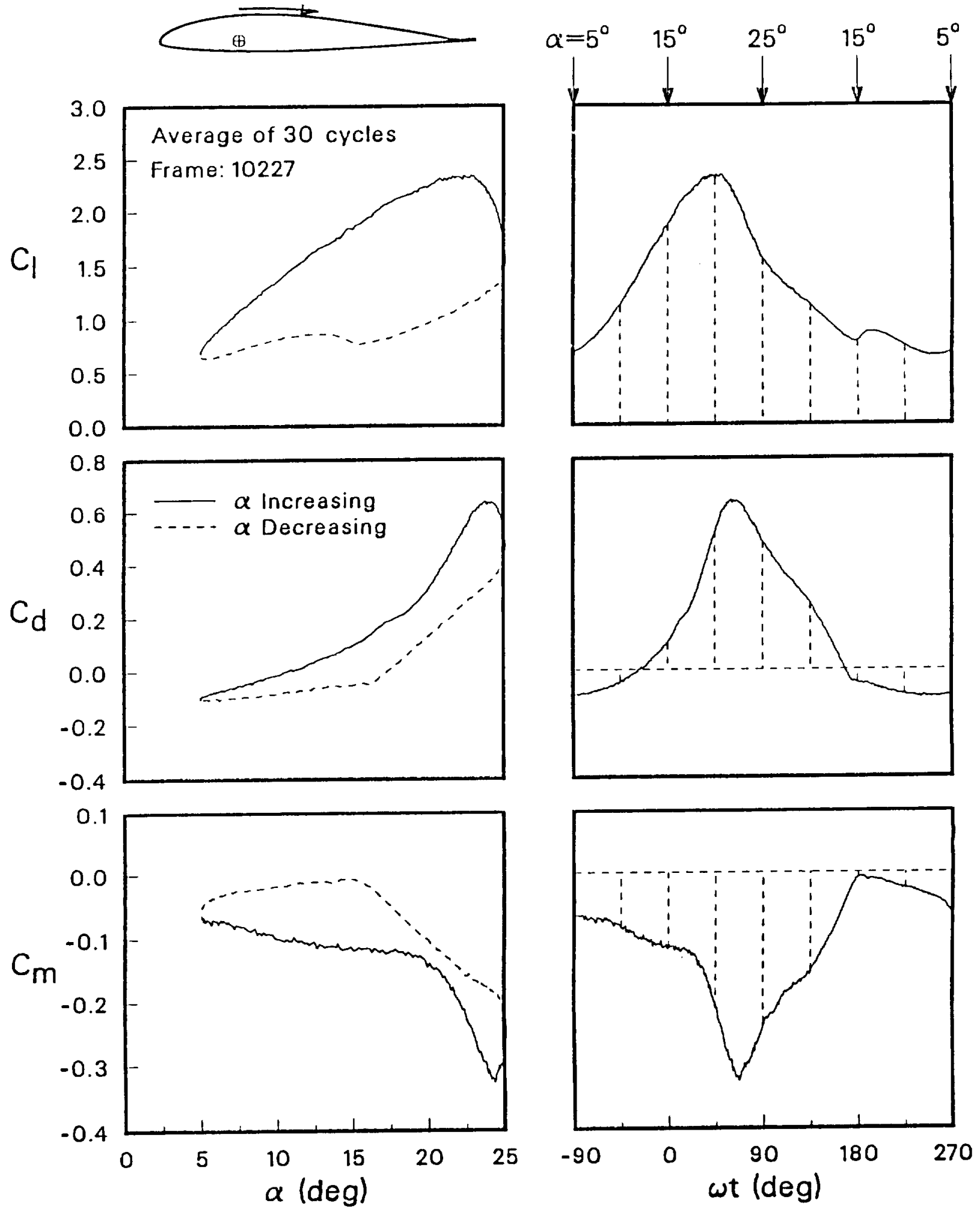

(b) Unsteady force and moment loads.

Figure 30. Concluded. 

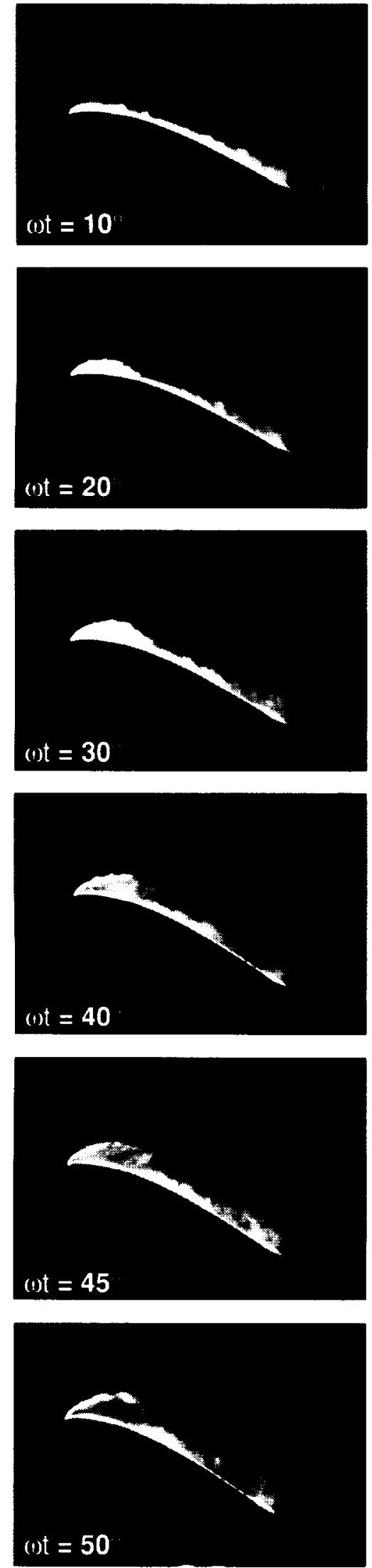
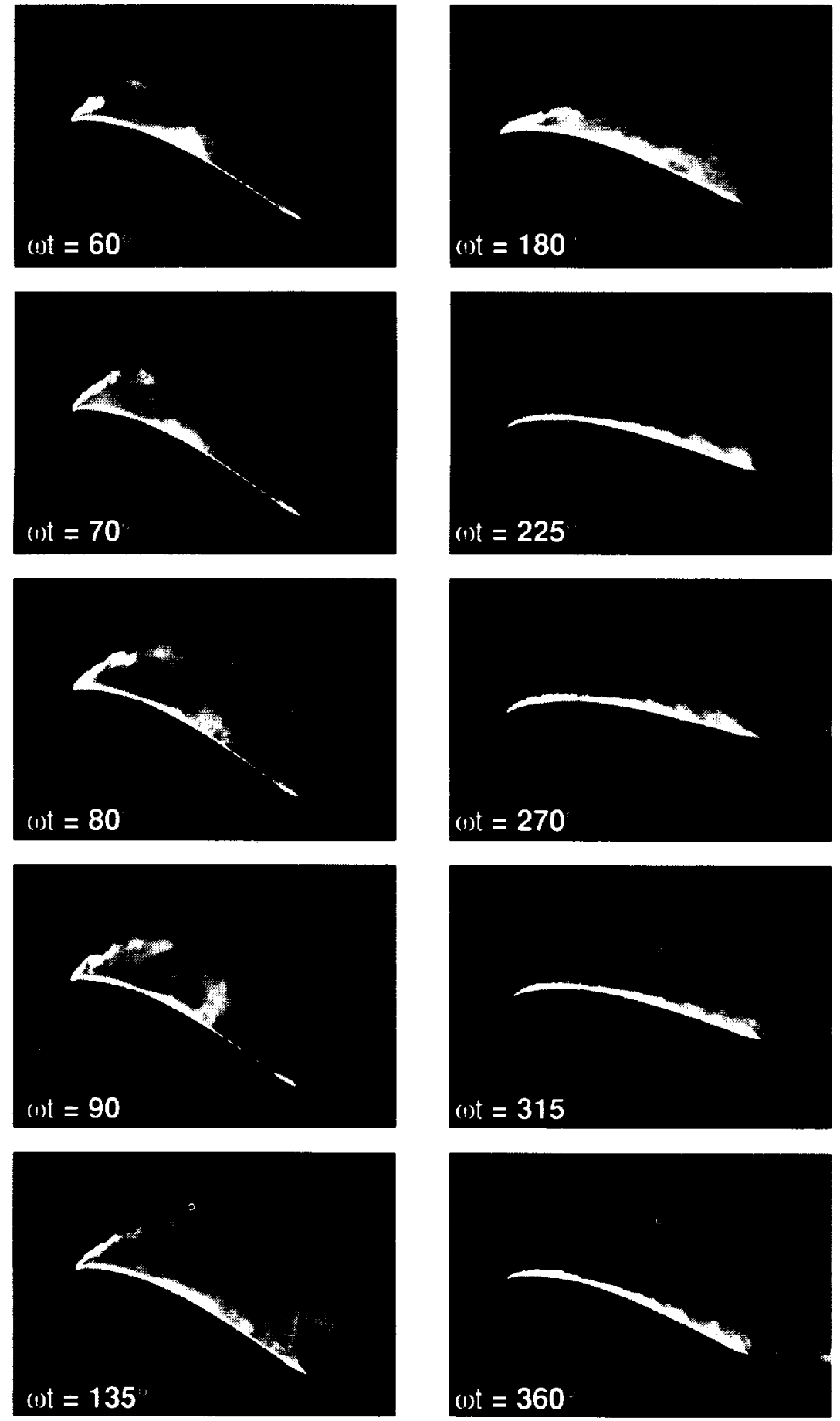

(a) Boundary layer and wake visualization.

Figure 31. Flow visualization and load measurements for $\alpha_{m}=15^{\circ}$ and $k=0.15$ with steady blowing at $C_{\mu}=0.65$. 
popnouos is ansty

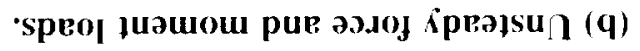

(Бәр) Im
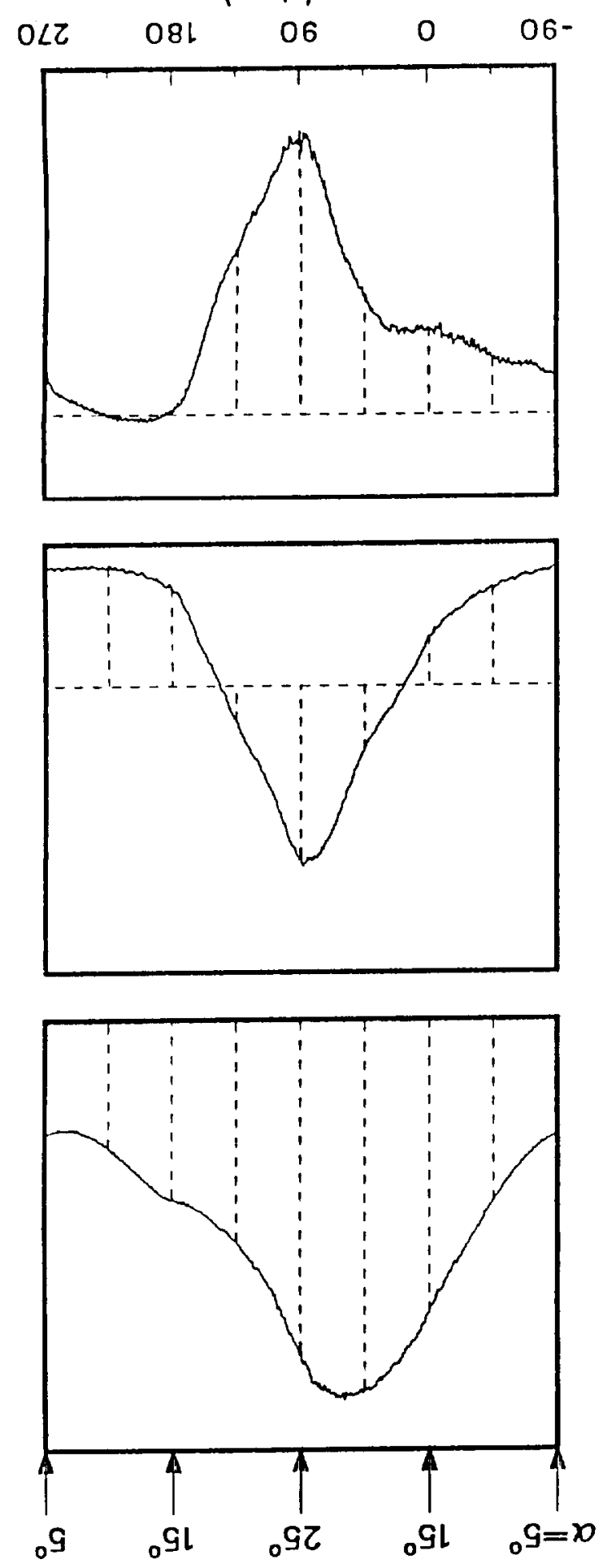

(6әр) 0

gz or $g l$ ol $g$ o
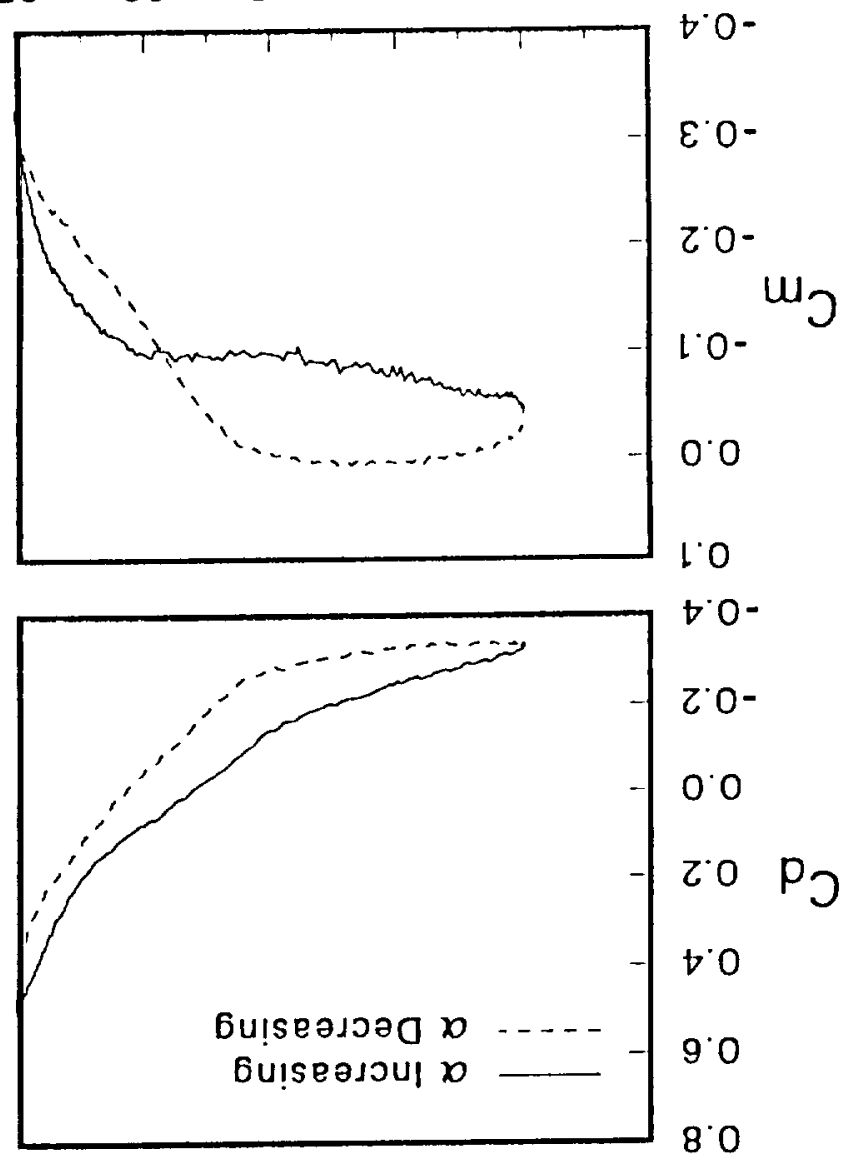

\section{†0-}

$2 \cdot 0-$

0.0

z०o po

$\forall \cdot 0$

$9 \cdot 0$

8.0

0.0

G.0

0.1

9110

$0 \cdot z$

$\mathbf{G}^{\prime} \mathbf{Z}$

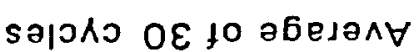
$0^{\circ} \varepsilon$ 

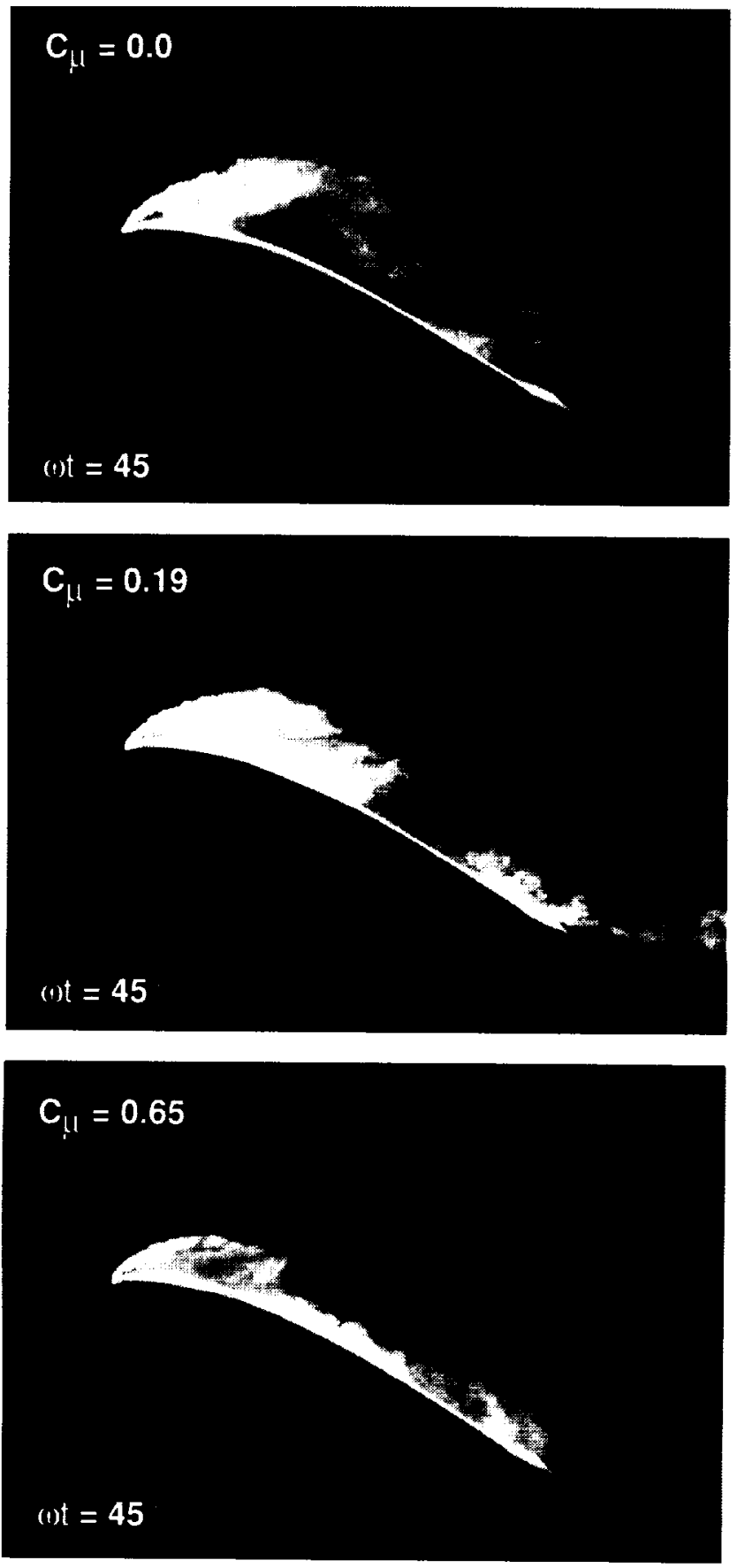

(a) Boundary layer and wake visualization.

Figure 32. Flow visualization and load measurements for $\alpha_{m}=15^{\circ}$ and $k=0.15$ at $\omega \mathrm{t}=45^{\circ}\left(\alpha=22^{\circ}\right)$ with different blowing rates. 


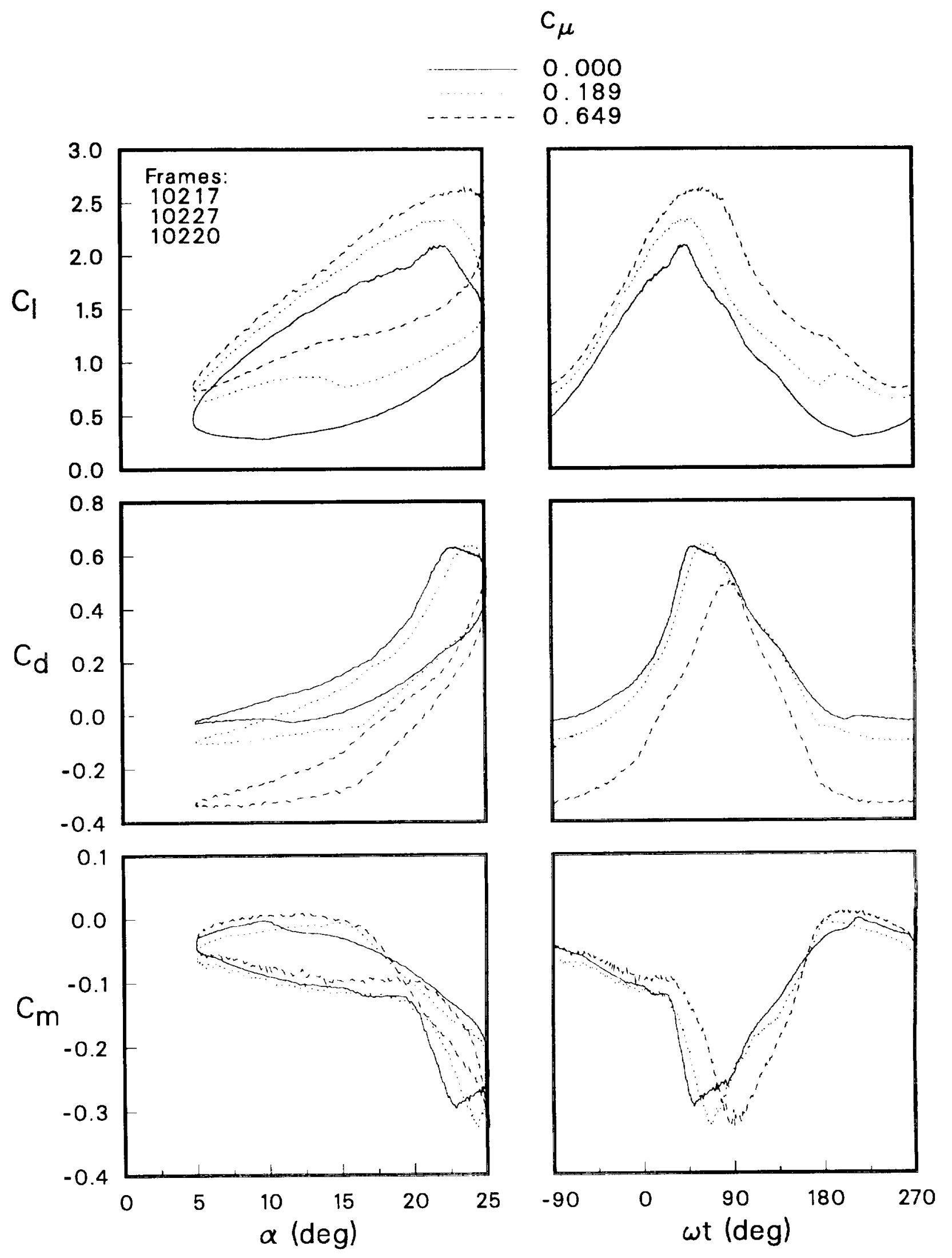

(b) Unsteady force and moment loads.

Figure 32. Concluded. 
0.005

0.052

o. 150
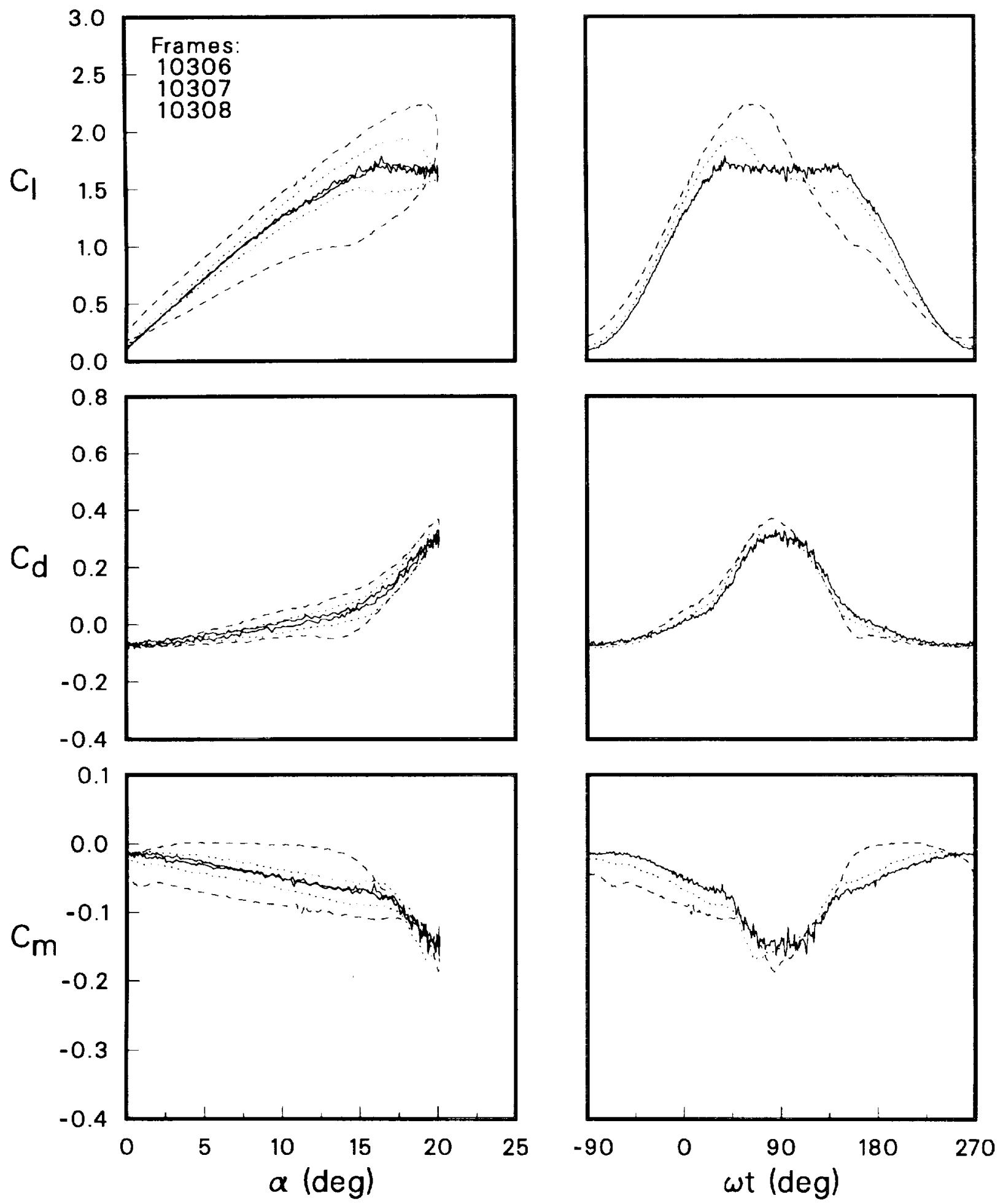

Figure 33. Effects of reduced frequency with $C_{\mu}=0.16$ for $\alpha_{m}=10^{\circ}$. 


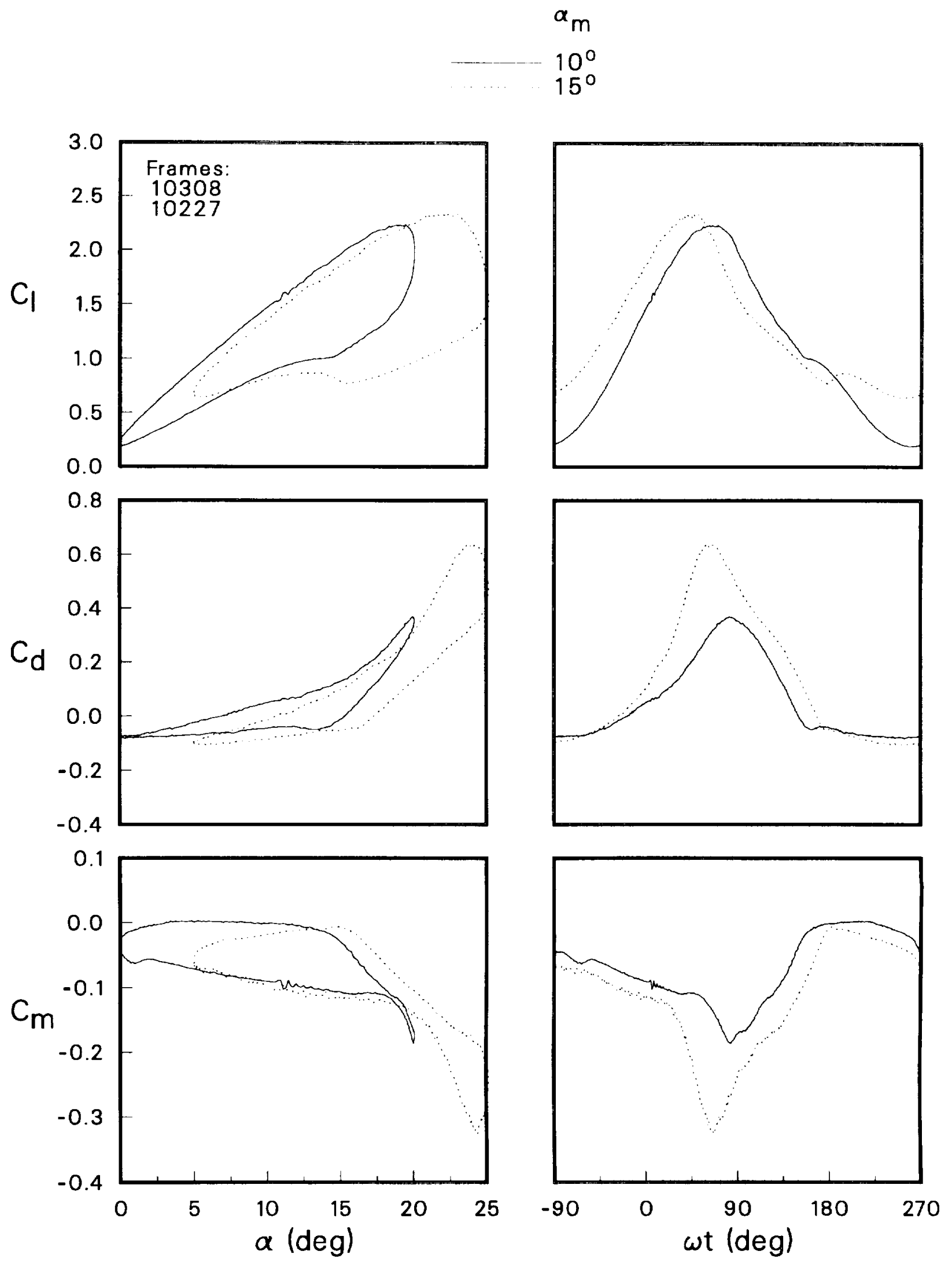

Figure 34. Effects of mean angle with $\mathrm{C}_{\mu}=0.18$ and $k=0.15$. 

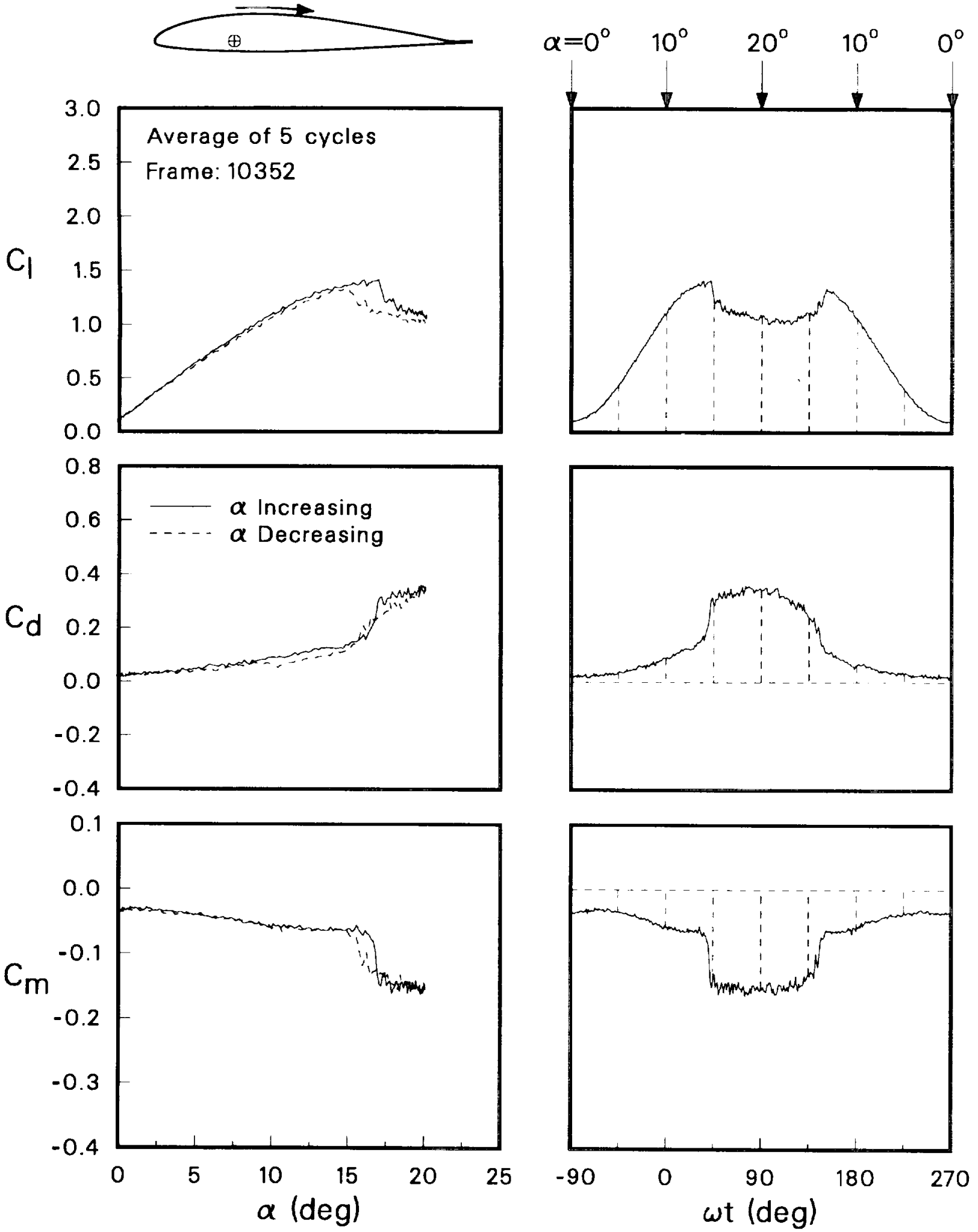

Figure 35. Unsteady loads for $\alpha_{m}=10^{\circ}$ at $k=0.006$ with steady blowing at $C_{\mu}=0.023$. 


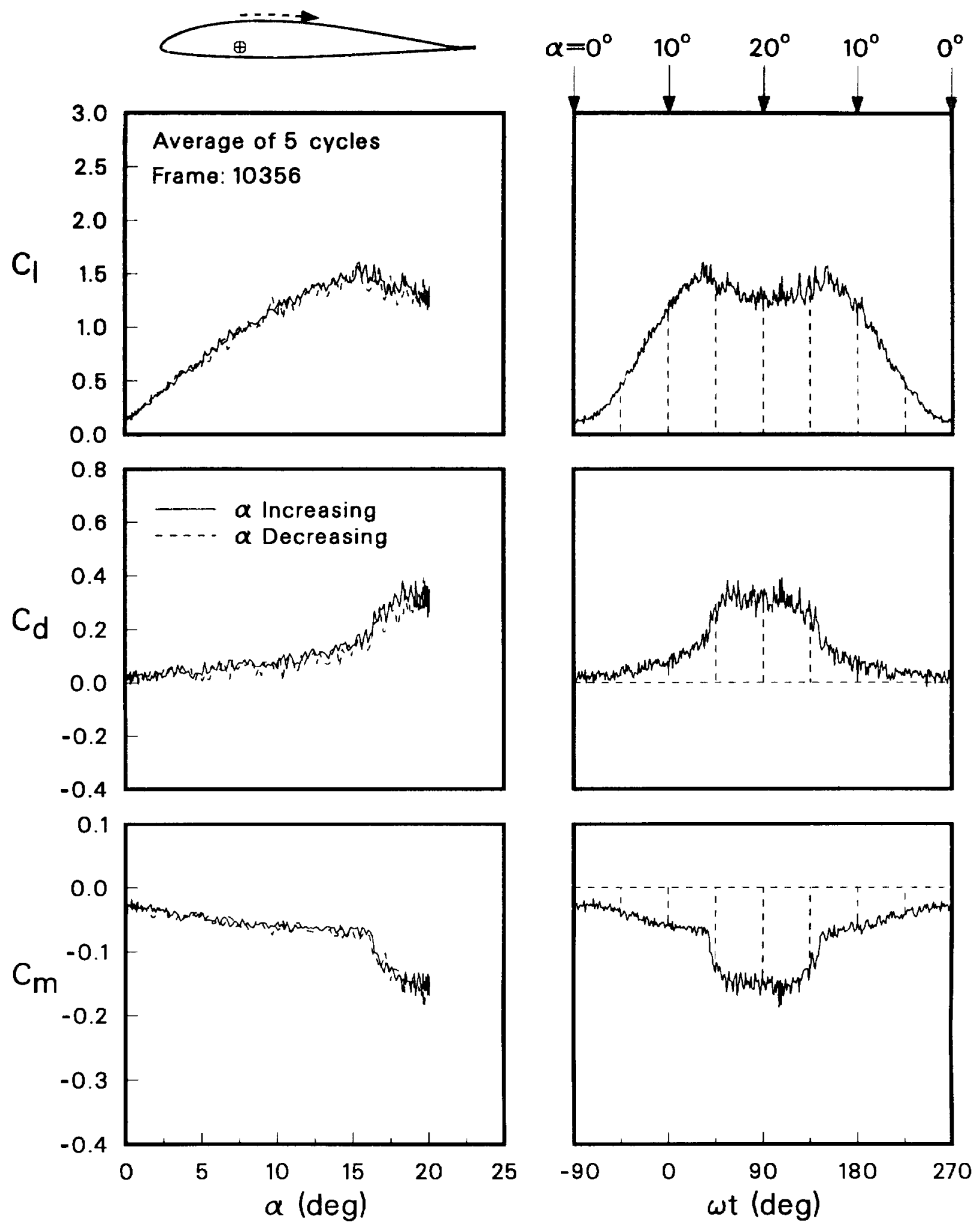

Figure 36. Unsteady loads for $\alpha_{m}=10^{\circ}$ at $k=0.005$ with unsteady blowing at $\mathrm{C}_{\mu}=0.024$ and $F^{+}=0.9$. 


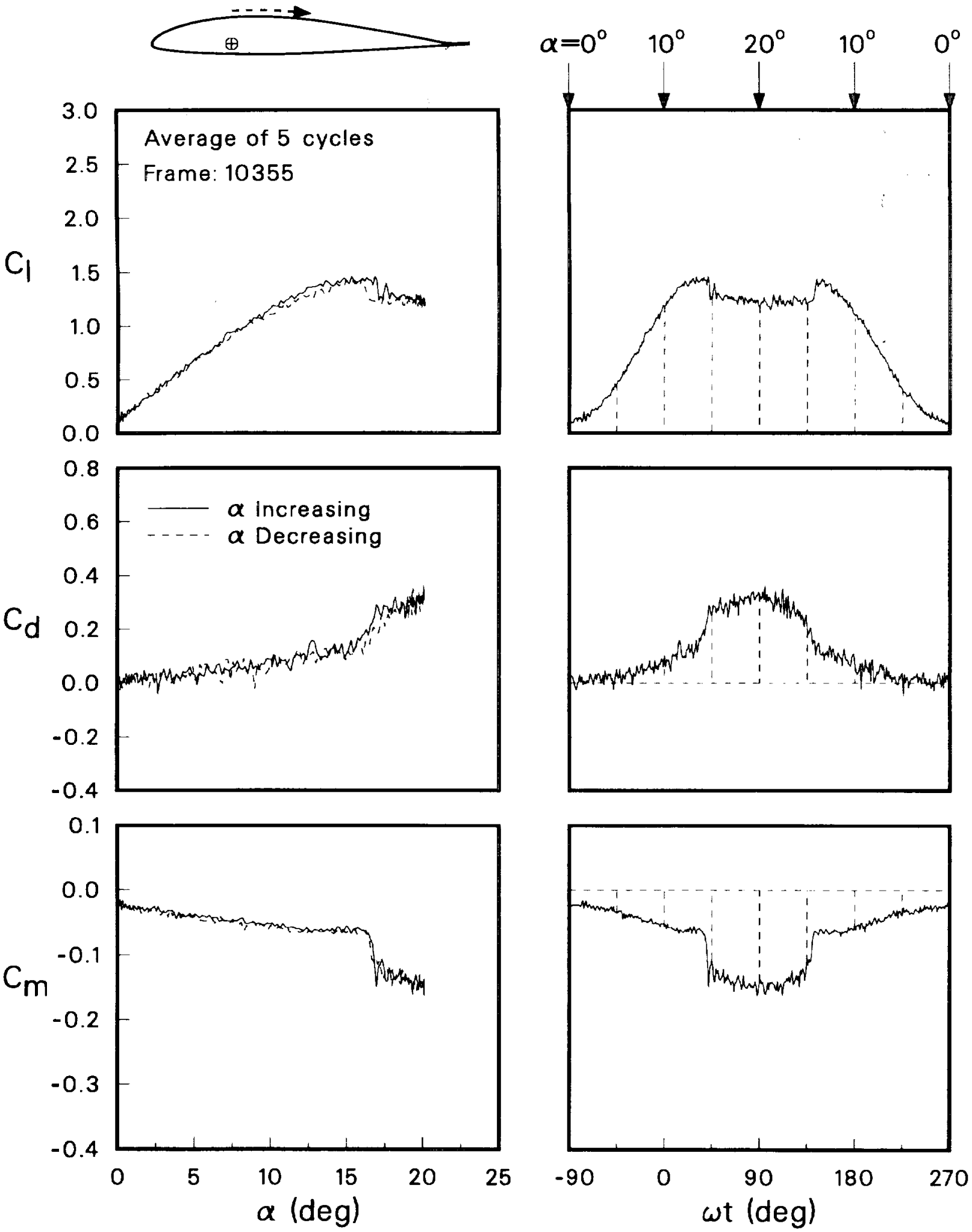

Figure 37. Unsteady loads for $\alpha_{m}=10^{\circ}$ at $k=0.006$ with unsteady blowing at $\mathrm{C}_{\mu}=0.023$ and $F^{+}=1.9$. 


$$
L \cdot z=+t \text { pue } t z 0 \cdot 0=H
$$

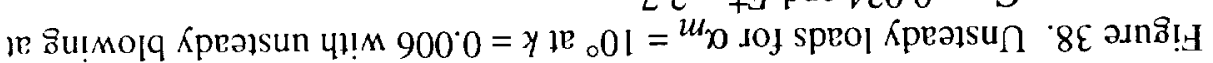

(6әр) $1 m$
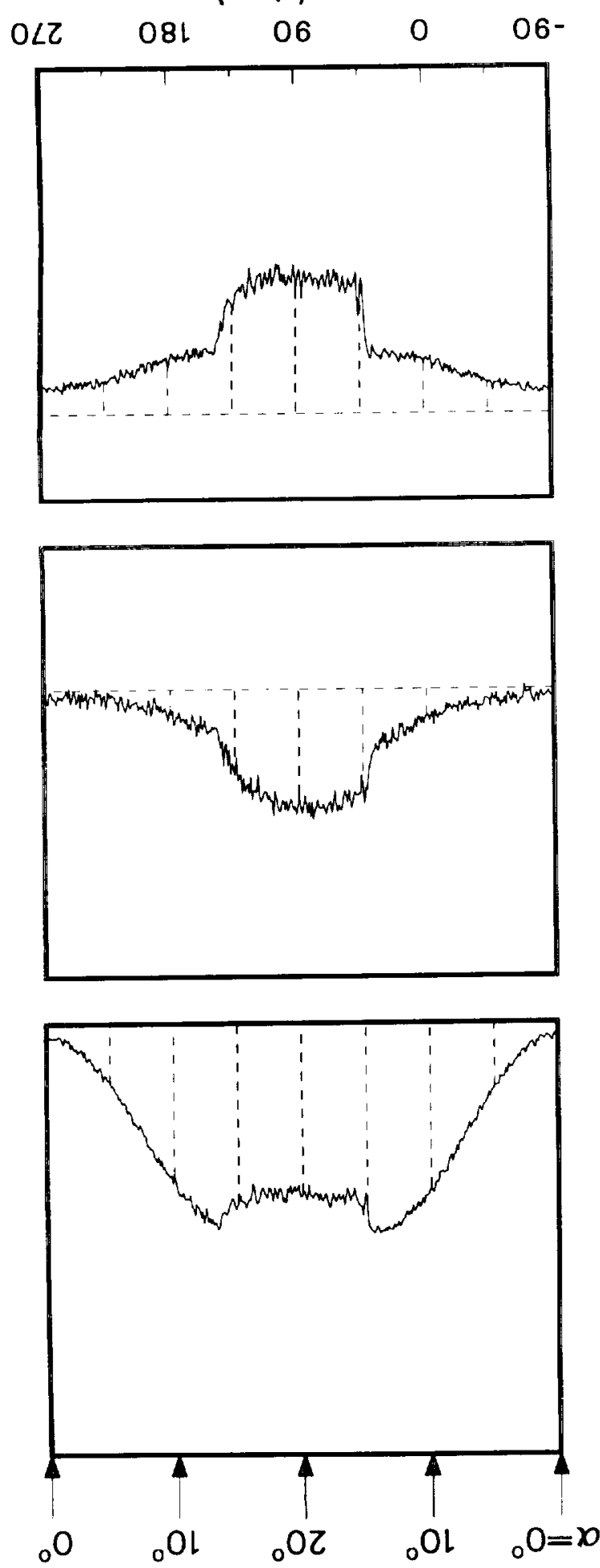

(6әр) 0

gz oz gl ol 9 o

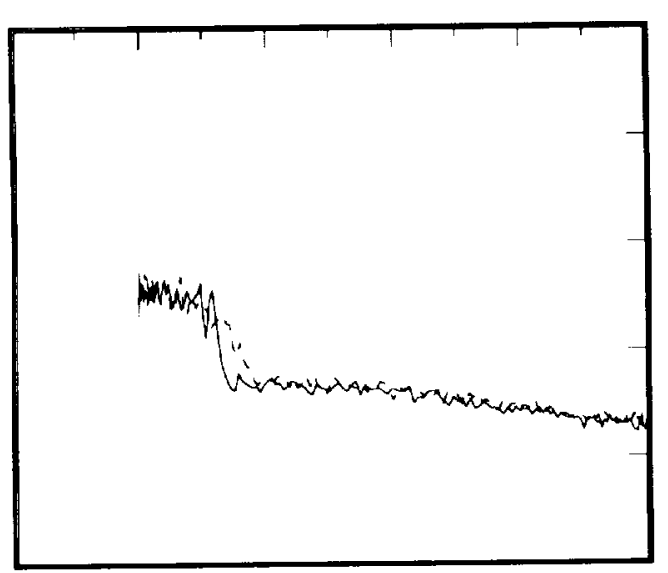

to-

$\varepsilon^{\prime} 0^{-}$

$20-$

แว

เ.O-

0.0

10

to-

$20-$

0.0

$2 \cdot 0$

Po

to

Gu!seajoad do .....

6ulseasoul $x$

$9 \cdot 0$

$8^{\circ} 0$

0.0

$9 \cdot 0$

0.1

9. b

0.2

$\angle 98 O L$ : өmest

$9 \cdot 2$

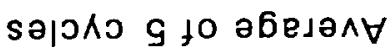

$0 \cdot \varepsilon$ 


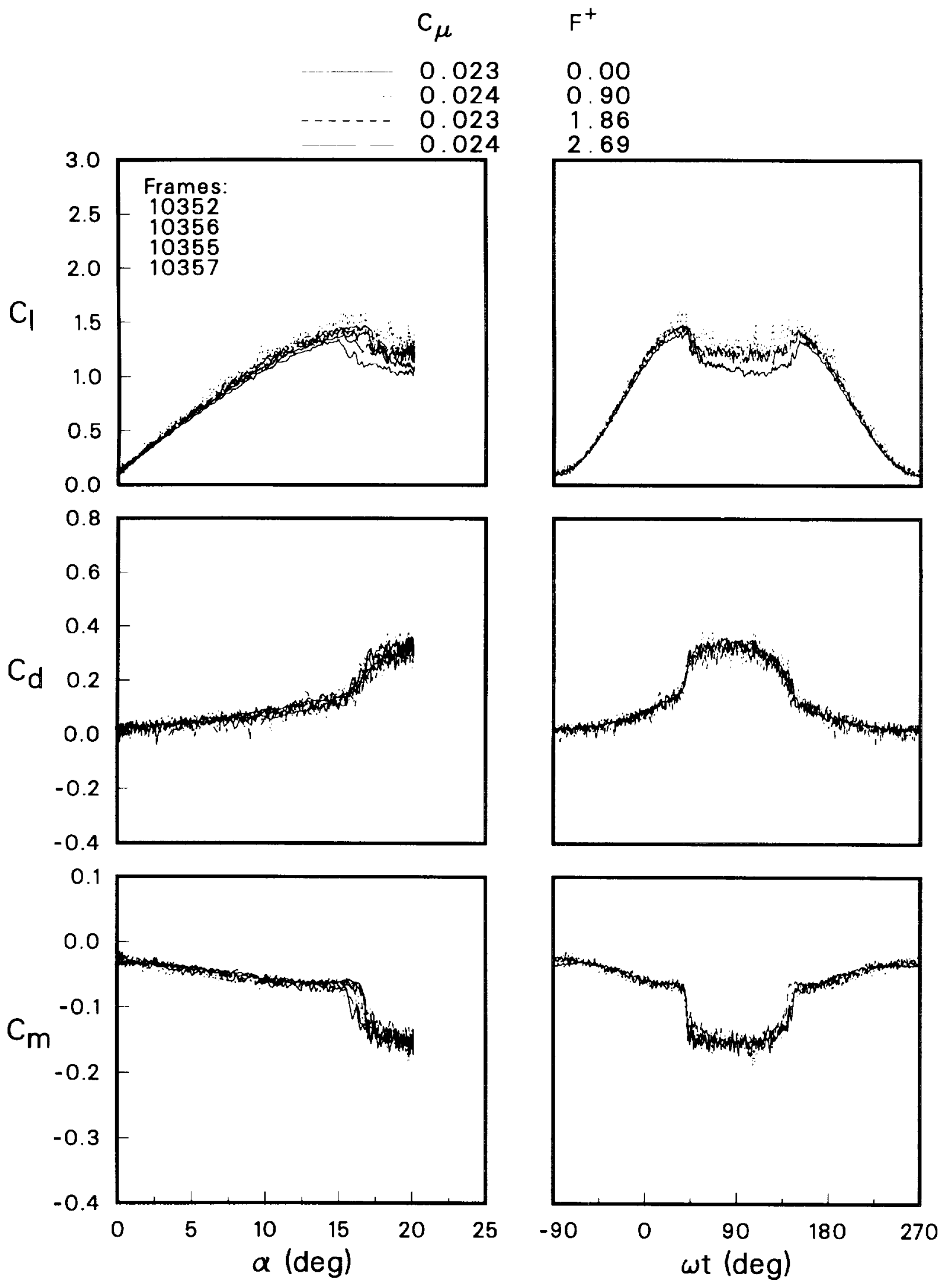

Figure 39. Effects of unsteady blowing for $\alpha_{m}=10^{\circ}$ and $k=0.006$. 


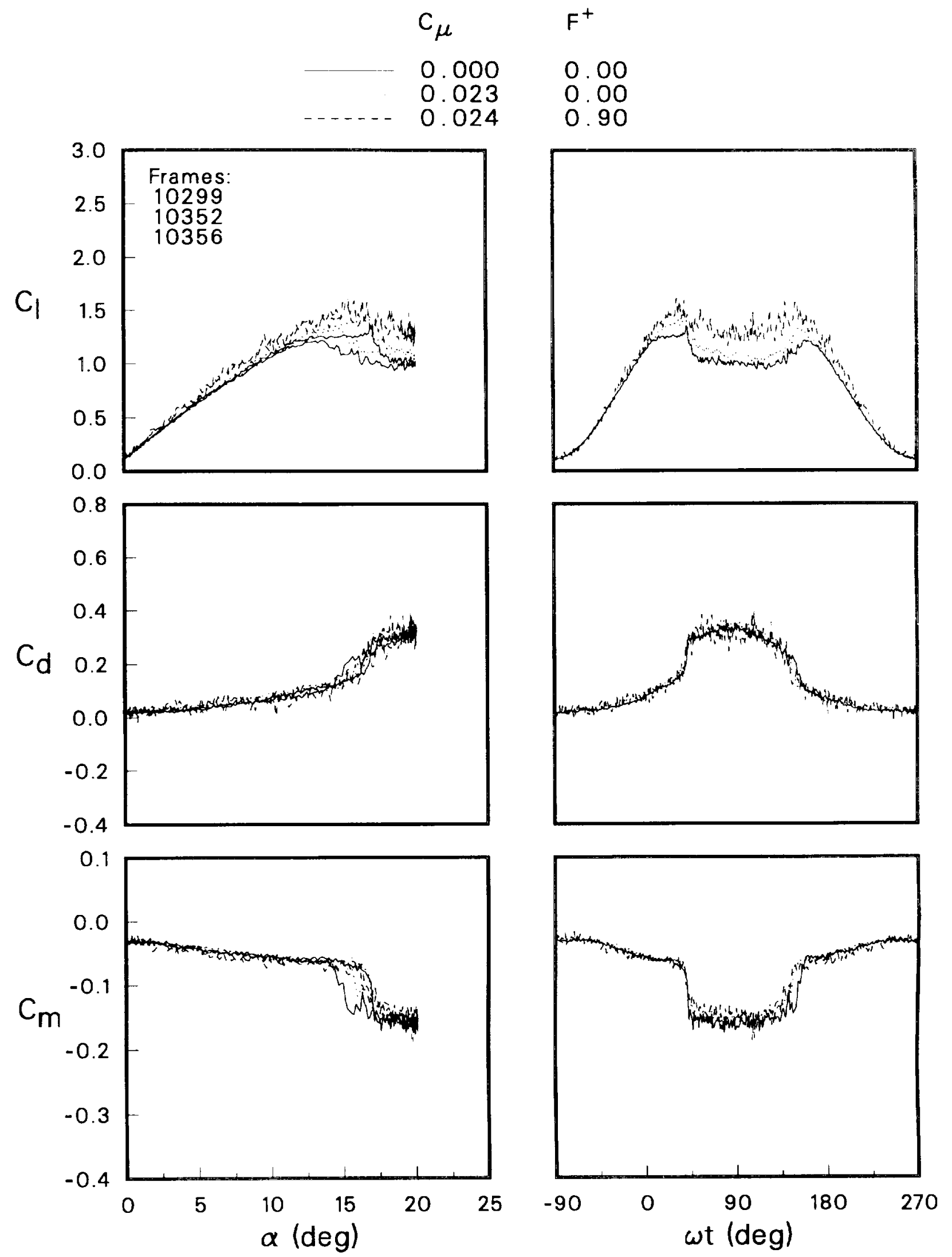

Figure 40. Effects of steady and unsteady blowing for $\alpha_{m}=10^{\circ}$ and $k=0.005$. 

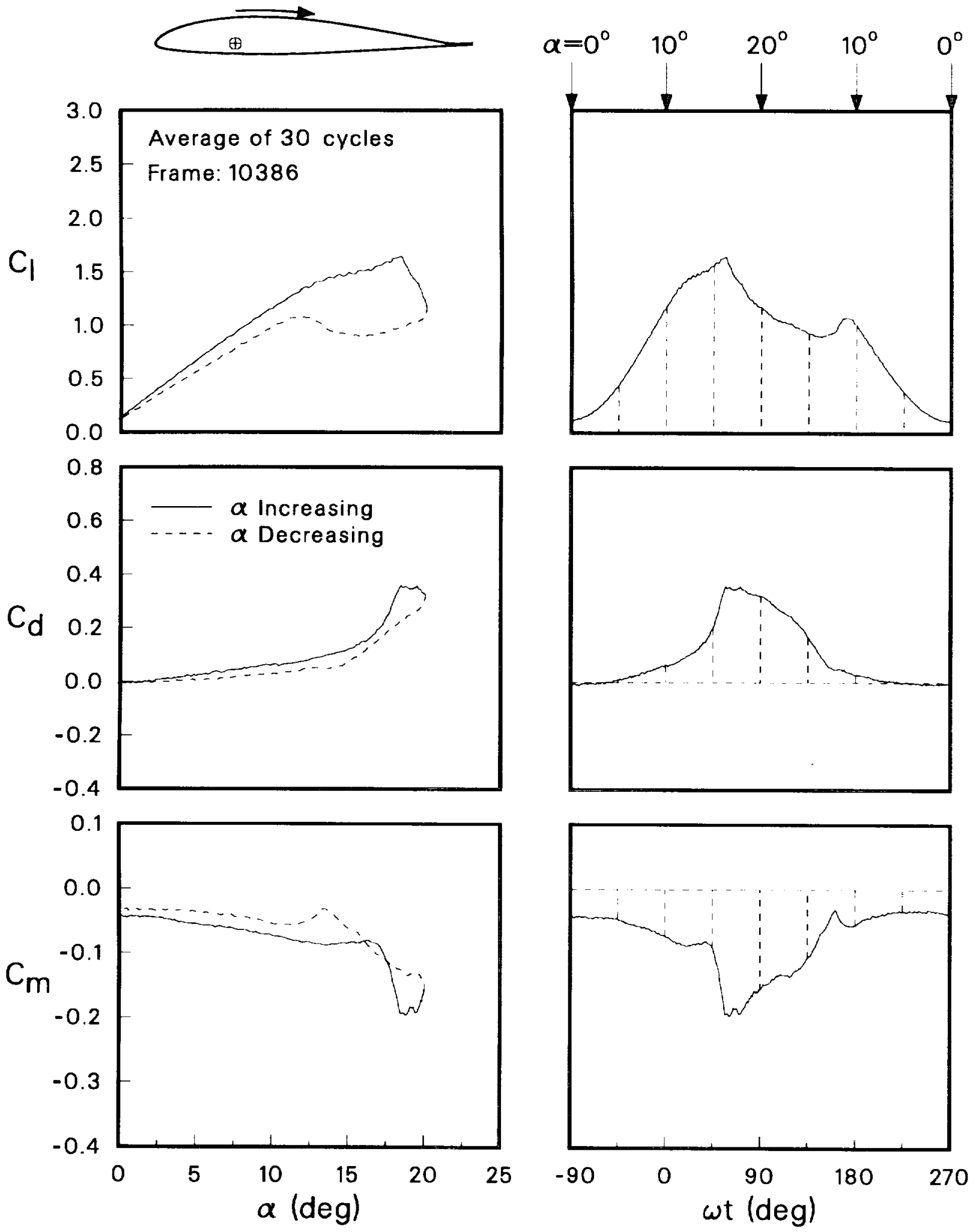

Figure 41. Unsteady loads for $\alpha_{m}=10^{\circ}$ and $k=0.05$ with steady blowing at $C_{\mu}=0.025$. 

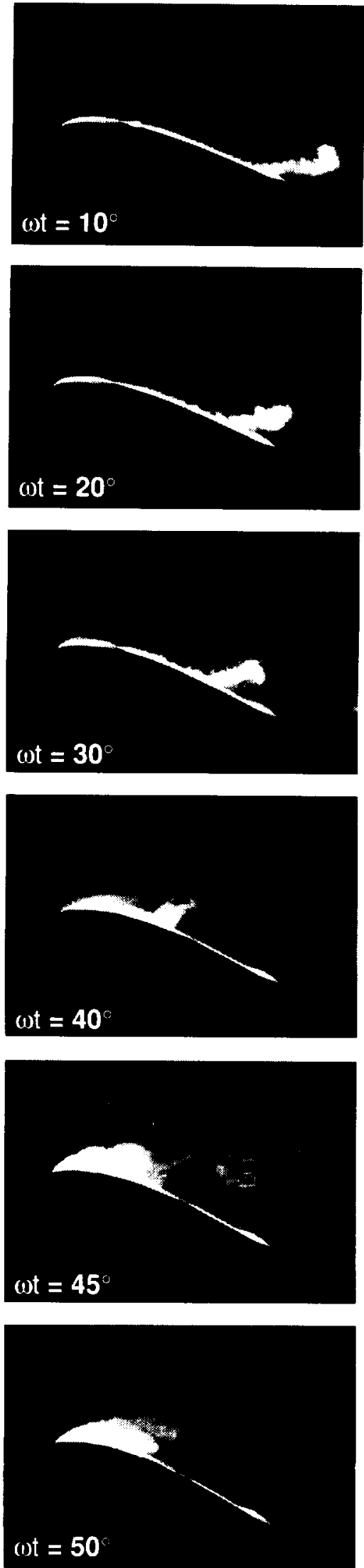
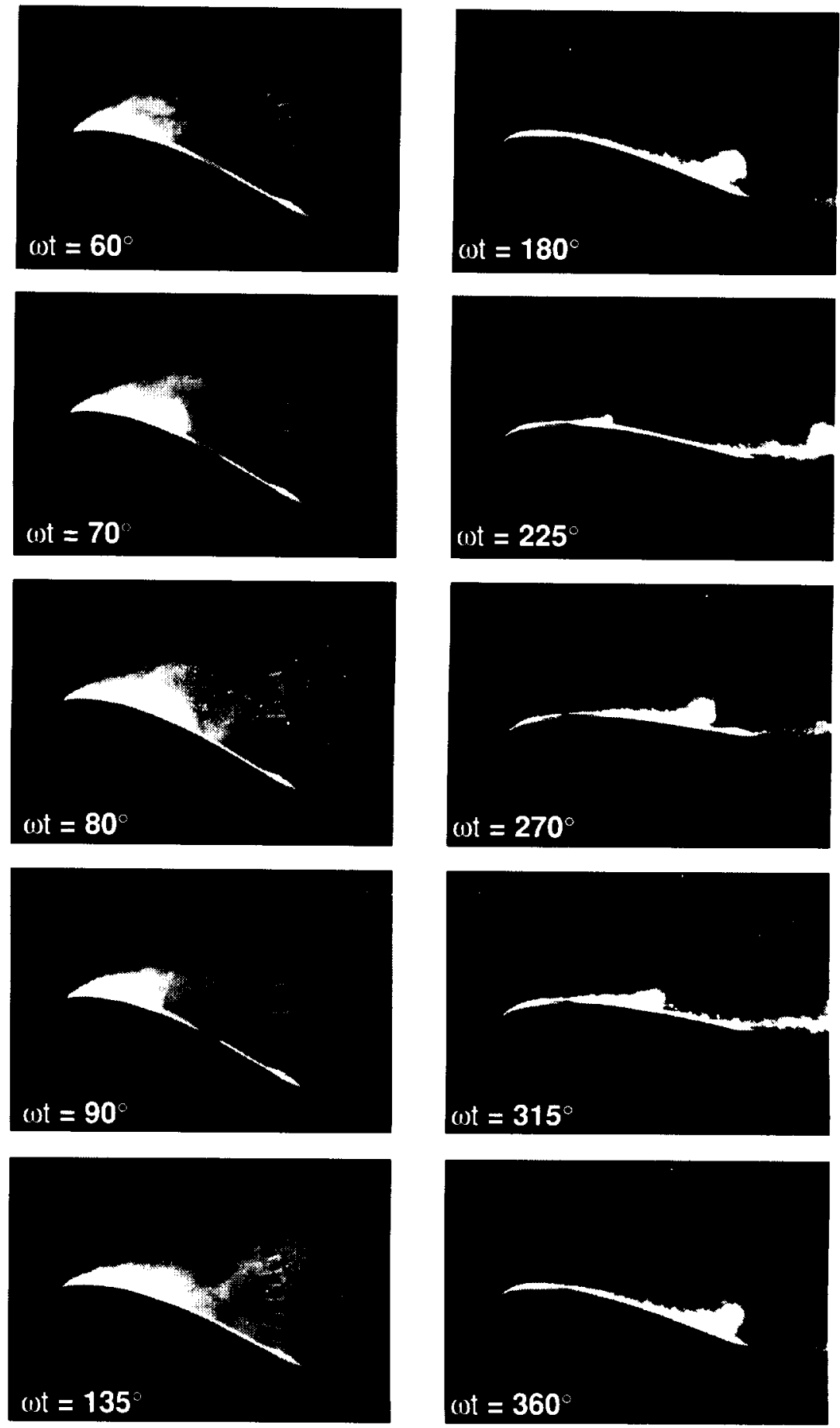

(a) Boundary layer and wake visualization.

Figure 42. Flow visualization and load measurements for $\alpha_{m}=10^{\circ}$ and $k=0.05$ with unsteady blowing at $\mathrm{C}_{\mu}=0.024$ and $F^{+}=0.9$. 

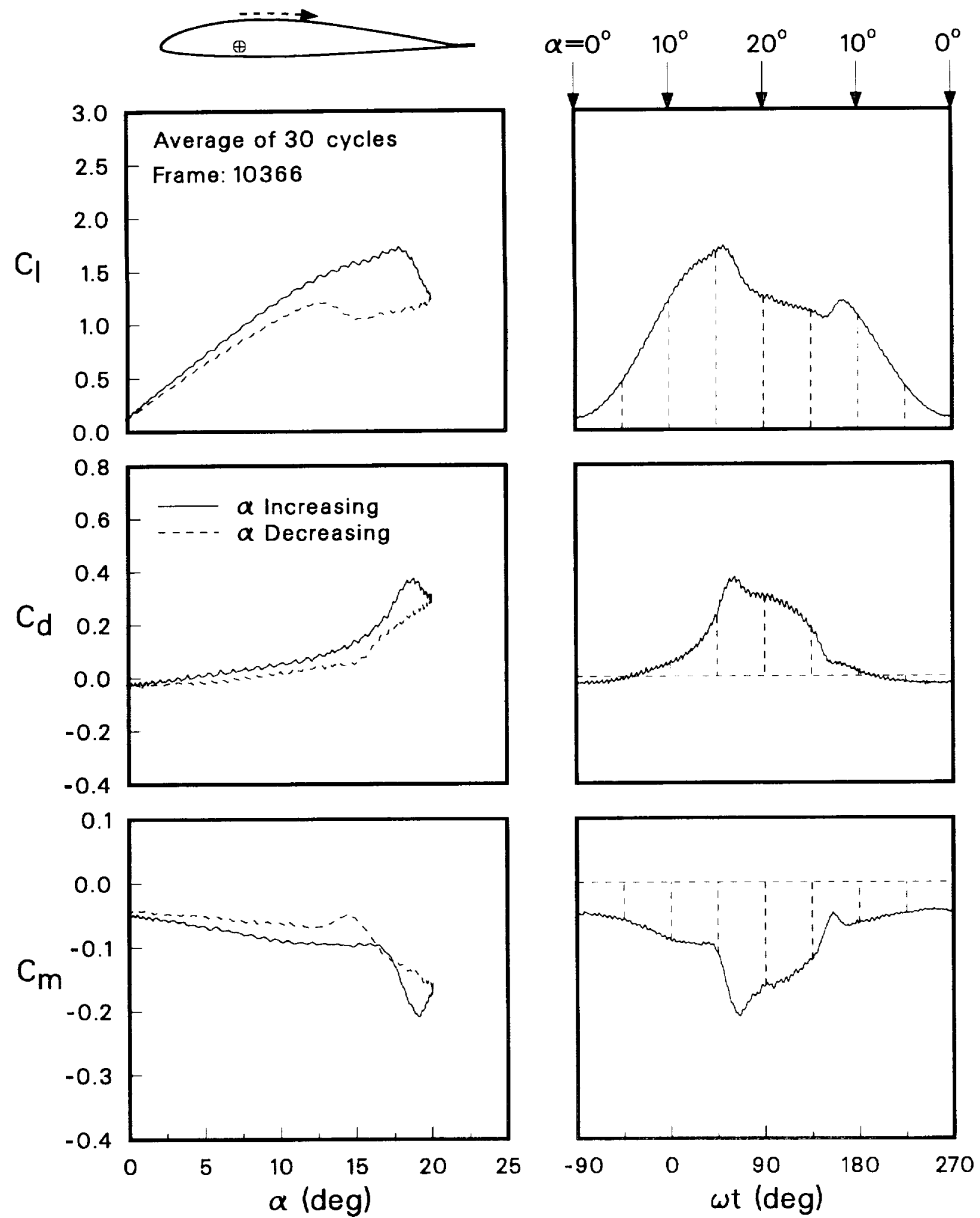

(b) Unsteady force and moment loads.

Figure 42. Concluded. 




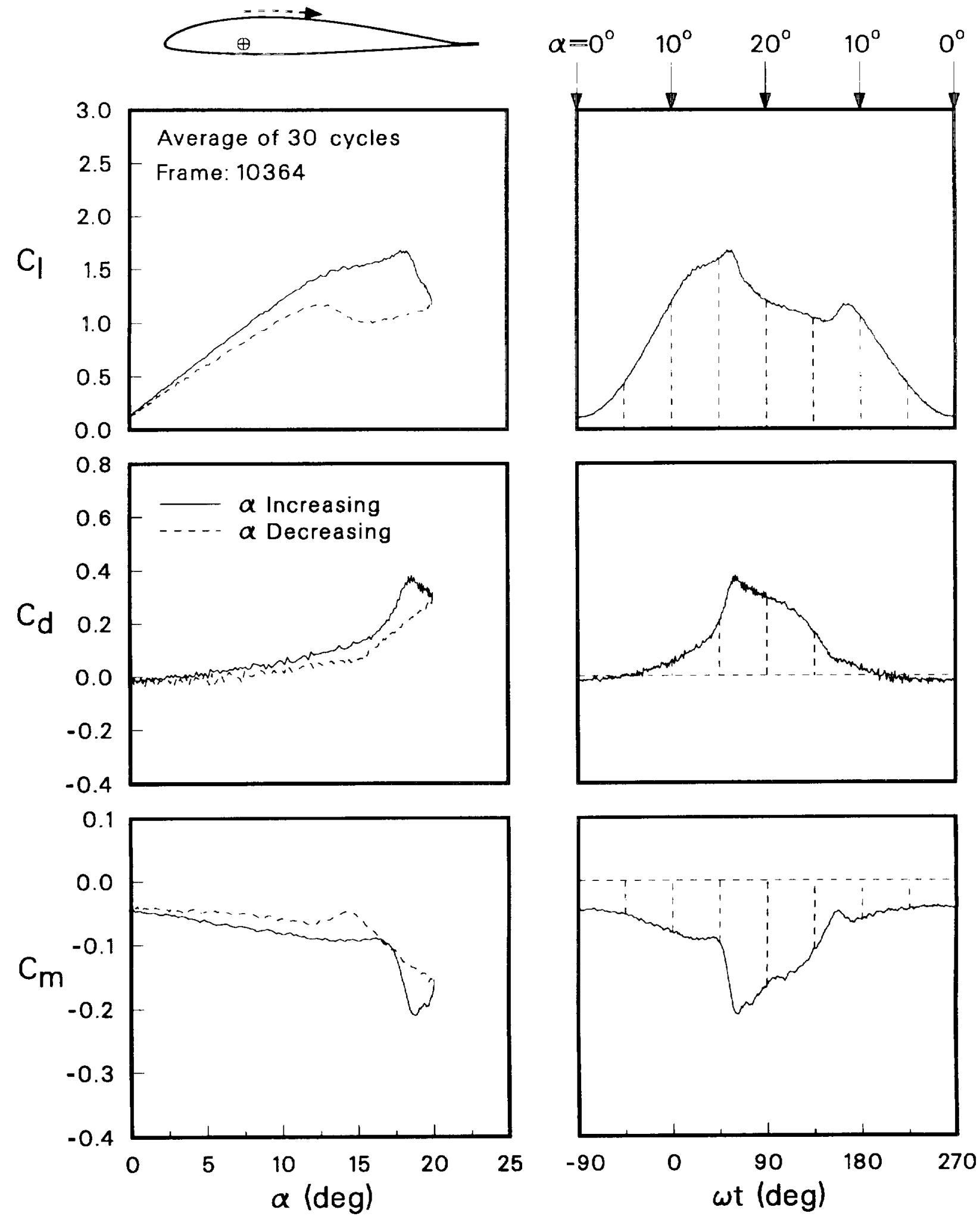

Figure 43. Unsteady loads for $\alpha_{m}=10^{\circ}$ and $k=0.05$ with unsteady blowing at $\mathrm{C}_{\mu}=0.023$ and $F^{+}=1.9$. 

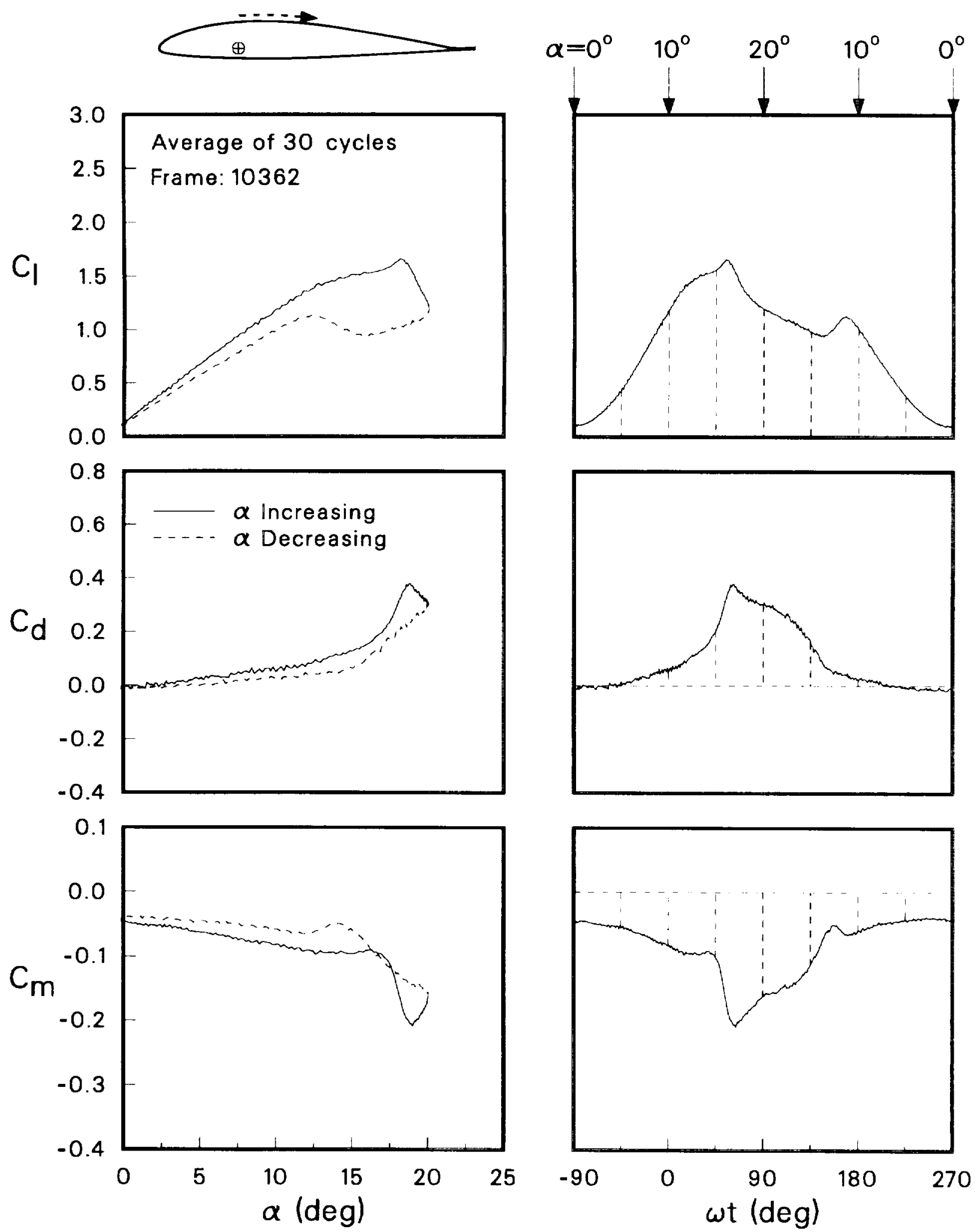

Figure 44. Unsteady loads for $\alpha_{m}=10^{\circ}$ and $k=0.05$ with unsteady blowing at $\mathrm{C}_{\mu}=0.024$ and $F^{+}=2.7$. 


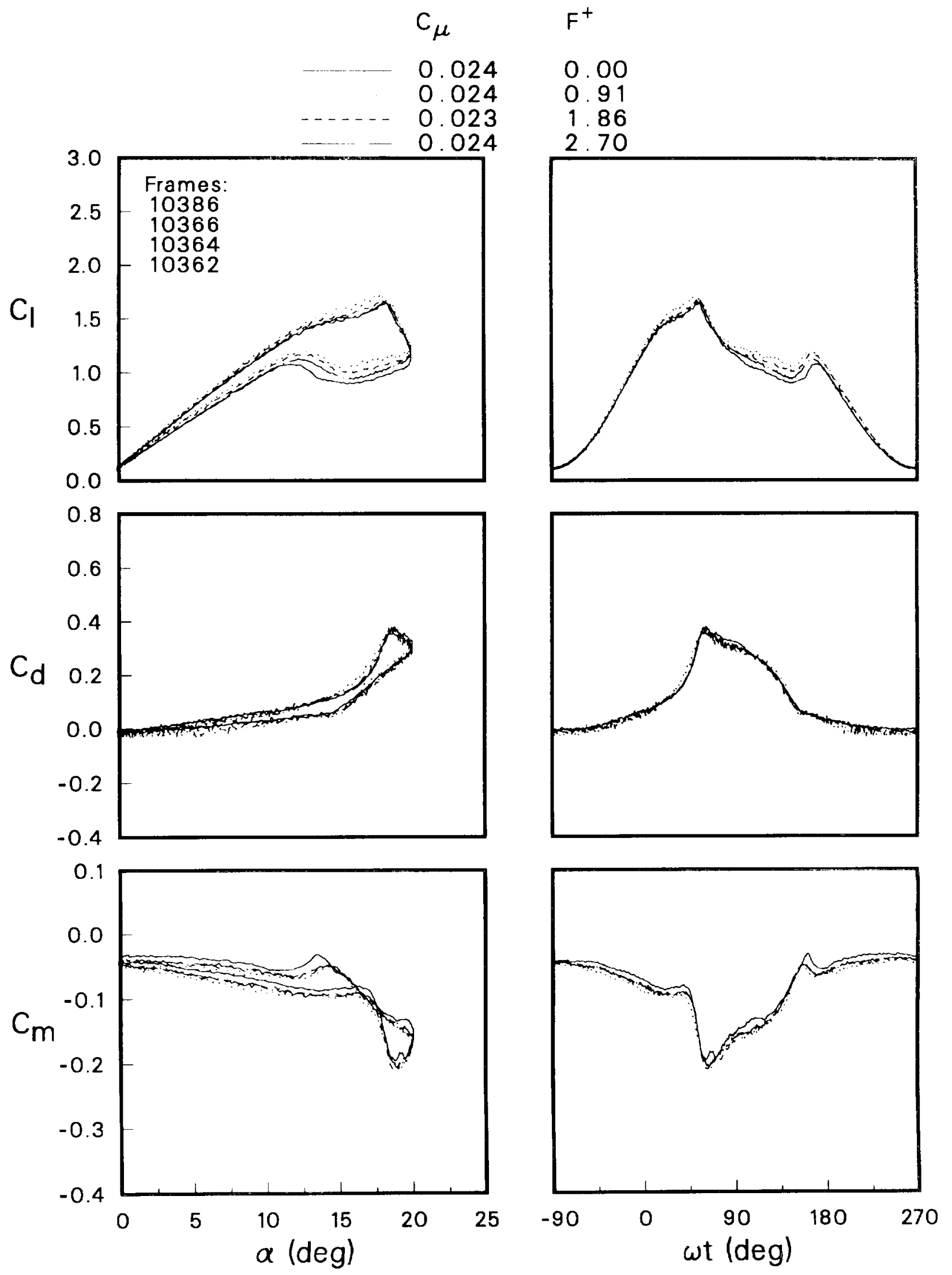

Figure 45. Effects of unsteady blowing for $\alpha_{m}=10^{\circ}$ and $k=0.05$. 

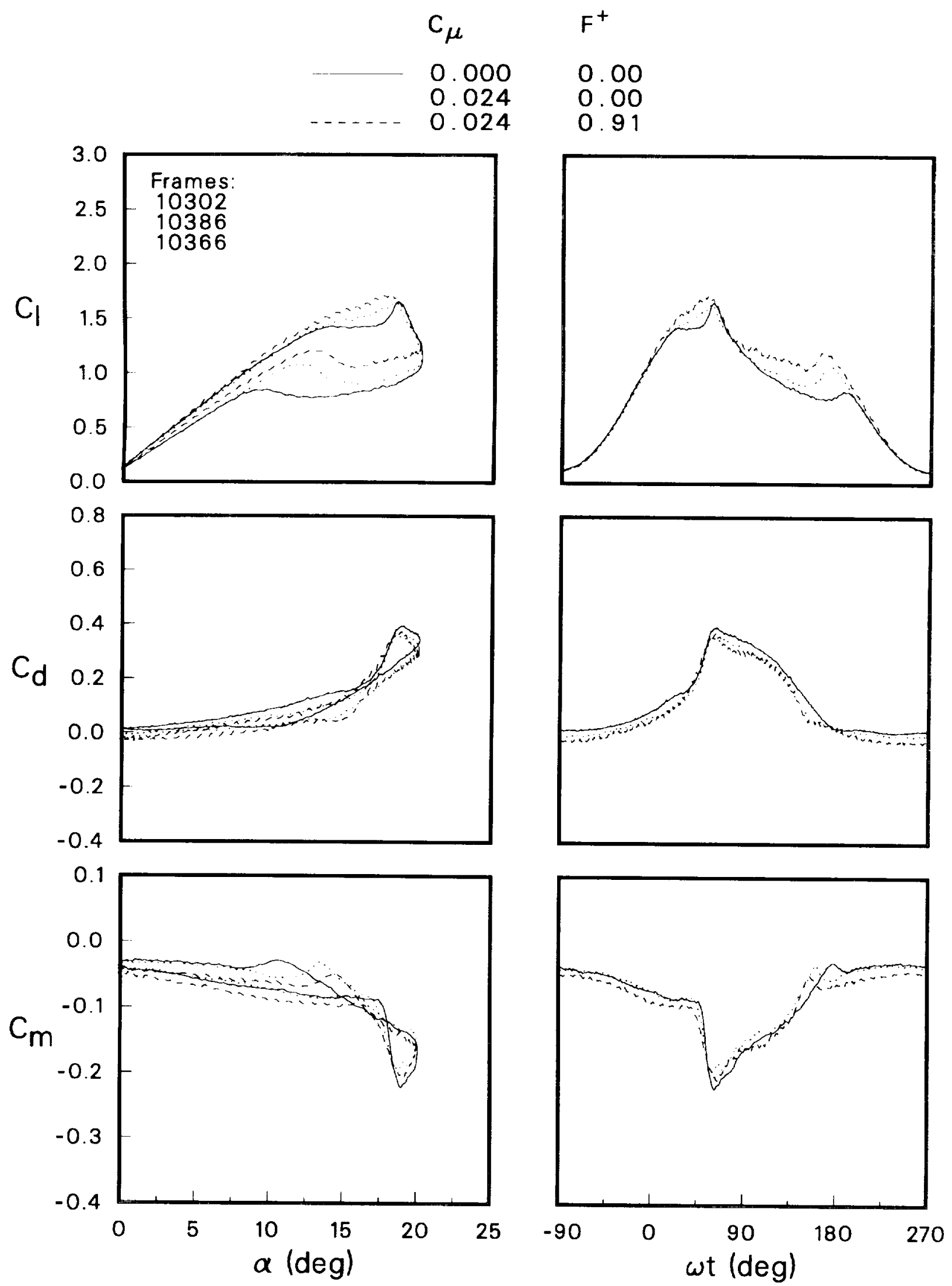

Figure 46. Effects of steady and unsteady blowing for $\alpha_{m}=10^{\circ}$ and $k=0.05$. 

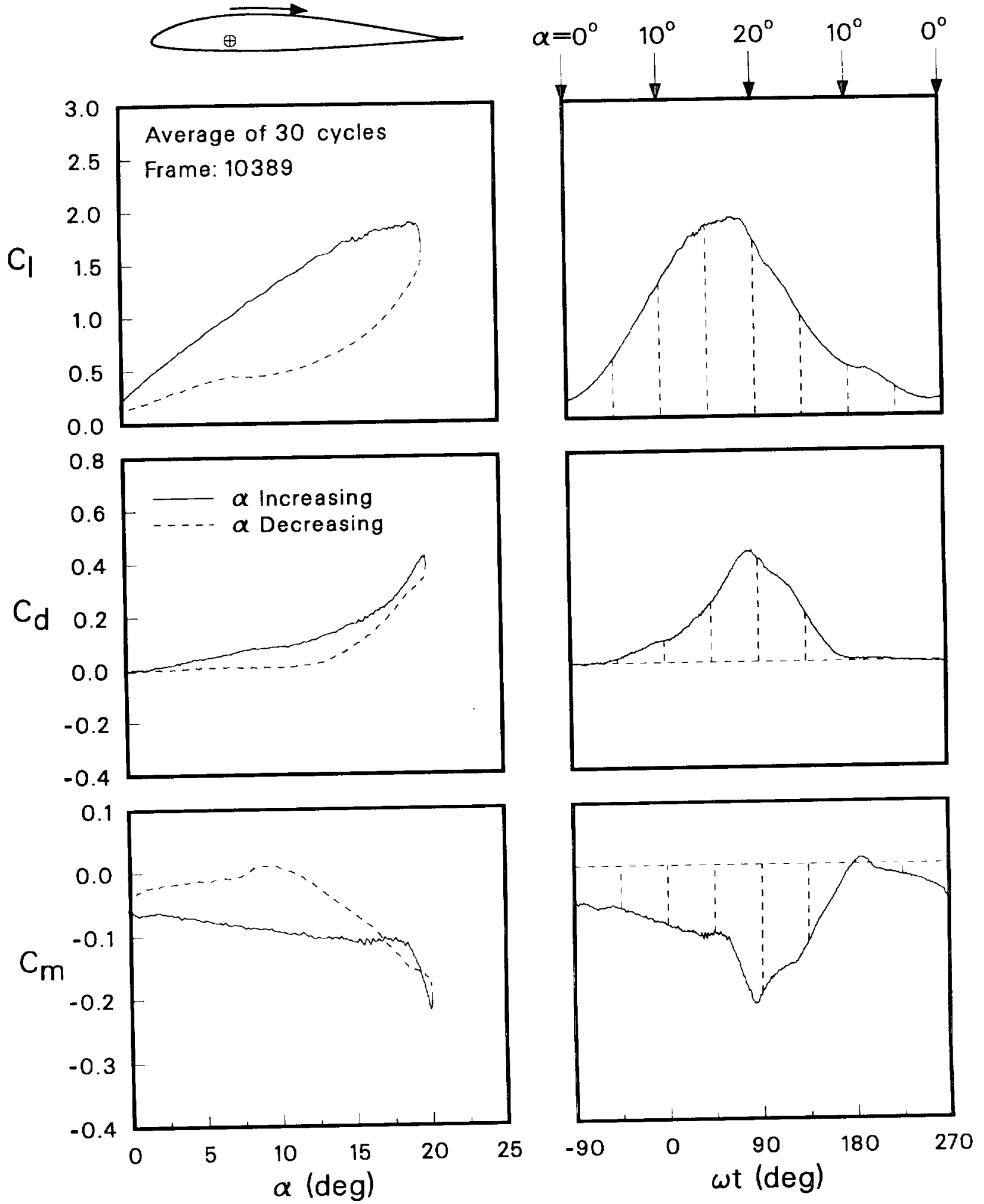

Figure 47. Unsteady loads for $\alpha_{m}=10^{\circ}$ and $k=0.15$ with steady blowing at $C_{\mu}=0.025$. 


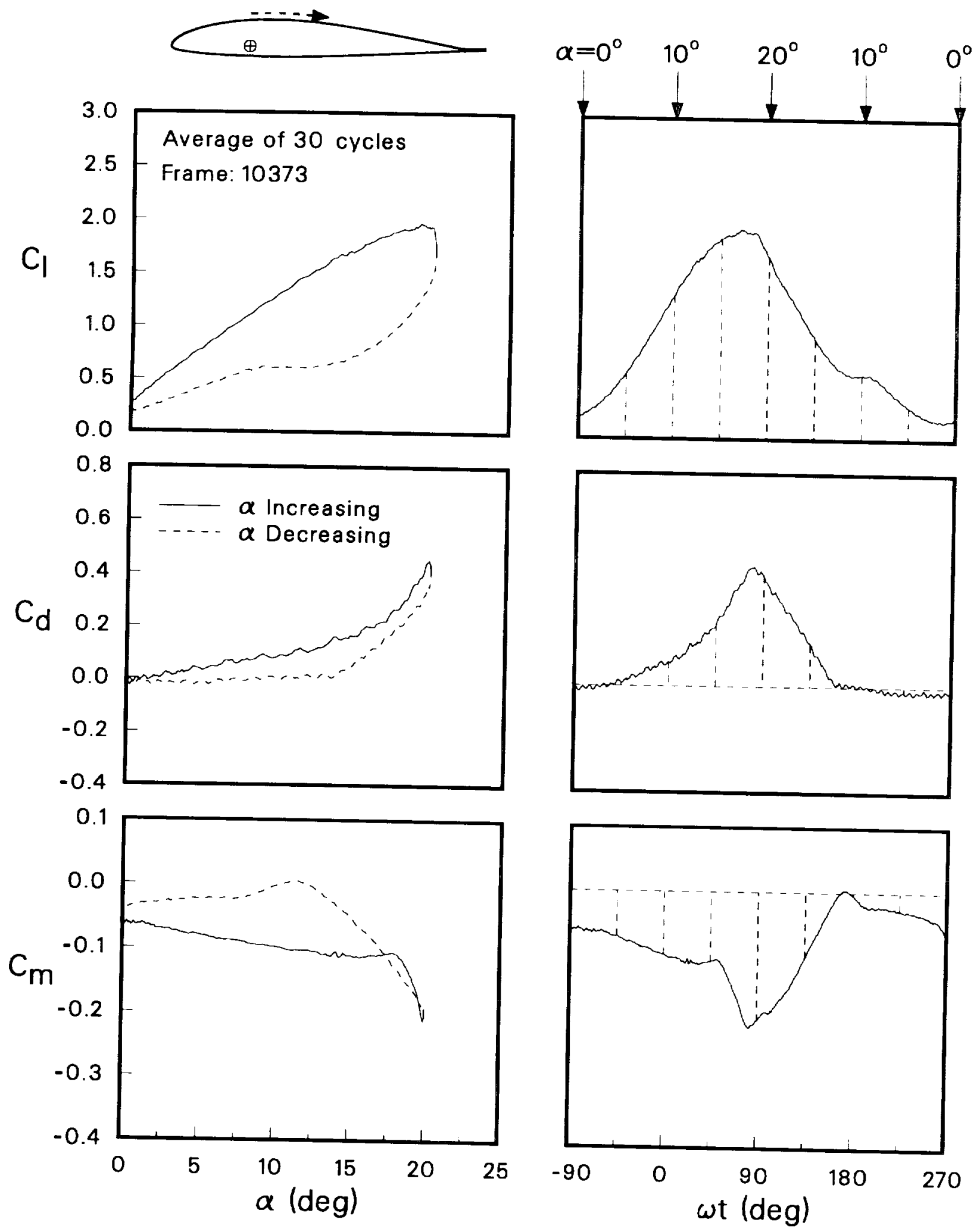

Figure 48. Unsteady loads for $\alpha_{m}=10^{\circ}$ and $k=0.15$ with unsteady blowing at $\mathrm{C}_{\mu}=0.024$ and $F^{+}=0.9$. 

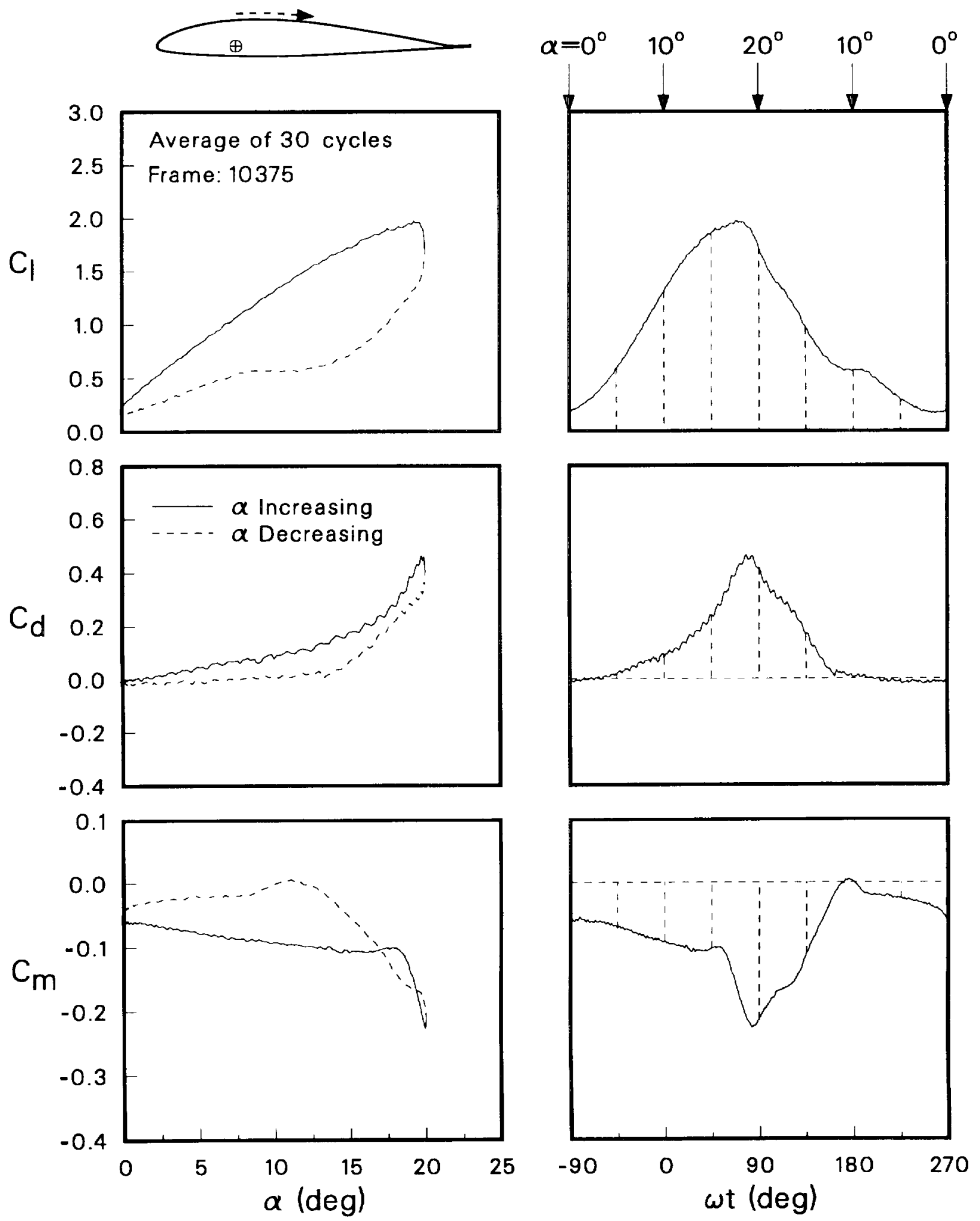

Figure 49. Unsteady loads for $\alpha_{m}=10^{\circ}$ and $k=0.15$ with unsteady blowing at $\mathrm{C}_{\mu}=0.024$ and $F^{+}=1.9$. 


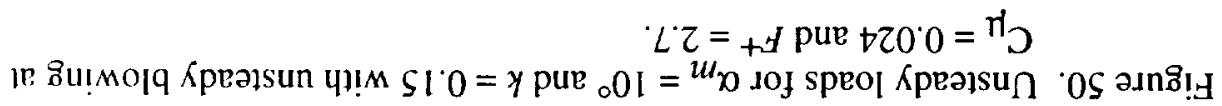

(6әр) 19
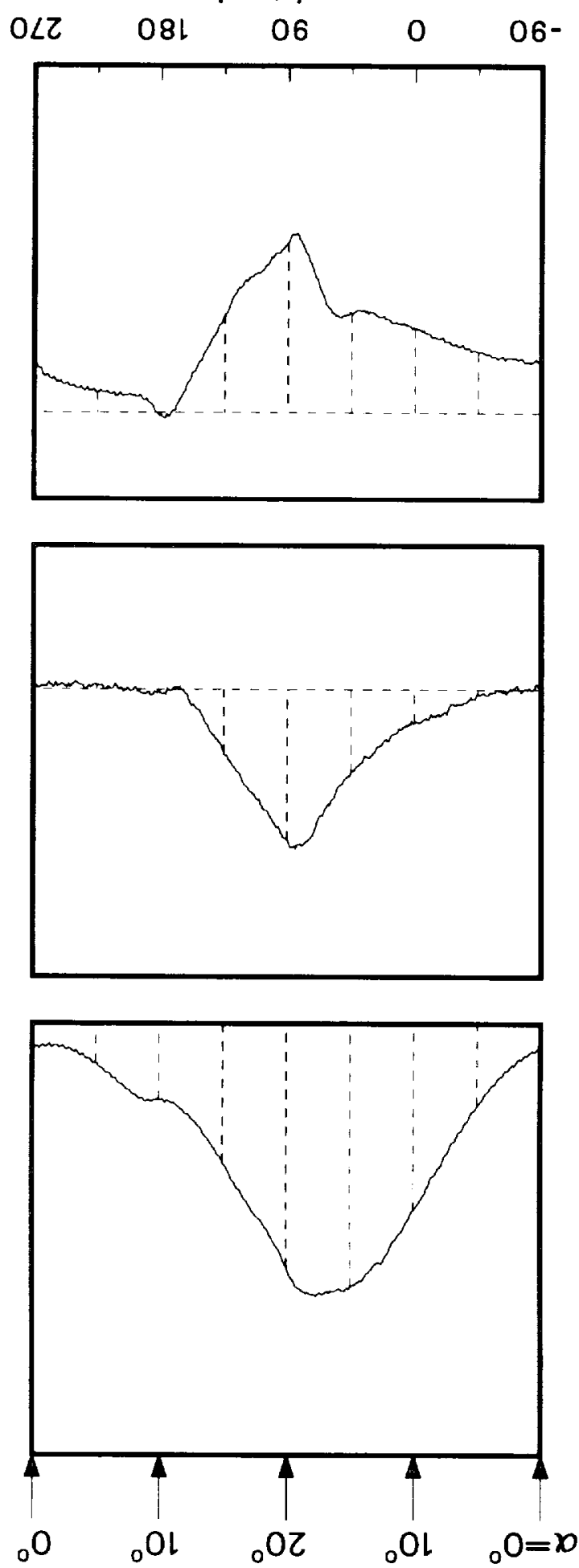

(6әр) $x$ $\begin{array}{llllll}\text { gl } & \text { or } & \text { gl } & \text { ol } & g & 0\end{array}$

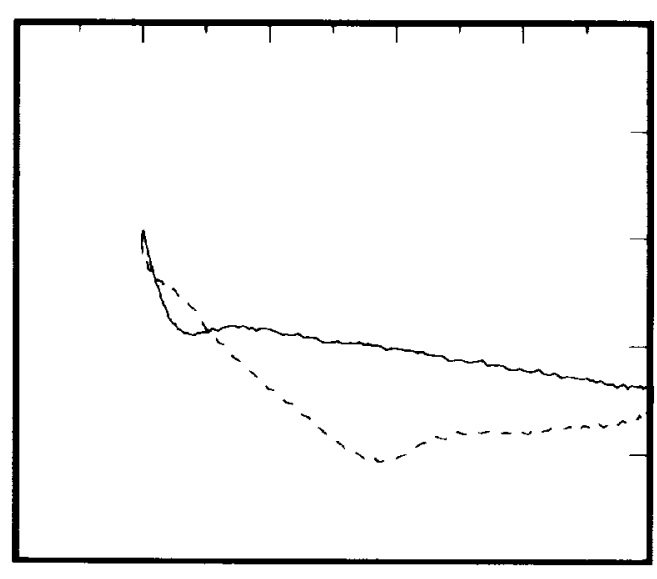

to-

$\varepsilon 0^{-}$

20-

0.0

$1 \cdot 0$

to-

Z००-

0.0

$\begin{array}{lll}2 & \mathrm{P} & 0\end{array}$

t०o

$9 \cdot 0$

Bu!seasoul $D$

8.0

0.0

g.0

0.1

s.l IJ

02

$9 \angle \varepsilon O L$ :amedy

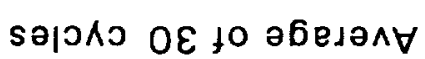

$9 \cdot 2$

$0 \cdot \varepsilon$ 


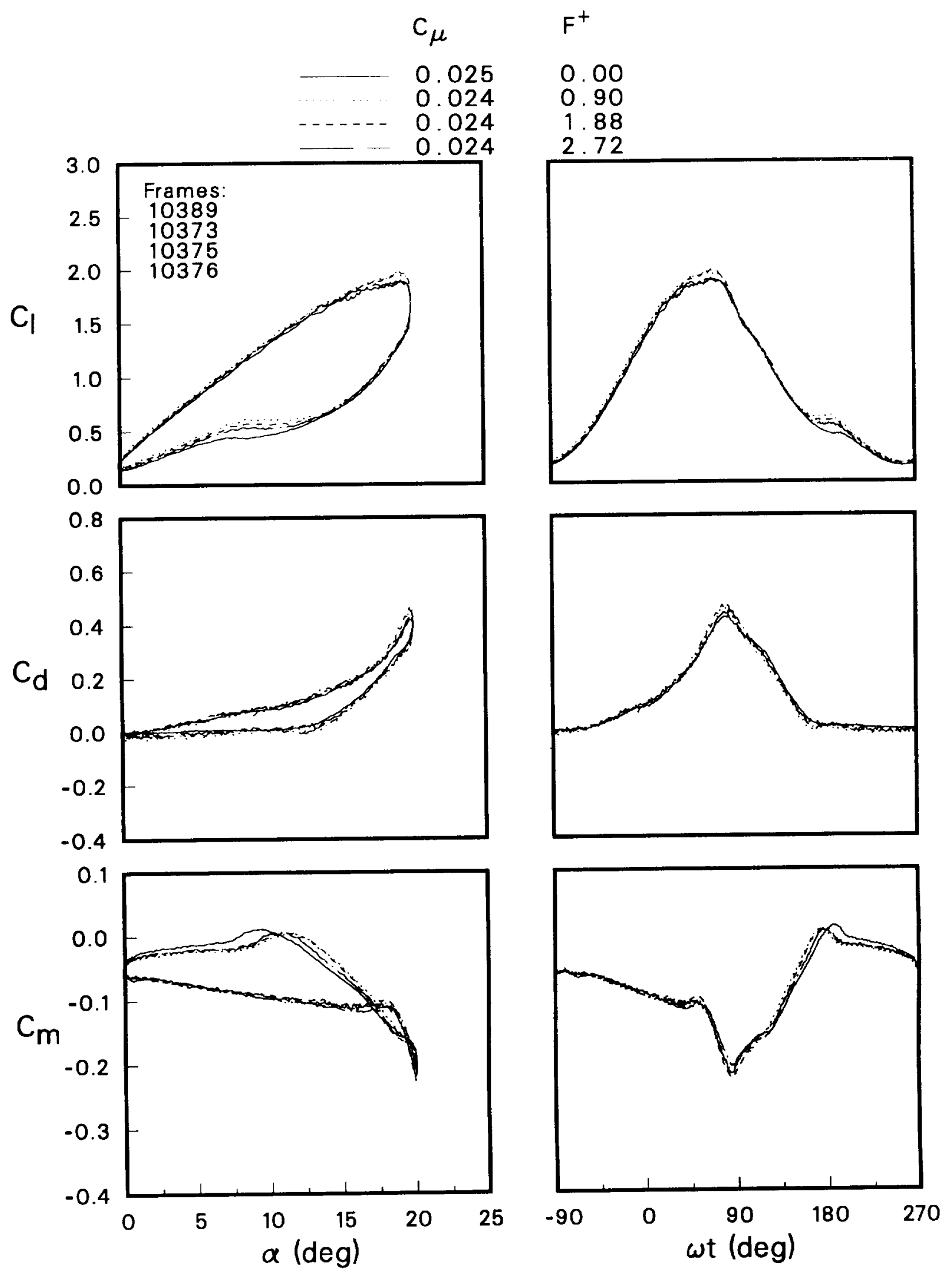

Figure 51. Effects of unsteady blowing for $\alpha_{m}=10^{\circ}$ and $k=0.15$. 


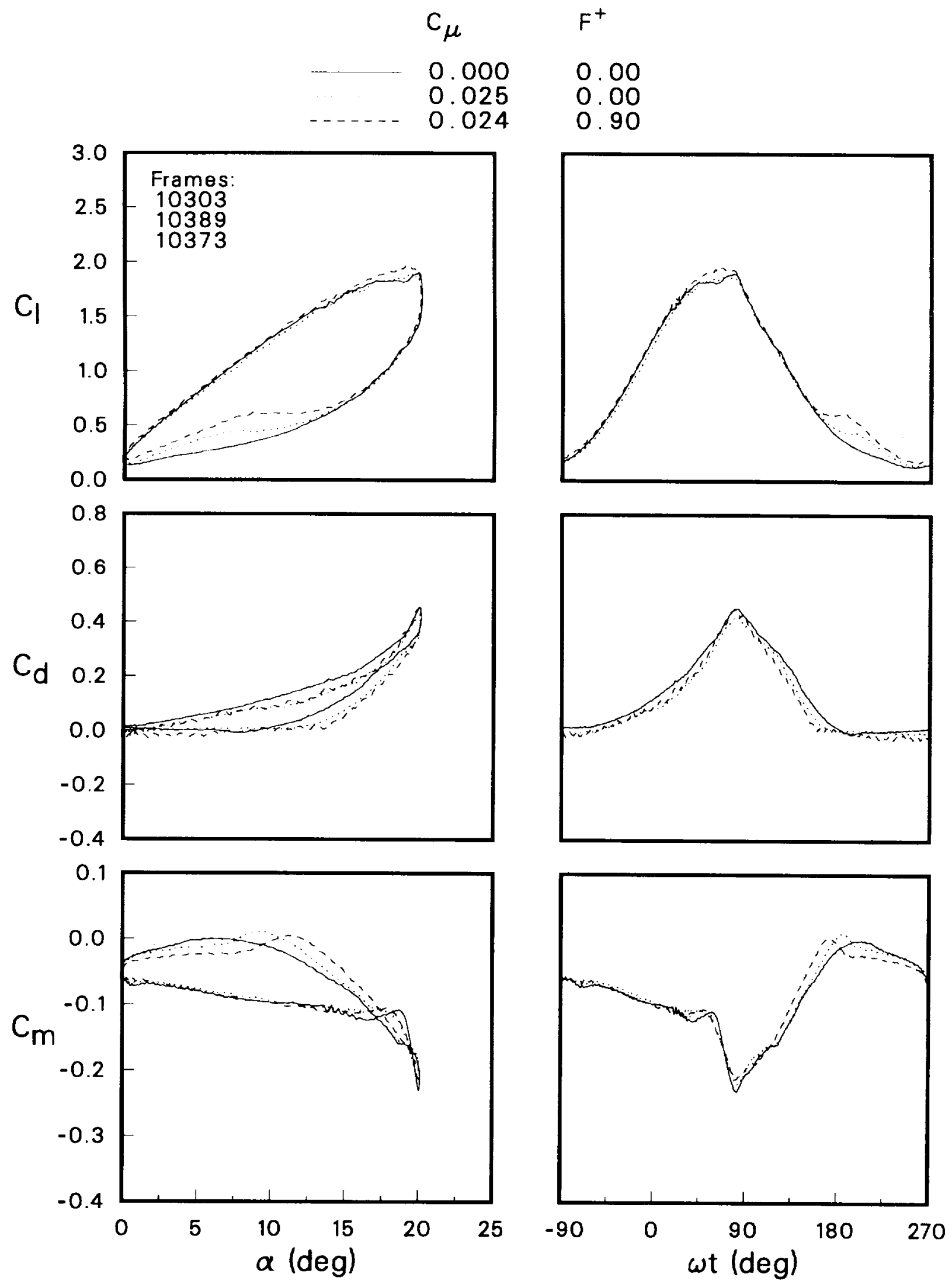

Figure 52. Effects of steady and unsteady blowing for $\alpha_{m}=10^{\circ}$ and $k=0.15$. 

Public reponting burden for this collection of information is estimated to average 1 hour per response. including the time lor reviewing instructions, searching existing data sources. gathering and maintaining the data needed, and completing and reviewing the collection of intormation. Send comments regarding this burden estimate or any other aspect of this collection of information, including suggestions for reducing this burden, to Washington Headquarters Services. Directorate for information Operations and Peports, 1215 Jefferson Davis Highway, Suite 1204, Arlington, VA 22202-4302, and to the Office of Management and Budget, Paperwork Reduction Project (0704-0188), Washington. DC 20503.

\begin{tabular}{|l|l|l|}
\hline 1. AGENCY USE ONLY (Leave b/ank) & $\begin{array}{l}\text { 2. REPORT DATE } \\
\text { February 1996 }\end{array}$ & $\begin{array}{l}\text { 3. REPORT TYPE AND DAT } \\
\text { Technical Paper }\end{array}$ \\
\hline
\end{tabular}

4. TITLE AND SUBTITLE 5. FUNDING NUMBERS

Suppression of Dynamic Stall by Steady and Pulsed Upper-Surface Blowing

6. AUTHOR(S)

$505-59-87$

D.Weaver,* K. W. McAlister, and J. Tso*

7. PERFORMING ORGANIZATION NAME(S) AND ADDRESS(ES)

8. PERFORMING ORGANIZATION REPORT NUMBER

U. S. Army Aeroflightdynamics Directorate, U.S. Army Aviation and Troop Command, Ames Research Center, Moffett Field, CA 94035-1000

A-95103

9. SPONSORING/MONITORING AGENCY NAME(S) AND ADDRESS(ES)

10. SPONSORING/MONITORING AGENCY REPORT NUMBER

National Aeronautics and Space Administration

Washington, DC 20546-0001 and U.S. Army Aviation and Troop

Command, St. Louis, MO 63120-1798

NASA TP-3600

USAATCOM TR-95-A-005

11. SUPPLEMENTARY NOTES

Point of Contact: K. W. McAlister, Ames Research Center, MS 215-1, Moffett Field, CA 94035-1000; (415) 604-5892

*California Polytechnic State University, San Luis Obispo, California

12a. DISTRIBUTION/AVAILABILITY STATEMENT

Unclassified-Unlimited

Subject Category - 02

Available from the NASA Center for AeroSpace Information,

800 Elkridge Landing Road, Linthicum Heights, MD 21090; (301) 621-0390

13. ABSTRACT (Maximum 200 words)

The Boeing-Vertol VR-7 airfoil was experimentally studied with steady and pulsed upper-surface blowing for sinusoidal pitching oscillations described by $\alpha=\alpha_{m}+10^{\circ} \sin \omega$. The tests were conducted in the U.S. Army Aeroflightdynamics Directorate's Water Tunnel at NASA Ames Research Center. The experiment was performed at a Reynolds number of 100,000 . Pitch oscillations with $\alpha_{m}=10^{\circ}$ and $15^{\circ}$ and with reduced frequencies ranging from $\mathrm{k}=0.005$ to 0.15 were examined. Blowing conditions ranged from $\mathrm{C}_{\mu}=0.03$ to 0.66 and $\mathrm{F}^{+}=0$ to 3 . Unsteady lift, drag, and pitching-moment loads were measured, and fluorescent-dye flow visualizations were obtained. Steady, upper-surface blowing was found to be capable of trapping a separation bubble near the leading edge during a portion of the airfoil's upward rotation. When this occurred, the lift was increased significantly and stall was averted. In all cases, steady blowing reduced the hysteresis amplitudes present in the loads and produced a large thrust force. The benefits of steady blowing diminished as the reduced frequency and mean angle of oscillation increased. Pulsed blowing showed only marginal benefits for the conditions tested. The greatest gains from pulsed blowing were achieved at $\mathrm{F}^{+}=0.9$.

14. SUBJECT TERMS

Airfoil stall, Dynamic stall, High-lift device, Upper-surface blowing, Stall control, Stall suppression

15. NUMBER OF PAGES

98

16. PRICE CODE

A05

\begin{tabular}{|l|l|l|l|}
\hline $\begin{array}{l}\text { 17. SECURITY CLASSIFICATION } \\
\text { OF REPORT } \\
\text { Unclassified }\end{array}$ & $\begin{array}{l}\text { 18. SECURITY CLASSIFICATION } \\
\text { OF THIS PAGE } \\
\text { Unclassified }\end{array}$ & $\begin{array}{l}\text { 19. SECURITY CLASSIFICATION } \\
\text { OF ABSTRACT }\end{array}$ & 20. LIMITATION OF ABSTRACT \\
\hline
\end{tabular}



